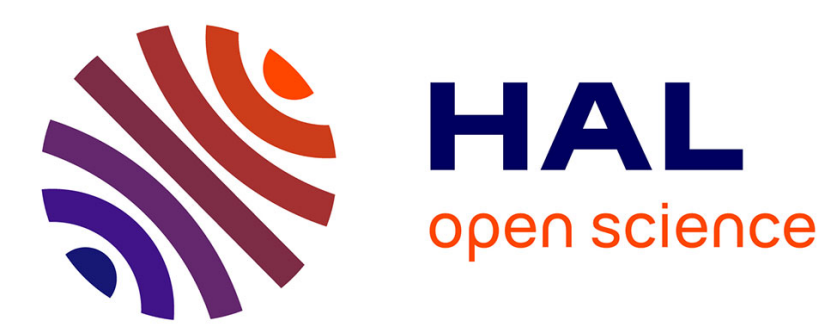

\title{
Assessment of basis sets for F12 explicitly-correlated molecular electronic-structure methods
}

Florian A. Bischoff, Sandra Wolfsegger, David P. Tew, Wim Klopper

\section{To cite this version:}

Florian A. Bischoff, Sandra Wolfsegger, David P. Tew, Wim Klopper. Assessment of basis sets for F12 explicitly-correlated molecular electronic-structure methods. Molecular Physics, 2009, 107 (08-12), pp.963-975. 10.1080/00268970802708942 . hal-00513248

\section{HAL Id: hal-00513248 \\ https://hal.science/hal-00513248}

Submitted on 1 Sep 2010

HAL is a multi-disciplinary open access archive for the deposit and dissemination of scientific research documents, whether they are published or not. The documents may come from teaching and research institutions in France or abroad, or from public or private research centers.
L'archive ouverte pluridisciplinaire HAL, est destinée au dépôt et à la diffusion de documents scientifiques de niveau recherche, publiés ou non, émanant des établissements d'enseignement et de recherche français ou étrangers, des laboratoires publics ou privés. 


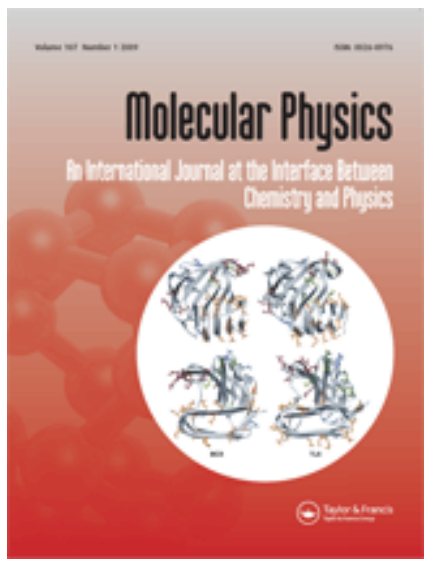

\section{Assessment of basis sets for F12 explicitly-correlated molecular electronic-structure methods}

\begin{tabular}{|c|c|}
\hline Journal: & Molecular Physics \\
\hline Manuscript ID: & TMPH-2008-0361.R1 \\
\hline Manuscript Type: & Special Issue Paper - Fritz Schaefer \\
\hline $\begin{array}{r}\text { Date Submitted by the } \\
\text { Author: }\end{array}$ & 16-Dec-2008 \\
\hline Complete List of Authors: & $\begin{array}{l}\text { Bischoff, Florian; University of Karlsruhe } \\
\text { Wolfsegger, Sandra; University of Karlsruhe } \\
\text { Tew, David; University of Karlsruhe } \\
\text { Klopper, Wim; University of Karlsruhe }\end{array}$ \\
\hline Keywords: & $\begin{array}{l}\text { Basis sets, Explicitly-correlated wave functions, F12 methods, } \\
\text { Atomisation energies }\end{array}$ \\
\hline \multicolumn{2}{|c|}{$\begin{array}{l}\text { Note: The following files were submitted by the author for peer review, but cannot be converted } \\
\text { to PDF. You must view these files (e.g. movies) online. }\end{array}$} \\
\hline $\begin{array}{l}\text { TMPH-2008-0361.tex } \\
\text { TMPH-2008-0361-SI.tex }\end{array}$ & \\
\hline
\end{tabular}

\section{s ScholarONE \\ Manuscript Central}




\title{
Assessment of basis sets for F12 explicitly-correlated molecular electronic-structure methods*
}

\author{
Florian A. Bischoff, Sandra Wolfsegger, David P. Tew and Wim Klopper ${ }^{\dagger}$ \\ Lehrstuhl für Theoretische Chemie, Institut für Physikalische Chemie, \\ Universität Karlsruhe (TH), D-76128 Karlsruhe, Germany
}

(Dated: December 16, 2008)

\begin{abstract}
One-electron basis sets for F12 explicitly-correlated molecular electronic-structure methods are assessed by analysing the accuracy of Hartree-Fock energies and valence-only second-order correlation energies of a test set of 106 small molecules containing the atoms $\mathrm{H}, \mathrm{C}, \mathrm{N}, \mathrm{O}$ and F. For these molecules, near Hartree-Fock-limit energies and benchmark second-order correlation energies (accurate to within $99.95 \%$ of the basis-set limit) are provided. Absolute energies are analysed as well as the Hartree-Fock and second-order correlation contributions to the atomisation energies of the molecules. Standard basis sets such as the Karlsruhe def2-TZVPP and def2-QZVPP sets and the augmented correlation-consistent polarised valence X-tuple zeta (aug-cc-pVXZ, X = D, T, $\mathrm{Q}, 5)$ sets are compared with the specialised cc-pVXZ-F12 (X = D, T, Q) sets that were recently optimised by Peterson and co-workers [J. Chem. Phys. 128, 084102 (2008)] for use in F12 theory. The results obtained from F12 explicitly-correlated molecular electronic-structure calculations are compared with those that are obtained by standard electronic-structure calculations followed by basis-set extrapolation based on the $\mathrm{X}^{-3}$ convergence behaviour of the aug-cc-pVXZ basis sets. The most important conclusions are that the cc-pVXZ-F12 sets are the preferred basis sets for F12 theory and that the $\mathrm{X}^{-3}$ extrapolation from the aug-cc-pVQZ and aug-cc-pV5Z is slightly more accurate than F12 theory in the cc-pVTZ-F12 basis but less accurate than F12 theory in the cc-pVQZ-F12 basis.
\end{abstract}

\footnotetext{
* Dedicated to Professor Henry F. Schaefer III on the occasion of his $65^{\text {th }}$ birthday.

$\dagger$ E-mail: klopper@chem-bio.uni-karlsruhe.de. Fax: +49-721-6083319.
} 


\section{INTRODUCTION}

F12 explicitly-correlated molecular electronic-structure methods are emerging as practical tools for computational chemistry. These methods overcome the extremely slow convergence of orbital expansions with the basis size by using additional geminal basis functions, chosen to closely approximate the form of the correlation hole at short interelectronic distances. In the early days, high-end electronic-structure calculations focused on the complete-basis-set limit, using very large one-electron basis sets. ${ }^{1}$ Examples include the calculation of the heat of formation of $\mathrm{NCO},{ }^{2}$ the calculation of barriers to linearity of small molecules such as $\mathrm{H}_{2} \mathrm{O}^{3,4}$ and $\mathrm{SiC}_{2},{ }^{5}$ the calculation of anharmonic potential energy surfaces of molecules such as $\mathrm{SiH}_{3}^{-},{ }^{5} \mathrm{BH}_{5},{ }^{6} \mathrm{NH}_{3}{ }^{7}$ and $\mathrm{H}_{3} \mathrm{O}^{+}, 8$ or the calculation of the water dimer ${ }^{9,10}$ and water. . $\mathrm{H}_{2}{ }^{11,12}$ potentials in the complete-basis-set limit of subchemical accuracy. The requirement for large basis sets in combination with geminals linear in the interelectronic distances strongly restrained applications to larger chemical systems, although at the MP2 level, systems such as [10]annulene, ${ }^{13}$ ferrocene ${ }^{14}$ and the benzene dimer ${ }^{15,16}$ could be treated.

There have been many advances in F12 theory over the last six years (see Ref. 17 for a good review) and it is now possible to use the standard orbital basis sets of quantum chemistry ${ }^{18}$ for example the def2-QZVPP basis, to obtain $\sim 99.5 \%$ of the basis set limit correlation energy for the MP2, ${ }^{19-21} \mathrm{CCSD}^{22-34}$ and CASPT2 ${ }^{35}$ methods. Indeed, MP2-R12 calculations have recently been used to reduce basis set incompleteness errors in CCSD(T) energy differences to obtain highly accurate predictions of $\pi$-stacking and hydrogen bonding interactions of the nucleobase mimic 2-pyridone with fluorobenzenes. ${ }^{36,37} \mathrm{~A}$ similar MP2F12/def2-QZVPP approach has been used to compute the heat of formation of the $\mathrm{HOSO}_{2}$ radical, an important intermediate in the atmospheric oxidation of $\mathrm{SO}_{2}$ to $\mathrm{SO}_{3}$, to an accuracy of $3 \mathrm{~kJ} / \mathrm{mol}^{38}$ Further examples include the calculation of the thermochemistry of the $\mathrm{HOSO}_{2}+\mathrm{O}_{2}$ reaction and heat of formation of the $\mathrm{HOSO}_{4}$ radical, ${ }^{39}$ calculations on rotational conformers of the alkanols n-propanol through n-pentanol, ${ }^{40}$ the computation of interactions between $\mathrm{CO}_{2}$ and N-containing organic heterocycles, ${ }^{41}$ and the computation of hydrogenation energies of benzene and naphthalene, ${ }^{42}$ for which chemical accuracy was obtained. MP2-F12/def2-QZVPP calculations have been combined with CCSD(T) calculations to construct a potential energy surface for malonaldehyde, a prototype for hydrogen transfer reactions, which was used to compute the tunnelling splitting to within $1 \mathrm{~cm}^{-1}$ 
of observation. ${ }^{43}$ F12 methods have also been successfully combined with localisation techniques to approach linear scaling, which has permitted studies of enzyme reactions in a QM/MM framework. ${ }^{44,45}$

Despite the continuing success of F12 methods, as yet fairly little is known about the relative performance of F12 calculations with the various orbital sets. Indeed, standard basis sets are far from optimal. Functions with large angular momentum quantum number and high exponents are not necessary in F12 calculations, but diffuse functions are more important than in conventional methods. ${ }^{46}$ Recently, Peterson and co-workers have constructed orbital basis sets for the atoms H, He, B-Ne, and Al-Ar, specifically optimised for F12 methods. ${ }^{47}$ They report that their cc-pVXZ-F12 basis sets provide total and relative correlation energies equivalent to that obtained using aug-cc-pV $(\mathrm{X}+1) \mathrm{Z}$ basis sets for $\mathrm{MP} 2-$ F12 calculations. However, the number of functions for each angular momentum quantum number is not the same in the cc-pVXZ-F12 and aug-cc-pVXZ sets-overall the F12 sets are substantially larger - and it is not yet clear whether these new sets represent a saving in computational effort.

In this work we provide frozen core (fc) MP2 correlation energies, converged to within 99.95\% of the basis set limit, and use them to assess the new F12 basis sets together with the correlation-consistent aug-cc-pVXZ sets of Dunning and co-workers ${ }^{48-50}$ and the Karlsruhe basis sets def2-TZVPP and def2-QZVPP. ${ }^{51}$ This very high accuracy of $99.95 \%$ is necessary to rigorously assess F12 methods. We only consider the elements H, C, N, O and F. For our assessment set, we choose the set of 105 molecules compiled by Bakowies ${ }^{52}$ to which we add $\mathrm{H}_{2}$. This set represents a wide range of bonding situations for these five elements and is intended as a general testing set for F12 methods. We have used this same set to develop explicitly-correlated model chemistries, combining F12 calculations with post-CCSD(T) correlation contributions and relativistic corrections, aiming at chemical accuracy. ${ }^{53}$ The structures for the 106 molecules have been optimised at the all-electron CCSD(T)/cc-pCVTZ level of theory and are reported in the Supplementary Information.

This article is organised as follows: In Section II we present the benchmark fc-MP2 correlation energies, giving the details how they were computed. In Section III we analyse the remaining basis set error and the errors arising from the resolution of the identity approximations intrinsic to the F12 methods and the density fitting employed in our program. In Section IV we use our benchmark data to assess the performance of F12 and standard orbital 
basis sets when used in MP2-F12 calculations for both absolute and relative energies.

\section{MP2 BASIS SET LIMIT}

Since fc-MP2-F12 calculations with quadruple- $\zeta$ quality basis sets recover $\sim 99.5 \%$ of the basis set limit correlation energy, it is necessary to compute the fc-MP2 limit to better than 99.95\% to compare the performance of different quadruple- $\zeta$ sets. In Table I we present our reference fc-MP2 basis set limit correlation energies for the 106 molecules and the four atoms $\mathrm{C}, \mathrm{N}, \mathrm{O}$ and $\mathrm{F}$ in our test set. We also report the near basis set limit HF energies obtained using our decontracted aug-cc-pV6Z basis. The correlation energies are converged to within the required $99.95 \%$. The $0.05 \%(0.05 \mathrm{~kJ} / \mathrm{mol}$ per valence electron $)$ deviation from the basis set limit contains a variety of contributions, all of which are discussed and quantified in Section III.

To achieve 99.95\% accuracy, we use the MP2-F12 method with a decontracted augcc-pV6Z orbital basis. All primitives with exponents of less than $1000 a_{0}^{-2}$ were used as decontracted basis functions and contractions involving primitives with higher exponents were retained to avoid problems in density fitting. The MP2-F12 calculations were performed using a correlation factor of six Gaussian geminals, fitted to an exponential with exponent $1.4 a_{0}^{-1}, 46,54$ and the F12 amplitudes were optimised using the orbital invariant method. ${ }^{55}$ Ansatz 2 and approximation B were used and the commutator integrals involving the kinetic energy were computed from the matrix representation of the core Hamiltonian. The auxiliary basis set for the resolution-of-the-identity (RI) approximation intrinsic to the MP2-F12 method was taken to be the union of the orbital basis and a complementary auxiliary basis (CABS), ${ }^{56}$ which was chosen to be the aug-cc-pV5Z MP2-fitting basis (aug-cc-pV5Z cbas in TURBOMOLE jargon, vide infra). ${ }^{57}$ For the open-shell calculations, the ROHF wave function was used and UMP2-F12 calculations were performed using semi-canonical orbitals.

All calculations have been performed with the ricc2 module ${ }^{58}$ of the TURBOMOLE program package, ${ }^{59}$ which uses density fitting for all four-centre two-electron integrals. ${ }^{60}$ The details of the RI-MP2 approach are found in Refs. 61 and 62 and the details of the RI-MP2F12 implementation are found in Refs. 40 and 63. The MP2-F12 equations contain the Fock matrix in the unified orbital-plus-CABS space and six kinds of four-centre two-electron inte- 
grals involving the Coulomb operator, the correlation factor, or both together. The density fitting for the Coulomb and exchange contributions to the Fock matrix (RI-JK approximation) was performed using the aug-cc-pV5Z RI-JK-fitting auxiliary basis (aug-cc-pV5Z jkbas in TURBOMOLE jargon) of Weigend ${ }^{64,65}$ and the density fitting for the remaining four-centre integrals was performed using a specially constructed auxiliary basis. Taking the aug-cc-pV6Z MP2-fitting auxiliary basis as a starting point, for each angular quantum number, additional functions were introduced with exponents bisecting those of the augcc-pV6Z basis. Furthermore, for each angular quantum number, three steep functions and three diffuse functions were added. The new functions extend the range of exponents represented in an even tempered way, using the highest, or lowest, two exponents to determine the series. In MP2-F12 theory, the density fitting basis must be suitable for both the orbital space and the CABS space. This extensive basis was thus necessary and ensured that the B matrix was positive definite for all but three molecules, difluoroacetylene (no. 36), carbon suboxide (no. 67) and dicyanoacetylene (no. 72). For these molecules the MP2-F12 energy was computed using F12 amplitudes predetermined by the coalescence conditions. ${ }^{66}$

\section{ERROR ANALYSIS}

To obtain fc-MP2-F12 correlation energies very close to the basis set limit, one must obtain convergence with respect to the orbital basis and the various auxiliary basis sets employed. In our calculations, this includes the auxiliary basis for MP2 fitting (cbas), the auxiliary basis for constructing the Fock operator ( $j k b a s)$ and the complementary auxiliary basis (cabs) for the many-electron RI. Furthermore, the errors introduced by neglecting contributions to the three- and four-electron integrals must also be controlled. In our MP2F12 implementation we neglect some terms corresponding to Fock matrix elements between occupied and CABS orbitals (i.e., we assume that the generalised Brillouin condition (GBC) holds).

For conventional calculations, the behaviour of the error with respect to the orbital and auxiliary basis sets is well known. The error with respect to orbital basis converges as $\mathrm{X}^{-3}$, where $\mathrm{X}$ is the cardinal number of the basis. The error in the energy with respect to the density fitting for the Hartree-Fock and MP2 integrals reduces quadratically with the size of the auxiliary basis sets. The situation for MP2-F12 is rather different. The convergence 
with respect to the orbital basis is predicted to reduce as $\mathrm{X}^{-7}$, but the effect on the energy due to the density fitting and CABS-RI approximations is less well defined than in the conventional case because of the complexity of the equations. Nonetheless, we expect that comparing energies computed with two auxiliary basis sets representing cardinal numbers $\mathrm{X}$ and $\mathrm{X}-1$ will give a conservative estimation of the remaining error associated with using the auxiliary basis $\mathrm{X}$. This is the approach we adopt to assess the errors due to the auxiliary basis sets. To avoid problems with numerical stability, the fixed-amplitude method is used for these comparisons. Our investigations show that this restriction effects the reported errors only in the third significant figure.

To facilitate a fair and transparent comparison of the errors arising due to the various finite basis sets and approximations, we report errors per valence electron $\Delta=\left(E_{\text {app. }}-\right.$ $\left.E_{\text {ref. }}\right) / N_{\text {val. }}$ for the molecules or reactions under consideration, in $\mathrm{kJ} / \mathrm{mol}$. This measure is also used for the error in the HF energy, which is reasonable because the error due to the core electrons is negligible in our orbital basis. In the discussion, we also refer to percentage errors, defined as $100\left(E_{\text {app. }}-E_{\text {ref. }}\right) / E_{\text {ref. }}$. We report a statistical analysis of the errors in terms of the mean error $\bar{\Delta}$, the standard deviation $\sigma$, the mean absolute error $\Delta_{\text {abs. }}$, the root mean square error $\Delta_{\text {rms }}$, and maximum error $\Delta_{\text {max. }}$ :

$$
\begin{aligned}
\bar{\Delta} & =\frac{1}{N} \sum_{i=1}^{N} \Delta_{i} \\
\sigma^{2} & =\frac{1}{N} \sum_{i=1}^{N}\left(\Delta_{i}-\bar{\Delta}\right)^{2}, \\
\Delta_{\text {abs. }} & =\frac{1}{N} \sum_{i=1}^{N}\left|\Delta_{i}\right| \\
\Delta_{\text {rms }}^{2} & =\frac{1}{N} \sum_{i=1}^{N} \Delta_{i}^{2} .
\end{aligned}
$$

The dominant contribution to the deviation from the true basis set limit is the incompleteness of the orbital basis. Past experience, as well as our current calculations, show that the MP2-F12 energies computed with approximation A (cf. Ref. 67 for the definition of the approximations A and B) converge from above, whereas the energies computed using approximation B exhibit the usual convergence from below. To estimate the remaining basis set error, we assume that the MP2-F12/2A correlation energy, computed using the decontracted aug-cc-pV6Z basis, represents an upper bound. A conservative error estimate is thus 
obtained from a statistical analysis of the deviation of the MP2-F12/2B energies from the MP2-F12/2A energies. The resulting statistical measures of the error per valence electron due to the incompleteness of the orbital basis are reported in Table II. The mean error and standard deviation of the orbital-basis error distribution are $0.042 \mathrm{~kJ} / \mathrm{mol}(0.041 \%)$ and $0.010 \mathrm{~kJ} / \mathrm{mol}(0.010 \%)$ respectively, and we therefore conclude that the fc-MP2 correlation energies are converged to within $0.05 \mathrm{~kJ} / \mathrm{mol}(0.05 \%)$ of the basis set limit, per valence electron. As an aside, we mention that further decontraction of the aug-cc-pV6Z basis only lowered the correlation energy by $0.003 \mathrm{~kJ} / \mathrm{mol}$ per heavy atom, well below the basis set error due to higher angular momentum functions. The effect on the HF energy was even smaller.

The basis set limit correlation energies of three of the molecules in our set were computed using the fixed-amplitude method. For the remaining 103 molecules in our set, we were able to compare the MP2-F12 energies computed using fixed or variationally optimised amplitudes. The fixed-amplitude method systematically returns $\sim 0.004 \mathrm{~kJ} / \mathrm{mol}$ less correlation energy per valence electron than when the amplitude are optimised. This error is of little consequence compared to the remaining $0.05 \%$ orbital basis-set-incompleteness error and we consider that we are justified in using the energies from the fixed-amplitude method as the reference basis set limit correlation energies for the three molecules difluoroacetylene (no. 36), carbon suboxide (no. 67) and dicyanoacetylene (no. 72).

In our MP2-F12 implementation, the exchange matrix elements are used to evaluate the integrals over $\left[K_{1}+K_{2}, f_{12}\right]$ and the Fock matrix elements are used to evaluate the integrals over $\left[Q_{12}, F_{1}+F_{2}\right]$ and also the coupling terms between the conventional and F12 amplitudes. $F$ and $K$ are the Fock and exchange operators respectively, $f_{12}$ is the correlation factor and $Q_{12}$ is the strong-orthogonality operator. All of these are comparatively small contributions and the error in the correlation energy arising from the RI-JK density fitting is thus expected to be tiny. To quantify this error, we have performed calculations using the aug-cc-pV5Z and aug-cc-pVQZ RI-JK auxiliary basis sets, keeping all other basis sets as those detailed in Section II. An estimate of the density fitting error is obtained for each molecule as the difference between the MP2-F12/2B-fixed energies computed using these two RI-JK auxiliary basis sets. From the row RI-JK in Table II we see that the aug-ccpVQZ auxiliary basis gives correlation energies systematically smaller in magnitude than the aug-cc-pV5Z basis, by $0.0001 \mathrm{~kJ} / \mathrm{mol}$ per valence electron. The standard deviation of 
the error distribution is $0.00001 \mathrm{~kJ} / \mathrm{mol}$ and it is clear that the error in the RI-JK basis has essentially zero contribution to the deviation from the true basis set limit.

To estimate the error in the correlation energy arising from the density fitting of the MP2type four-centre two-electron integrals, we have performed calculations using two densityfitting basis sets. We have used the specially constructed auxiliary set described in Section II and the aug-cc-pV6Z MP2-fitting auxiliary basis set. In Table II we present the statistical errors for the differences in the conventional fc-MP2 energies (denoted RI-MP2), in the F12 contribution to the fc-MP2-F12-fixed energies (denoted RI-F12) and the total fc-MP2F12-fixed correlation energy (denoted MP2-Tot.). It is remarkable that the density fitting errors in the conventional and F12 energy contributions consistently almost entirely cancel each other. The mean density-fitting error is $-0.0001 \mathrm{~kJ} / \mathrm{mol}$ per valence electron and the standard deviation is $0.0001 \mathrm{~kJ} / \mathrm{mol}$. Just as for the RI-JK error, the density fitting error is completely negligible in our calculations and even acts on average to cancel the RI-JK error.

The basis for the RI intrinsic to F12 theory is the union of the orbital and complementary auxiliary basis sets. This basis is used in many places to reduce three- and four-electron integrals, involving several combinations of operators, to two-electron integrals. It is also used for the matrix representation of the core Hamiltonian operator. We have performed calculations using the aug-cc-pV5Z and aug-cc-pVQZ MP2-fitting basis sets as CABS to estimate the error due to the incomplete basis for this RI error. The mean error is -0.0024 $\mathrm{kJ} / \mathrm{mol}$ per valence electron, which represents a systematic shift to correlation energies that are too large in magnitude. The standard deviation is $0.0005 \mathrm{~kJ} / \mathrm{mol}$. Although this error is much larger than that of density fitting, it is still an order of magnitude smaller than the error due to the incomplete orbital basis.

Two further sources of error remain to be discussed. These are both related to the lack of convergence of the Hartree-Fock reference with respect to the orbital basis. The first is the GBC assumption, used in the evaluation of the B matrix in MP2-F12 theory. The second is the direct impact of using unconverged Hartree-Fock orbitals and orbital energies in the MP2 expressions. The importance of the Fock matrix elements between occupied and CABS orbitals may be assessed by computing the contribution to the second-order energy when these terms are treated as a perturbation and result in single excitations into orbitals in the CABS space. ${ }^{6}$ The mean energy of this CABS singles correction is $-0.0026 \mathrm{~kJ} / \mathrm{mol}$ per valence electron, with a standard deviation of $0.0006 \mathrm{~kJ} / \mathrm{mol}$. Although this CABS-singles 
perturbation energy is not a direct measure of either of the two aforementioned errors, it is certainly an indication that these errors may safely be neglected in the decontracted aug-ccpV6Z basis, particularly because the effect of unconverged orbitals on the correlation energy has previously been observed to be two orders of magnitude smaller than the effect on the Hartree-Fock energy.

An estimate of the overall deviation of our reference fc-MP2 correlation energies from the basis-set limit is obtained by Gaussian error propagation of the Orb., CABS, RI-Tot. and RIJK errors. The resulting mean error is $0.040 \mathrm{~kJ} / \mathrm{mol}$ per valence electron and the standard deviation is $0.01 \mathrm{~kJ} / \mathrm{mol}$. This error is completely dominated by the incompleteness of the orbital basis and our conservative estimates give a $95 \%$ confidence interval with a maximum error of $0.05 \mathrm{~kJ} / \mathrm{mol}$ per valence electron $(0.06 \%)$. For the Hartree-Fock basis set limit reference value we use the values computed with the decontracted aug-cc-pV6Z basis. No RI approximations were employed for the Hartree-Fock energy. Since the change in energy upon further decontraction or upon including the CABS singles correction was below 0.005 $\mathrm{kJ} / \mathrm{mol}$ per valence electron on average, the error in the total fc-MP2 electronic energies may also be taken to be $0.05 \mathrm{~kJ} / \mathrm{mol}$ per valence electron.

\section{BASIS SET ASSESSMENT}

\section{A. Absolute Hartree-Fock and correlation energies}

Having determined the fc-MP2 basis set limit to $99.95 \%$ accuracy, we may now proceed to assess the various orbital basis sets for use with the MP2-F12 method. The predominant basis sets in current usage are the aug-cc-pVXZ sets of Dunning and co-workers, the Karlsruhe def2-TZVPP and def2-QZVPP sets and the newly developed cc-pVXZ-F12 sets of Peterson and co-workers. We have computed the fc-MP2-F12/2B energies using each of these basis sets, always in combination with all of the large auxiliary basis sets described in Section II. We used an exponent of $1.4 a_{0}^{-1}$ for the Slater-type correlation factor for all calculations except for the F12 basis sets, where we used the recommended values of 0.9 , 1.0 and $1.1 a_{0}^{-1}$ for $\mathrm{X}=\mathrm{D}, \mathrm{T}$ and $\mathrm{Q}$, respectively. Both the Hartree-Fock and correlation contributions are of interest when assessing the basis sets.

The statistical measures for the deviation of the computed Hartree-Fock energies from 
the reference values are presented in Table III. In Figure 1 we plot the mean basis-set errors (in $\mathrm{kJ} / \mathrm{mol}$ ) against the number of functions in the basis set for a first-row main-group element (C, N, O or F). Here and in all subsequent plots of this type, the standard deviation is indicated as an error bar. We see that the def2-TZVPP and def2-QZVPP basis sets are very well optimised with respect to the Hartree-Fock limit, whereas the aug-cc-pVDZ and cc-pVDZ-F12 basis sets have comparably large errors and error bars. The performance of the cc-pVTZ-F12 and cc-pVQZ-F12 basis sets is very convincing, however. Their errors are always found below the aug-cc-pVXZ curve, not only as a function of the cardinal number but also as a function of the number of basis functions. In MP2-F12 calculations the error in the HF energy can be significantly reduced by including a perturbative correction from single excitations into the CABS basis. In Table IV we present the error statistics for the corrected HF energies. This correction greatly improves the Hartree-Fock energies, reducing the error by a factor of roughly 4,3 and 2 for basis sets with cardinal numbers $\mathrm{X}=2,3$ and 4 , respectively. We note that although we have used a very large CABS basis in our calculations, the convergence of the singles correction with the CABS basis is rapid and similar values are expected when smaller CABS basis sets are used.

The corresponding error distributions for the deviation of the correlation energies from the reference basis-set limit values are reported in Table V and depicted in Figure 2. The analogous values computed using F12 amplitudes determined using the cusp conditions rather than variational optimisation are presented in the Supporting Information. There is a clear improvement in the correlation energies when progressing from the Karlsruhe sets, to the Dunning sets, to the F12 sets of Peterson. This trend is also shown clearly in Figure 3, where the mean basis set errors $(\mathrm{kJ} / \mathrm{mol})$ are plotted as a function of the number of basis functions of a first-row main-group element, as before. We see that with respect to the size of the basis, the Karlsruhe and Dunning sets lie on essentially the same curve, but the specially optimised F12 sets are a definite improvement. Although the cc-pVDZ-F12 and def2-TZVPP sets are approximately the same size, the F12 set has no $f$ functions, replacing them with diffuse $s$ and $p$ functions, which are more important for F12 calculations.

Figure 2 also contains the error distributions for the deviation of the correlation energies that are obtained by means of the $\mathrm{X}^{-3}$ extrapolation scheme of Helgaker and-coworkers. ${ }^{69}$ The mean errors in the MP2 correlation energy are 0.64 and $0.15 \mathrm{~kJ} / \mathrm{mol}$ per valence electron after extrapolating from the aug-cc-pVTZ/aug-cc-pVQZ - denoted as aug-(TQ) - or from 
the aug-cc-pVQZ/aug-cc-pV5Z results, respectively (Table V). The aug-(Q5) extrapolation is capable of recovering ca. $99.86 \%$ of the correlation energy on average with a standard deviation of only $0.04 \%(0.05 \mathrm{~kJ} / \mathrm{mol}$ per valence electron $)$. This is slightly better than the accuracy of F12/cc-pVTZ-F12 values $(0.26 \mathrm{~kJ} / \mathrm{mol}, 99.75 \%)$ but not as good as F12/ccpVQZ-F12 (0.07 kJ/mol, 99.94\%). We furthermore note that the aug-(TQ) and F12/ccpVDZ-F12 levels are of comparable accuracy.

The error distributions for the deviation of the total MP2 energy (Hartree-Fock plus correlation plus CABS singles) are reported in Table VI. We find that the most accurate results are obtained at the cc-pVQZ-F12 and aug-cc-pV5Z levels, with mean errors per valence electron of 0.10 and $0.12 \mathrm{~kJ} / \mathrm{mol}$, respectively. The mean errors are slightly larger in the aug-cc-pVQZ, cc-pVTZ-F12 and def2-QZVPP basis sets $(0.39,0.48$ and $0.51 \mathrm{~kJ} / \mathrm{mol})$. Although these three basis sets perform similarly well, the aug-cc-pVQZ basis is significantly larger than the def2-QZVPP and cc-pVTZ-F12 basis sets, which are almost equivalent in size and accuracy. We note, however, that the error in the def2-QZVPP basis is dominated by the correlation contribution, whereas the error in the cc-pVTZ-F12 basis is evenly distributed between Hartree-Fock and correlation.

\section{B. Contributions to atomisation energies}

It is important to assess the performance of the basis sets not only for total energies but also for relative energies. Using the fc-MP2-F12 method with the Peterson, Dunning and Karlsruhe basis sets, we have computed atomisation energies for the 106 molecules. Table VII contains the statistical measures for the error per valence electron $(\mathrm{kJ} / \mathrm{mol})$ in the correlation contribution to the atomisation energies and we have also included the values obtained from extrapolating conventional fc-MP2 energies. The error distributions are displayed graphically in Figure 4, and in Figure 5 the mean errors are plotted against the size of the basis. The corresponding table and figure for the Hartree-Fock (plus CABS singles) contribution are given in the Supporting Information.

Figure 4 shows that the results converge smoothly to the basis-set limits with the augcc-pVXZ and cc-pVXZ-F12 series, in terms of both mean errors and standard deviations. The error distributions for the smallest sets of the series (cc-pVDZ-F12, aug-cc-pVDZ and def2-TZVPP) are relatively broad, with standard deviations of $0.31,0.34$ and $0.17 \mathrm{~kJ} / \mathrm{mol}$ 
per valence electron, respectively. Among these sets, the def-TZVPP basis gives the smallest mean-absolute errors $(0.17 \mathrm{~kJ} / \mathrm{mol})$. The results obtained from the cc-pVTZ-F12, aug-ccpVQZ and def2-QZVPP basis sets are much better than from the smaller sets, with RMS deviations of $0.08,0.12$ and $0.12 \mathrm{~kJ} / \mathrm{mol}$, respectively. But note that the cc-pVTZ-F12 and def2-QZVPP basis sets are similar in size, whereas the aug-cc-pVQZ is significantly larger (Figure 5). The F12 basis sets of Peterson are a significant improvement over the Dunning sets, but the def2-TZVPP set is preferred over the cc-pVDZ-F12 set. We also note that the cc-pVTZ-F12 basis gives more accurate results than the (TQ) extrapolation, and similar accuracy to the (Q5) extrapolation.

In Table VIII we present the statistical errors for the sum of the fc-MP2 correlation energy plus Hartree-Fock contribution to the atomisation energy, including the correction obtained from the CABS single excitations (see also Figure 6). Such a sum corresponds to a realistic calculation of a relative energy such as an activation energy or heat of formation. We find that all F12 calculations studied in the present work yield atomisation energies accurate to within $\pm 0.5 \mathrm{~kJ} / \mathrm{mol}$ standard deviation, per valence electron (equivalent to an RMS error of $0.2 \%$ in the atomisation energies). In the cc-pVTZ-F12 and cc-pVQZ-F12 basis sets, the accuracy is better than $\pm 0.05 \mathrm{~kJ} / \mathrm{mol}$. When comparing the performance of the various basis sets, we note that the Hartree-Fock contribution converges from below and the correlation contribution from above. For the double-zeta sets, the error is dominated by that of the Hartree-Fock contribution, even though the CABS singles correction is included, but for the def2-QZVPP, aug-cc-pVQZ and aug-cc-pV5Z basis sets, the correlation error dominates. For the remaining sets, def2-TZVPP, cc-pVTZ-F12 and cc-pVQZ-F12, the mean Hartree-Fock and correlation errors are well balanced and also act to cancel each other. The cc-pVXZF12 results converge very smoothly to the reference values, and the standard deviations in these basis sets are significantly smaller than in the other sets. The only exception is the double-zeta set, where the def2-TZVPP set is preferred.

\section{CONCLUSIONS}

One-electron basis sets for F12 explicitly-correlated molecular electronic-structure methods have been assessed by analysing the accuracy of Hartree-Fock energies and valence-only second-order correlation energies (fc-MP2) of a test set of 106 small molecules containing 


\section{Acknowledgments}

This research has been supported by the Deutsche Forschungsgemeinschaft (DFG) in the framework of the Schwerpunktprogramm "First Principles Methods" (SPP 1145, grant No. KL 721/2-3). F. A. B. gratefully acknowledges support by the Fonds der Chemischen Industrie through a Chemiefonds stipend. D. P. T. gratefully acknowledges support by the DFG through grant No. TE 644/1-1.

1 E. F. Valeev, W. D. Allen, R. Hernandez, C. D. Sherrill, and H. F. Schaefer III, J. Chem. Phys. 118, $8594(2003)$.

2 M. S. Schuurman, S. R. Muir, W. D. Allen, and H. F. Schaefer III, J. Chem. Phys. 120, 11586 (2004).

3 G. Tarczay, A. G. Császár, W. Klopper, V. Szalay, W. D. Allen, and H. F. Schaefer III, J. Chem. Phys. 110, 11971 (1999). 
4 E. F. Valeev, W. D. Allen, H. F. Schaefer III, and A. G. Császár, J. Chem. Phys. 114, 2875 (2001).

5 J. P. Kenny, W. D. Allen, and H. F. Schaefer III, J. Chem. Phys. 118, 7353 (2003).

6 M. S. Schuurman, W. D. Allen, P. von Ragué Schleyer, and H. F. Schaefer III, J. Chem. Phys. 122, $104302(2005)$.

7 T. Rajamäki, M. Kállay, J. Noga, P. Valiron, and L. Halonen, Mol. Phys. 102, 21 (2004).

8 T. Rajamäki, J. Noga, P. Valiron, and L. Halonen, Mol. Phys. 102, 2259 (2004).

9 W. Klopper, J. G. C. M. van Duijneveldt-van de Rijdt, and F. B. van Duijneveldt, Phys. Chem. Chem. Phys. 2, 2227 (2000).

10 G. S. Tschumper, M. L. Leininger, B. C. Hoffman, E. F. Valeev, H. F. Schaefer III, and M. Quack, J. Chem. Phys. 116, 690 (2002).

11 A. Faure, P. Valiron, M. Wernli, L. Wiesenfeld, C. Rist, J. Noga, and J. Tennyson, J. Chem. Phys. 122, 221102 (2005).

12 P. Valiron, M. Wernli, A. Faure, L. Wiesenfeld, C. Rist, S. Kedžuch, and J. Noga, J. Chem. Phys. 129, 134306 (2008).

13 H. M. Sulzbach, H. F. Schaefer III, W. Klopper, and H. P. Lüthi, J. Am. Chem. Soc. 118, 3519 (1996).

14 W. Klopper and H. P. Lüthi, Chem. Phys. Lett. 262, 546 (1996).

15 M. O. Sinnokrot, E. F. Valeev, and C. D. Sherrill, J. Am. Chem. Soc. 124, 10887 (2002).

16 M. O. Sinnokrot and C. D. Sherrill, J. Phys. Chem. A. 110, 10656 (2006).

17 W. Klopper, F. R. Manby, S. Ten-No, and E. F. Valeev, Int. Rev. Phys. Chem. 25, 427 (2006).

18 W. Klopper and C. C. M. Samson, J. Chem. Phys. 116, 6397 (2002).

19 H.-J. Werner, T. B. Adler, and F. R. Manby, J. Chem. Phys. 126, 164102 (2007).

20 S. Ten-no, J. Chem. Phys. 126, 014108 (2007).

21 D. Yamaki, H. Koch, and S. Ten-No, J. Chem. Phys. 127, 144104 (2007).

22 D. P. Tew, W. Klopper, C. Neiss, and C. Hättig, Phys. Chem. Chem. Phys. 9, 1921 (2007).

23 D. P. Tew, W. Klopper, C. Neiss, and C. Hättig, Phys. Chem. Chem. Phys. 10, 6325 (2008), erratum.

24 T. B. Adler, G. Knizia, and H.-J. Werner, J. Chem. Phys. 127, 221106 (2007).

25 G. Knizia, T. B. Adler, and H.-J. Werner, Simplied CCSD(T)-F12 methods: Theory and benchmarks, J. Chem. Phys. (in press). 
26 D. P. Tew, W. Klopper, C. Neiss, and C. Hättig, Chem. Phys. Lett. 452, 326 (2008).

27 E. F. Valeev, Phys. Chem. Chem. Phys. 10, 106 (2008).

28 T. Shiozaki, M. Kamiya, S. Hirata, and E. F. Valeev, Phys. Chem. Chem. Phys. 10, 3358 (2008).

29 M. Torheyden and E. F. Valeev, Phys. Chem. Chem. Phys. 10, 3410 (2008).

30 E. F. Valeev and T. D. Crawford, J. Chem. Phys. 128, 244113 (2008).

31 T. Shiozaki, M. Kamiya, S. Hirata, and E. F. Valeev, J. Chem. Phys. 129, 071101 (2008).

32 J. Noga, , S. Kedžuch, J. Šimunek, and S. Ten-no, J. Chem. Phys. 128, 174103 (2008).

33 D. Bokhan, S. Ten-no, and J. Noga, Phys. Chem. Chem. Phys. 10, 3320 (2008).

34 A. Köhn, G. W. Richings, and D. P. Tew, J. Chem. Phys. 129, 201103 (2008).

35 S. Ten-no, Chem. Phys. Lett. 447, 175 (2007).

36 R. Leist, J. A. Frey, P. Ottiger, H.-M. Frey, S. Leutwyler, R. A. Bachorz, and W. Klopper, Angew. Chem. Int. Ed. 46, 7449 (2007).

37 R. A. Bachorz, F. A. Bischoff, S. Höfener, W. Klopper, P. Ottiger, R. Leist, J. A. Frey, and S. Leutwyler, Phys. Chem. Chem. Phys. 10, 2758 (2008).

38 W. Klopper, D. P. Tew, N. González-García, and M. Olzmann, J. Chem. Phys. 129, 114308 (2008).

39 N. González-García, W. Klopper, and M. Olzmann, Thermochemistry of the $\mathrm{HOSO}_{4}+\mathrm{O}_{2}$ association reaction and enthalpy of formation of $\mathrm{HOSO}_{4}$ : A quantum chemical study, Chem. Phys. Lett. (submitted).

40 S. Höfener, F. A. Bischoff, A. Glöß, and W. Klopper, Phys. Chem. Chem. Phys. 10, 3390 (2008).

41 K. D. Vogiatzis, A. Mavrandonakis, W. Klopper, and G. E. Froudakis, Ab initio study of the interactions between $\mathrm{CO}_{2}$ and $\mathrm{N}$-containing organic heterocycles, ChemPhysChem (in press).

42 A. Bihlmeier, D. P. Tew, and W. Klopper, J. Chem. Phys. 129, 114303 (2008).

43 Y. Wang, B. J. Braams, J. M. Bowman, S. Carter, and D. P. Tew, J. Chem. Phys. 128, 224314 (2008).

44 F. Claeyssens, J. N. Harveyn, F. R. Manby, R. A. Mata, A. J. Mulholland, K. E. Ranaghan, M. Schütz, S. Thiel, W. Thiel, and H.-J. Werner, Angew. Chem. Int. Ed. 45, 6856 (2006).

45 R. A. Mata, H.-J. Werner, S. Thiel, and W. Thiel, J. Chem. Phys. 128, 025104 (2008).

46 D. P. Tew and W. Klopper, J. Chem. Phys. 123, 074101 (2005). 
47 K. A. Peterson, T. B. Adler, and H.-J. Werner, J. Chem. Phys. 128, 084102 (2008).

48 T. H. Dunning, Jr., J. Chem. Phys. 90, 1007 (1989).

49 R. A. Kendall, T. H. Dunning, Jr., and R. J. Harrison, J. Chem. Phys. 96, 6796 (1992).

50 A. K. Wilson, T. van Mourik, and T. H. Dunning, Jr., J. Mol. Struct.: THEOCHEM 388, 339 (1996).

51 F. Weigend and R. Ahlrichs, Phys. Chem. Chem. Phys. 7, 3297 (2005).

52 D. Bakowies, J. Chem. Phys. 127, 084105 (2007).

53 W. Klopper, B. Ruscic, D. P. Tew, F. A. Bischoff, and S. Wolfsegger, Atomization energies from coupled-cluster calculations augmented with explicitly-correlated perturbation theory, Chem. Phys. (in press).

54 S. Ten-no, Chemical Physics Letters 398, 56 (2004).

55 W. Klopper, Chem. Phys. Lett. 186, 583 (1991).

56 E. F. Valeev, Chem. Phys. Lett. 395, 190 (2004).

57 C. Hättig, Phys. Chem. Chem. Phys. 7, 59 (2005).

58 C. Hättig and F. Weigend, J. Chem. Phys. 113, 5154 (2000).

59 TURBOMOLE v5.10, Turbomole GmbH, Karlsruhe (2008), see http://www.turbomole.com.

60 F. R. Manby, J. Chem. Phys. 119, 4607 (2003).

61 F. Weigend and M. Häser, Theor. Chem. Acc. 97, 331 (1997).

62 F. Weigend, M. Häser, H. Patzelt, and R. Ahlrichs, Chem. Phys. Lett. 294, 143 (1998).

63 F. A. Bischoff, S. Höfener, A. Glöß, and W. Klopper, Theor. Chem. Acc. 121, 11 (2008).

64 F. Weigend, J. Comput. Chem. 29, 167 (2008).

65 F. Weigend, unpublished.

66 S. Ten-no, J. Chem. Phys. 121, 117 (2004).

67 W. Kutzelnigg and W. Klopper, J. Chem. Phys. 94, 1985 (1991).

68 J. Noga, S. Kedžuch, and J. Šimunek, J. Chem. Phys. 127, 034106 (2007).

69 T. Helgaker, W. Klopper, H. Koch, and J. Noga, J. Chem. Phys. 106, 9639 (1997). 
TABLE I: Molecules in the test set and their reference near basis-set limit Hartree-Fock (HF) and MP2 frozen-core correlation energies (in $E_{\mathrm{h}}$ ). B-A denotes the energy difference (in $E_{\mathrm{h}}$ ) between the MP2-F12/2B and MP2-F12/2A frozen-core correlation energies.

\begin{tabular}{|c|c|c|c|c|c|}
\hline $\mathrm{Nr}$ & Molecul & & HF energy & MP2 contribution & $\mathrm{B}-\mathrm{A}$ \\
\hline 1 & $\mathrm{CFN}$ & cyanogen fluoride & -191.7869115 & -0.6734136 & 0.0003255 \\
\hline 2 & $\mathrm{CFN}$ & isocyanogen fluoride & -191.6701799 & -0.6681564 & 0.0003199 \\
\hline 3 & $\mathrm{CF}_{2}$ & singlet difluoromethylene & -236.7797603 & -0.7396863 & 0.0003906 \\
\hline 4 & $\mathrm{CF}_{2} \mathrm{O}$ & carbonyl fluoride & -311.7690426 & -1.0179198 & 0.0005292 \\
\hline 5 & $\mathrm{CF}_{4}$ & tetrafluoromethane & -435.8611009 & -1.3480501 & 0.0007405 \\
\hline 6 & $\mathrm{CHF}$ & singlet fluoromethylene & -137.8254451 & -0.4486484 & 0.0002237 \\
\hline 7 & $\mathrm{CHFO}$ & formyl fluoride & -212.8538870 & -0.7357389 & 0.0003595 \\
\hline 8 & $\mathrm{CHF}_{3}$ & trifluoromethane & -336.9423500 & -1.0664251 & 0.0005611 \\
\hline 9 & $\mathrm{CHN}$ & hydrogen cyanide & -92.9152377 & -0.3871352 & 0.0001662 \\
\hline 10 & $\mathrm{CHN}$ & hydrogen isocyanide & -92.8999603 & -0.3740514 & 0.0001622 \\
\hline 11 & CHNO & cyanic acid & -167.8048239 & -0.6544582 & 0.0002937 \\
\hline 12 & CHNO & isocyanic acid & -167.8447501 & -0.6553452 & 0.0002937 \\
\hline 13 & CHNO & formonitrile oxide & -167.7071831 & -0.6848635 & 0.0002934 \\
\hline 14 & CHNO & isofulminic acid & -167.7092725 & -0.6482486 & 0.0002892 \\
\hline 15 & $\mathrm{CH}_{2}$ & singlet methylene & -38.8959361 & -0.1559390 & 0.0000626 \\
\hline 16 & $\mathrm{CH}_{2} \mathrm{~F}_{2}$ & difluoromethane & -238.0207580 & -0.7832349 & 0.0003889 \\
\hline 17 & $\mathrm{CH}_{2} \mathrm{~N}_{2}$ & cyanamide & -147.9793717 & -0.6195516 & 0.0002591 \\
\hline 18 & $\mathrm{CH}_{2} \mathrm{~N}_{2}$ & 3H-diazirine & -147.8975793 & -0.6301166 & 0.0002591 \\
\hline 19 & $\mathrm{CH}_{2} \mathrm{~N}_{2}$ & diazomethane & -147.9120691 & -0.6318115 & 0.0002512 \\
\hline 20 & $\mathrm{CH}_{2} \mathrm{O}$ & formaldehyde & -113.9230386 & -0.4485051 & 0.0001954 \\
\hline 21 & $\mathrm{CH}_{2} \mathrm{O}$ & hydroxymethylene & -113.8468195 & -0.4358324 & 0.0001911 \\
\hline 22 & $\mathrm{CH}_{2} \mathrm{O}_{2}$ & dioxirane & -188.6951256 & -0.7335711 & 0.0003291 \\
\hline 23 & $\mathrm{CH}_{2} \mathrm{O}_{2}$ & formic acid & -188.8600034 & -0.7188020 & 0.0003283 \\
\hline 24 & $\mathrm{CH}_{2} \mathrm{O}_{3}$ & performic acid & -263.6387395 & -0.9930592 & 0.0004664 \\
\hline 25 & $\mathrm{CH}_{3} \mathrm{~F}$ & fluoromethane & -139.1097750 & -0.5001195 & 0.0002245 \\
\hline 26 & $\mathrm{CH}_{3} \mathrm{~N}$ & methanimine & -94.0762519 & -0.4159873 & 0.0001582 \\
\hline
\end{tabular}




\begin{tabular}{|c|c|c|c|c|c|}
\hline Nr. & Molecule & & HF energy & MP2 contribution & $\mathrm{B}-\mathrm{A}$ \\
\hline 27 & $\mathrm{CH}_{3} \mathrm{NO}$ & formamide & -169.0186910 & -0.6846840 & 0.0002934 \\
\hline 28 & $\mathrm{CH}_{3} \mathrm{NO}_{2}$ & methyl nitrite & -243.7812749 & -0.9679194 & 0.0004309 \\
\hline 29 & $\mathrm{CH}_{3} \mathrm{NO}_{2}$ & nitromethane & -243.7809017 & -0.9795674 & 0.0004314 \\
\hline 30 & $\mathrm{CH}_{4}$ & methane & -40.2170458 & -0.2190333 & 0.0000639 \\
\hline 31 & $\mathrm{CH}_{4} \mathrm{~N}_{2} \mathrm{O}$ & urea & -224.1021999 & -0.9176184 & 0.0004168 \\
\hline 32 & $\mathrm{CH}_{4} \mathrm{O}$ & methanol & -115.1017391 & -0.4851798 & 0.0001915 \\
\hline 33 & $\mathrm{CH}_{5} \mathrm{~N}$ & methylamine & -95.2629113 & -0.4525078 & 0.0001566 \\
\hline 34 & $\mathrm{CO}$ & carbon monoxide & -112.7902518 & -0.4044170 & 0.0001939 \\
\hline 35 & $\mathrm{CO}_{2}$ & carbon dioxide & -187.7244395 & -0.6867786 & 0.0003296 \\
\hline 36 & $\mathrm{C}_{2} \mathrm{~F}_{2}$ & difluoroacetylene (fixed) & -274.5945125 & -0.9134635 & 0.0004456 \\
\hline 37 & $\mathrm{C}_{2} \mathrm{~F}_{4}$ & tetrafluoroethylene & -473.6525592 & -1.5101832 & 0.0008251 \\
\hline 38 & $\mathrm{C}_{2} \mathrm{HF}$ & fluoroacetylene & -175.7291423 & -0.6298874 & 0.0003019 \\
\hline 39 & $\mathrm{C}_{2} \mathrm{HF}_{3}$ & trifluoroethylene & -374.7602074 & -1.2255182 & 0.0006401 \\
\hline 40 & $\mathrm{C}_{2} \mathrm{H}_{2}$ & acetylene & -76.8552215 & -0.3454401 & 0.0001424 \\
\hline 41 & $\mathrm{C}_{2} \mathrm{H}_{2} \mathrm{~F}_{2}$ & 1,1-difluoroethylene & -275.8774293 & -0.9420319 & 0.0004633 \\
\hline 42 & $\mathrm{C}_{2} \mathrm{H}_{2} \mathrm{O}$ & ketene & -151.7979880 & -0.6088949 & 0.0002631 \\
\hline 43 & $\mathrm{C}_{2} \mathrm{H}_{2} \mathrm{O}$ & oxirene & -151.6575846 & -0.6249065 & 0.0002747 \\
\hline 44 & $\mathrm{C}_{2} \mathrm{H}_{2} \mathrm{O}_{2}$ & glyoxal & -226.6972470 & -0.8715650 & 0.0004052 \\
\hline 45 & $\mathrm{C}_{2} \mathrm{H}_{3} \mathrm{~F}$ & fluoroethylene & -176.9713289 & -0.6574949 & 0.0002945 \\
\hline 46 & $\mathrm{C}_{2} \mathrm{H}_{3} \mathrm{FO}$ & acetyl fluoride & -251.9204472 & -0.9258834 & 0.0004359 \\
\hline 47 & $\mathrm{C}_{2} \mathrm{H}_{3} \mathrm{~N}$ & acetonitrile & -131.9837319 & -0.5761492 & 0.0002317 \\
\hline 48 & $\mathrm{C}_{2} \mathrm{H}_{3} \mathrm{~N}$ & methyl isocyanide & -131.9526934 & -0.5641634 & 0.0002287 \\
\hline 49 & $\mathrm{C}_{2} \mathrm{H}_{4}$ & ethylene & -78.0705663 & -0.3726636 & 0.0001305 \\
\hline 50 & $\mathrm{C}_{2} \mathrm{H}_{4} \mathrm{O}$ & acetaldehyde & -152.9890345 & -0.6397856 & 0.0002658 \\
\hline 51 & $\mathrm{C}_{2} \mathrm{H}_{4} \mathrm{O}$ & oxirane & -152.9404392 & -0.6484923 & 0.0002676 \\
\hline 52 & $\mathrm{C}_{2} \mathrm{H}_{4} \mathrm{O}_{2}$ & acetic acid & -227.9243915 & -0.9094852 & 0.0004037 \\
\hline 53 & $\mathrm{C}_{2} \mathrm{H}_{4} \mathrm{O}_{2}$ & methyl formate & -227.8981132 & -0.9076775 & 0.0004029 \\
\hline 54 & $\mathrm{C}_{2} \mathrm{H}_{5} \mathrm{~F}$ & fluoroethane & -178.1675871 & -0.6917162 & 0.0002950 \\
\hline 55 & $\mathrm{C}_{2} \mathrm{H}_{5} \mathrm{~N}$ & aziridine & -133.1016627 & -0.6164873 & 0.0002303 \\
\hline
\end{tabular}




\begin{tabular}{|c|c|c|c|c|c|}
\hline Nr. & Molecule & & HF energy & MP2 contribution & $\mathrm{B}-\mathrm{A}$ \\
\hline 56 & $\mathrm{C}_{2} \mathrm{H}_{6}$ & ethane & -79.2666426 & -0.4096202 & 0.0001304 \\
\hline 57 & $\mathrm{C}_{2} \mathrm{H}_{6} \mathrm{O}$ & dimethyl ether & -154.1409915 & -0.6737526 & 0.0002640 \\
\hline 58 & $\mathrm{C}_{2} \mathrm{H}_{6} \mathrm{O}$ & ethanol & -154.1578815 & -0.6775961 & 0.0002612 \\
\hline 59 & $\mathrm{C}_{2} \mathrm{~N}_{2}$ & cyanogen & -184.6613685 & -0.7549829 & 0.0003278 \\
\hline 60 & $\mathrm{C}_{3} \mathrm{H}_{3} \mathrm{~N}$ & acrylonitrile & -169.8386596 & -0.7337807 & 0.0002993 \\
\hline 61 & $\mathrm{C}_{3} \mathrm{H}_{4}$ & allene & -115.9147707 & -0.5310153 & 0.0001983 \\
\hline 62 & $\mathrm{C}_{3} \mathrm{H}_{4}$ & cyclopropene & -115.8742896 & -0.5414329 & 0.0002050 \\
\hline 63 & $\mathrm{C}_{3} \mathrm{H}_{4}$ & propyne & -115.9172745 & -0.5363030 & 0.0002073 \\
\hline 64 & $\mathrm{C}_{3} \mathrm{H}_{6}$ & cyclopropane & -117.1107221 & -0.5733140 & 0.0002021 \\
\hline 65 & $\mathrm{C}_{3} \mathrm{H}_{6}$ & propene & -117.1261419 & -0.5657594 & 0.0001989 \\
\hline 66 & $\mathrm{C}_{3} \mathrm{H}_{8}$ & propane & -118.3175998 & -0.6026590 & 0.0001994 \\
\hline 67 & $\mathrm{C}_{3} \mathrm{O}_{2}$ & carbon suboxide (fixed) & -263.3970576 & -1.0186298 & 0.0004355 \\
\hline 68 & $\mathrm{C}_{4} \mathrm{H}_{4}$ & butatriene & -153.7576701 & -0.6937222 & 0.0002723 \\
\hline 69 & $\mathrm{C}_{4} \mathrm{H}_{4}$ & cyclobutadiene & -153.7077028 & -0.7045815 & 0.0002847 \\
\hline 70 & $\mathrm{C}_{4} \mathrm{H}_{4}$ & tetrahedrane & -153.6640182 & -0.7165988 & 0.0002872 \\
\hline 71 & $\mathrm{C}_{4} \mathrm{H}_{4}$ & vinylacetylene & -153.7756023 & -0.6944420 & 0.0002742 \\
\hline 72 & $\mathrm{C}_{4} \mathrm{~N}_{2}$ & dicyanoacetylene (fixed) & -260.3699559 & -1.0809805 & 0.0004247 \\
\hline 73 & $\mathrm{FH}$ & hydrogen fluoride & -100.0707863 & -0.3197419 & 0.0001559 \\
\hline 74 & FHO & hypofluorous acid & -174.8227081 & -0.5880740 & 0.0002847 \\
\hline 75 & $\mathrm{FHO}_{2}$ & fluoroperoxide & -249.6239480 & -0.8653412 & 0.0004190 \\
\hline 76 & $\mathrm{FH}_{2} \mathrm{~N}$ & monofluoroamine & -155.0409719 & -0.5476942 & 0.0002515 \\
\hline 77 & $\mathrm{FH}_{3} \mathrm{~N}_{2}$ & fluorohydrazine & -210.0684626 & -0.7849995 & 0.0003509 \\
\hline 78 & $\mathrm{FNO}$ & nitrosyl fluoride & -228.7304686 & -0.8025419 & 0.0003847 \\
\hline 79 & $\mathrm{~F}_{2}$ & difluorine & -198.7731963 & -0.6118360 & 0.0003137 \\
\hline 80 & $\mathrm{~F}_{2} \mathrm{~N}_{2}$ & difluorodiazene (cis) & -307.7266920 & -1.0469854 & 0.0005221 \\
\hline 81 & $\mathrm{~F}_{2} \mathrm{~N}_{2}$ & difluorodiazene (trans) & -307.7288330 & -1.0425389 & 0.0005257 \\
\hline 82 & $\mathrm{~F}_{2} \mathrm{O}$ & difluorine monoxide & -273.5865780 & -0.8838321 & 0.0004496 \\
\hline 83 & $\mathrm{~F}_{2} \mathrm{O}_{2}$ & perfluoroperoxide & -348.3742509 & -1.1833499 & 0.0005845 \\
\hline 84 & $\mathrm{~F}_{3} \mathrm{~N}$ & trifluoroamine & -352.7142390 & -1.1280514 & 0.0005922 \\
\hline
\end{tabular}




\begin{tabular}{|c|c|c|c|c|c|}
\hline \multicolumn{3}{|c|}{ Nr. Molecule } & \multirow{2}{*}{$\frac{\text { HF energy }}{-129.8492567}$} & \multirow{2}{*}{ MP2 contribution } & \multirow{2}{*}{$\frac{\mathrm{B}-\mathrm{A}}{0.0002205}$} \\
\hline 85 & HNO & nitrosylhydride & & & \\
\hline 86 & $\mathrm{HNO}_{2}$ & nitrous acid (cis) & -204.7423915 & -0.7760086 & 0.0003540 \\
\hline 87 & $\mathrm{HNO}_{2}$ & nitrous acid (trans) & -204.7415982 & -0.7773909 & 0.0003529 \\
\hline 88 & $\mathrm{HNO}_{2}$ & nitrous acid, $\mathrm{H}-\mathrm{NO}_{2}$ & -204.7219722 & -0.7922894 & 0.0003530 \\
\hline 89 & $\mathrm{HNO}_{3}$ & nitric acid & -279.5864471 & -1.0623180 & 0.0004918 \\
\hline 90 & $\mathrm{HN}_{3}$ & hydrogen azide & -163.9172349 & -0.6876363 & 0.0002793 \\
\hline 91 & $\mathrm{H}_{2} \mathrm{~N}_{2}$ & diazene (cis) & -110.0396202 & -0.4608058 & 0.0001852 \\
\hline 92 & $\mathrm{H}_{2} \mathrm{~N}_{2}$ & diazene (trans) & -110.0491822 & -0.4603129 & 0.0001849 \\
\hline 93 & $\mathrm{H}_{2} \mathrm{~N}_{2}$ & diazene (iso) & -110.0206290 & -0.4448095 & 0.0001875 \\
\hline 94 & $\mathrm{H}_{2} \mathrm{~N}_{2} \mathrm{O}$ & nitrosamide & -184.9201527 & -0.7368870 & 0.0003205 \\
\hline 95 & $\mathrm{H}_{2} \mathrm{O}$ & water & -76.0673059 & -0.3006048 & 0.0001233 \\
\hline 96 & $\mathrm{H}_{2} \mathrm{O}_{2}$ & hydrogen peroxide & -150.8517704 & -0.5701178 & 0.0002531 \\
\hline 97 & $\mathrm{H}_{3} \mathrm{~N}$ & ammonia & -56.2247904 & -0.2646166 & 0.0000894 \\
\hline 98 & $\mathrm{H}_{3} \mathrm{NO}$ & ammonia oxide & -131.0171087 & -0.5309810 & 0.0002275 \\
\hline 99 & $\mathrm{H}_{3} \mathrm{NO}$ & hydroxylamine & -131.0554068 & -0.5317602 & 0.0002187 \\
\hline 100 & $\mathrm{H}_{4} \mathrm{~N}_{2}$ & hydrazine & -111.2361412 & -0.4961172 & 0.0001834 \\
\hline 101 & $\mathrm{~N}_{2}$ & dinitrogen & -108.9925134 & -0.4215422 & 0.0001879 \\
\hline 102 & $\mathrm{~N}_{2} \mathrm{O}$ & nitrous oxide & -183.7656610 & -0.7258487 & 0.0003154 \\
\hline 103 & $\mathrm{~N}_{2} \mathrm{O}_{3}$ & dinitrogen trioxide & -333.3899231 & -1.2928074 & 0.0005934 \\
\hline 104 & $\mathrm{~N}_{2} \mathrm{O}_{4}$ & dinitrogen tetraoxide & -408.2303052 & -1.5951352 & 0.0007361 \\
\hline 105 & $\mathrm{O}_{3}$ & ozone & -224.3637281 & -0.8810446 & 0.0003830 \\
\hline 106 & $\mathrm{H}_{2}$ & dihydrogen & -1.1336066 & -0.0342510 & 0.0000066 \\
\hline 107 & & carbon & -37.6886122 & -0.0818513 & 0.0000417 \\
\hline 108 & & nitrogen & -54.4009236 & -0.1137928 & 0.0000000 \\
\hline 109 & & oxygen & -74.8093817 & -0.1792499 & 0.0001047 \\
\hline 110 & & fluorine & -99.4093241 & -0.2477403 & 0.0001465 \\
\hline
\end{tabular}


TABLE II: Statistical measures of the errors per valence electron in the fc-MP2-F12 correlation energies $(\mathrm{kJ} / \mathrm{mol})$ arising from incompleteness in the orbital, CABS and density-fitting basis sets.

\begin{tabular}{lcccrrr}
\hline \hline Error & $\bar{\Delta}$ & $\sigma$ & $\Delta_{\text {abs. }}$ & $\Delta_{\text {rms }}$ & $\Delta_{\text {max. }}$ & Molecule \\
\hline Orb. & 0.0421 & 0.0096 & 0.0421 & 0.0432 & 0.0586 & $79 \mathrm{~F}_{2}$ \\
CABS & -0.0024 & 0.0005 & 0.0024 & 0.0024 & -0.0036 & $101 \mathrm{~N}_{2}$ \\
RI-MP2 & -0.0011 & 0.0004 & 0.0011 & 0.0012 & -0.0028 & $106 \mathrm{H}_{2}$ \\
RI-F12 & -0.0012 & 0.0001 & 0.0012 & 0.0012 & -0.0015 & $15 \mathrm{CH}_{2}$ \\
RI-Tot. & -0.0001 & 0.0001 & 0.0001 & 0.0001 & -0.0003 & $84 \mathrm{~F}_{3} \mathrm{~N}$ \\
RI-JK & 0.0001 & 0.0000 & 0.0001 & 0.0001 & 0.0002 & $4 \mathrm{CF}_{2} \mathrm{O}$ \\
\hline Fixed-Inv & 0.0042 & 0.0019 & 0.0042 & 0.0046 & 0.0086 & $5 \mathrm{CF}_{4}$ \\
\hline \hline
\end{tabular}


TABLE III: Statistical measures for the error per valence electron in the Hartree-Fock energy $(\mathrm{kJ} / \mathrm{mol})$.

\begin{tabular}{lcccccc}
\hline \hline Basis & $\bar{\Delta}$ & $\sigma$ & $\Delta_{\text {abs. }}$ & $\Delta_{\text {rms }}$ & $\Delta_{\text {max. }}$ & \\
\hline cc-pVDZ-F12 & 4.05 & 0.53 & 4.05 & 4.08 & 7.42 & $106 \mathrm{H}_{2}$ \\
cc-pVTZ-F12 & 0.57 & 0.15 & 0.57 & 0.59 & 0.91 & $73 \mathrm{FH}$ \\
cc-pVQZ-F12 & 0.06 & 0.01 & 0.06 & 0.06 & 0.09 & $73 \mathrm{FH}$ \\
aug-cc-pVDZ & 8.74 & 2.13 & 8.74 & 9.00 & 13.98 & $79 \mathrm{~F}_{2}$ \\
aug-cc-pVTZ & 2.14 & 0.58 & 2.14 & 2.22 & 3.49 & $79 \mathrm{~F}_{2}$ \\
aug-cc-pVQZ & 0.45 & 0.14 & 0.45 & 0.47 & 0.82 & $79 \mathrm{~F}_{2}$ \\
aug-cc-pV5Z & 0.05 & 0.01 & 0.05 & 0.05 & 0.07 & $79 \mathrm{~F}_{2}$ \\
def2-TZVPP & 1.35 & 0.25 & 1.35 & 1.37 & 1.78 & $84 \mathrm{~F}_{3} \mathrm{~N}$ \\
def2-QZVPP & 0.17 & 0.04 & 0.17 & 0.17 & 0.27 & $98 \mathrm{H}_{3} \mathrm{NO}$ \\
\hline \hline
\end{tabular}


TABLE IV: Statistical measures for the error per valence electron in the Hartree-Fock energy when the CABS singles correction is included $(\mathrm{kJ} / \mathrm{mol})$.

\begin{tabular}{lcccccc}
\hline \hline Basis & $\bar{\Delta}$ & $\sigma$ & $\Delta_{\text {abs. }}$ & $\Delta_{\text {rms }}$ & $\Delta_{\text {max. }}$ & Molecule \\
\hline cc-pVDZ-F12 & 0.93 & 0.09 & 0.93 & 0.93 & 1.47 & $106 \mathrm{H}_{2}$ \\
cc-pVTZ-F12 & 0.22 & 0.04 & 0.22 & 0.22 & 0.32 & $79 \mathrm{~F}_{2}$ \\
cc-pVQZ-F12 & 0.03 & 0.01 & 0.03 & 0.04 & 0.06 & $79 \mathrm{~F}_{2}$ \\
aug-cc-pVDZ & 1.21 & 0.17 & 1.21 & 1.23 & 1.71 & $79 \mathrm{~F}_{2}$ \\
aug-cc-pVTZ & 0.68 & 0.09 & 0.68 & 0.68 & 0.89 & $79 \mathrm{~F}_{2}$ \\
aug-cc-pVQZ & 0.16 & 0.04 & 0.16 & 0.17 & 0.27 & $79 \mathrm{~F}_{2}$ \\
aug-cc-pV5Z & 0.03 & 0.01 & 0.03 & 0.03 & 0.05 & $79 \mathrm{~F}_{2}$ \\
def2-TZVPP & 0.43 & 0.06 & 0.43 & 0.43 & 0.64 & $98 \mathrm{H}_{3} \mathrm{NO}$ \\
def2-QZVPP & 0.07 & 0.02 & 0.07 & 0.07 & 0.12 & $98 \mathrm{H}_{3} \mathrm{NO}$ \\
\hline \hline
\end{tabular}


TABLE V: Statistical measures for the error per valence electron $(\mathrm{kJ} / \mathrm{mol})$ in the fc-MP2-F12 correlation energy (invariant formulation) as well as in the extrapolated fc-MP2 correlation energy.

\begin{tabular}{lcccccc}
\hline \hline Basis & $\bar{\Delta}$ & $\sigma$ & $\Delta_{\text {abs. }}$ & $\Delta_{\text {rms }}$ & $\Delta_{\text {max. }}$ & Molecule \\
\hline cc-pVDZ-F12 & 0.97 & 0.24 & 0.97 & 1.00 & 1.75 & $73 \mathrm{FH}$ \\
cc-pVTZ-F12 & 0.26 & 0.08 & 0.26 & 0.27 & 0.41 & $79 \mathrm{~F}_{2}$ \\
cc-pVQZ-F12 & 0.07 & 0.02 & 0.07 & 0.07 & 0.10 & $79 \mathrm{~F}_{2}$ \\
aug-cc-pVDZ & 1.71 & 0.22 & 1.71 & 1.73 & 2.19 & $79 \mathrm{~F}_{2}$ \\
aug-cc-pVTZ & 0.63 & 0.18 & 0.63 & 0.65 & 1.02 & $79 \mathrm{~F}_{2}$ \\
aug-cc-pVQZ & 0.23 & 0.07 & 0.23 & 0.24 & 0.40 & $79 \mathrm{~F}_{2}$ \\
aug-cc-pV5Z & 0.09 & 0.03 & 0.09 & 0.09 & 0.17 & $79 \mathrm{~F}_{2}$ \\
def2-TZVPP & 1.15 & 0.34 & 1.15 & 1.19 & 2.00 & $73 \mathrm{FH}$ \\
def2-QZVPP & 0.44 & 0.15 & 0.44 & 0.47 & 0.79 & $79 \mathrm{~F}_{2}$ \\
aug-(TQ) & 0.64 & 0.27 & 0.64 & 0.69 & 1.30 & $79 \mathrm{~F}_{2}$ \\
aug-(Q5) & 0.15 & 0.05 & 0.15 & 0.16 & 0.25 & $5 \mathrm{CF}_{4}$ \\
\hline \hline
\end{tabular}


TABLE VI: Statistical measures for the error per valence electron in the total fc-MP2 electronic energy $(\mathrm{kJ} / \mathrm{mol})$.

\begin{tabular}{lcccccc}
\hline \hline Basis & $\bar{\Delta}$ & $\sigma$ & $\Delta_{\text {abs. }}$ & $\Delta_{\text {rms }}$ & $\Delta_{\text {max. }}$ & Molecule \\
\hline cc-pVDZ-F12 & 1.90 & 0.30 & 1.90 & 1.92 & 3.00 & $73 \mathrm{FH}$ \\
cc-pVTZ-F12 & 0.48 & 0.12 & 0.48 & 0.49 & 0.73 & $79 \mathrm{~F}_{2}$ \\
cc-pVQZ-F12 & 0.10 & 0.03 & 0.10 & 0.10 & 0.16 & $79 \mathrm{~F}_{2}$ \\
aug-cc-pVDZ & 2.93 & 0.36 & 2.93 & 2.95 & 3.90 & $79 \mathrm{~F}_{2}$ \\
aug-cc-pVTZ & 1.30 & 0.26 & 1.30 & 1.33 & 1.91 & $79 \mathrm{~F}_{2}$ \\
aug-cc-pVQZ & 0.39 & 0.11 & 0.39 & 0.41 & 0.66 & $79 \mathrm{~F}_{2}$ \\
aug-cc-pV5Z & 0.12 & 0.04 & 0.12 & 0.13 & 0.22 & $79 \mathrm{~F}_{2}$ \\
def2-TZVPP & 1.57 & 0.38 & 1.57 & 1.62 & 2.58 & $73 \mathrm{FH}$ \\
def2-QZVPP & 0.51 & 0.17 & 0.51 & 0.53 & 0.92 & $79 \mathrm{~F}_{2}$ \\
\hline \hline
\end{tabular}


TABLE VII: Statistical measures for the error per valence electron $(\mathrm{kJ} / \mathrm{mol})$ in the correlation contributions to the atomisation energies (invariant formulation).

\begin{tabular}{lcccccc}
\hline \hline Basis & $\bar{\Delta}$ & $\sigma$ & $\Delta_{\text {abs. }}$ & $\Delta_{\text {rms }}$ & $\Delta_{\text {max. }}$ & Molecule \\
\hline cc-pVDZ-F12 & 0.21 & 0.31 & 0.31 & 0.37 & 0.84 & $105 \mathrm{O}_{3}$ \\
cc-pVTZ-F12 & 0.08 & 0.04 & 0.08 & 0.09 & 0.17 & $105 \mathrm{O}_{3}$ \\
cc-pVQZ-F12 & 0.02 & 0.01 & 0.02 & 0.02 & 0.04 & $105 \mathrm{O}_{3}$ \\
aug-cc-pVDZ & 0.31 & 0.35 & 0.37 & 0.47 & 1.04 & $105 \mathrm{O}_{3}$ \\
aug-cc-pVTZ & 0.15 & 0.07 & 0.15 & 0.16 & 0.28 & $83 \mathrm{~F}_{2} \mathrm{O}_{2}$ \\
aug-cc-pVQZ & 0.12 & 0.02 & 0.12 & 0.12 & 0.17 & $67 \mathrm{C}_{3} \mathrm{O}_{2}$ \\
aug-cc-pV5Z & 0.06 & 0.01 & 0.06 & 0.06 & 0.08 & $67 \mathrm{C}_{3} \mathrm{O}_{2}$ \\
def2-TZVPP & 0.12 & 0.17 & 0.17 & 0.21 & 0.49 & $5 \mathrm{CF}_{4}$ \\
def2-QZVPP & 0.11 & 0.05 & 0.11 & 0.12 & 0.23 & $5 \mathrm{CF}_{4}$ \\
aug-(TQ) & -0.09 & 0.08 & 0.10 & 0.12 & -0.38 & $101 \mathrm{~N}_{2}$ \\
aug-(Q5) & -0.09 & 0.03 & 0.09 & 0.10 & -0.17 & $90 \mathrm{HN}_{3}$ \\
\hline \hline
\end{tabular}


TABLE VIII: Statistical measures for the error per valence electron $(\mathrm{kJ} / \mathrm{mol})$ in the atomisation energies (Hartree-Fock plus frozen-core correlation contribution plus CABS singles).

\begin{tabular}{lrrrrrr}
\hline \hline Basis & $\bar{\Delta}$ & $\sigma$ & $\Delta_{\text {abs. }}$ & $\Delta_{\text {rms }}$ & $\Delta_{\text {max. }}$ & Molecule \\
\hline cc-pVDZ-F12 & -0.33 & 0.34 & 0.35 & 0.47 & -1.84 & $106 \mathrm{H}_{2}$ \\
cc-pVTZ-F12 & -0.02 & 0.04 & 0.03 & 0.04 & -0.18 & $106 \mathrm{H}_{2}$ \\
cc-pVQZ-F12 & 0.01 & 0.01 & 0.01 & 0.01 & -0.03 & $106 \mathrm{H}_{2}$ \\
aug-cc-pVDZ & -0.21 & 0.33 & 0.29 & 0.39 & -2.10 & $106 \mathrm{H}_{2}$ \\
aug-cc-pVTZ & 0.02 & 0.10 & 0.08 & 0.11 & -0.64 & $106 \mathrm{H}_{2}$ \\
aug-cc-pVQZ & 0.09 & 0.04 & 0.10 & 0.10 & -0.16 & $106 \mathrm{H}_{2}$ \\
aug-cc-pV5Z & 0.05 & 0.01 & 0.05 & 0.06 & 0.08 & $67 \mathrm{C}_{3} \mathrm{O}_{2}$ \\
def2-TZVPP & -0.04 & 0.23 & 0.17 & 0.23 & -0.93 & $106 \mathrm{H}_{2}$ \\
def2-QZVPP & 0.08 & 0.06 & 0.09 & 0.10 & 0.21 & $5 \mathrm{CF}_{4}$ \\
\hline \hline
\end{tabular}




\section{Figure Captions}

FIG. 1 Mean errors per valence electron $(\mathrm{kJ} / \mathrm{mol})$ in the HF energy as a function of the size of the basis. $(\times=\mathrm{DZ}, \bullet=\mathrm{TZ}, \square=\mathrm{QZ}, \diamond=5 \mathrm{Z}$.)

FIG. 2 Performance of basis sets and extrapolation methods for absolute correlation energies.

FIG. 3 Mean errors per valence electron $(\mathrm{kJ} / \mathrm{mol})$ in the absolute correlation energy as a function of the size of the basis. $(\times=\mathrm{DZ}, \bullet=\mathrm{TZ}, \square=\mathrm{QZ}, \diamond=5 \mathrm{Z}$.)

FIG. 4 Performance of basis sets and extrapolation methods for atomisation energies.

FIG. 5 Mean errors per valence electron $(\mathrm{kJ} / \mathrm{mol})$ in the correlation contribution to the atomisation energy as a function of the size of the basis. $(\times=\mathrm{DZ}, \bullet=\mathrm{TZ}, \square=\mathrm{QZ}, \diamond=$ 5Z.)

FIG. 6 Mean errors per valence electron $(\mathrm{kJ} / \mathrm{mol})$ in the total atomisation energy (HartreeFock plus correlation contribution plus CABS singles) as a function of the size of the basis. $(\times=\mathrm{DZ}, \bullet=\mathrm{TZ}, \square=\mathrm{QZ}, \diamond=5 \mathrm{Z}$.) 


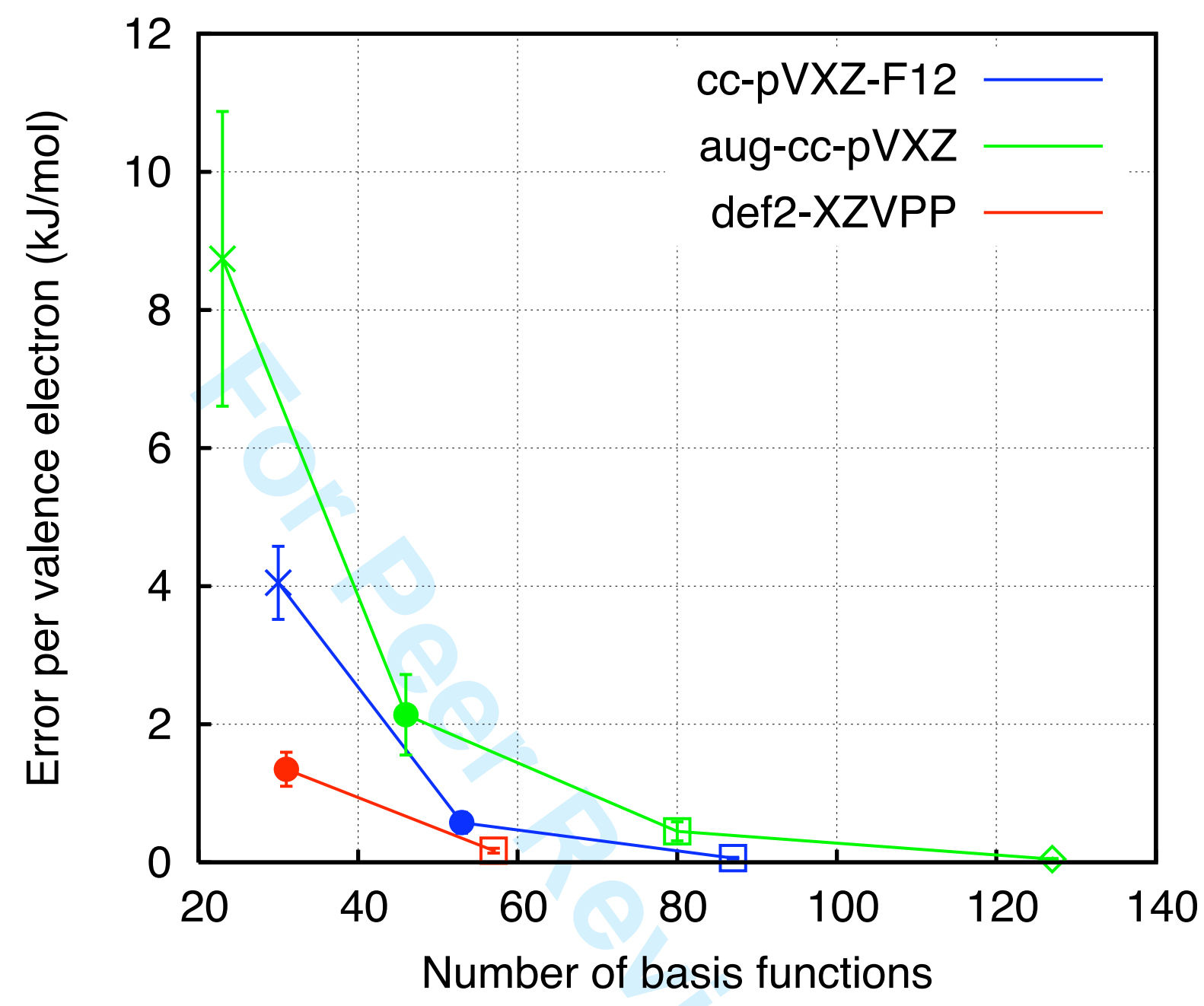

FIG. 1: Bischoff al. 


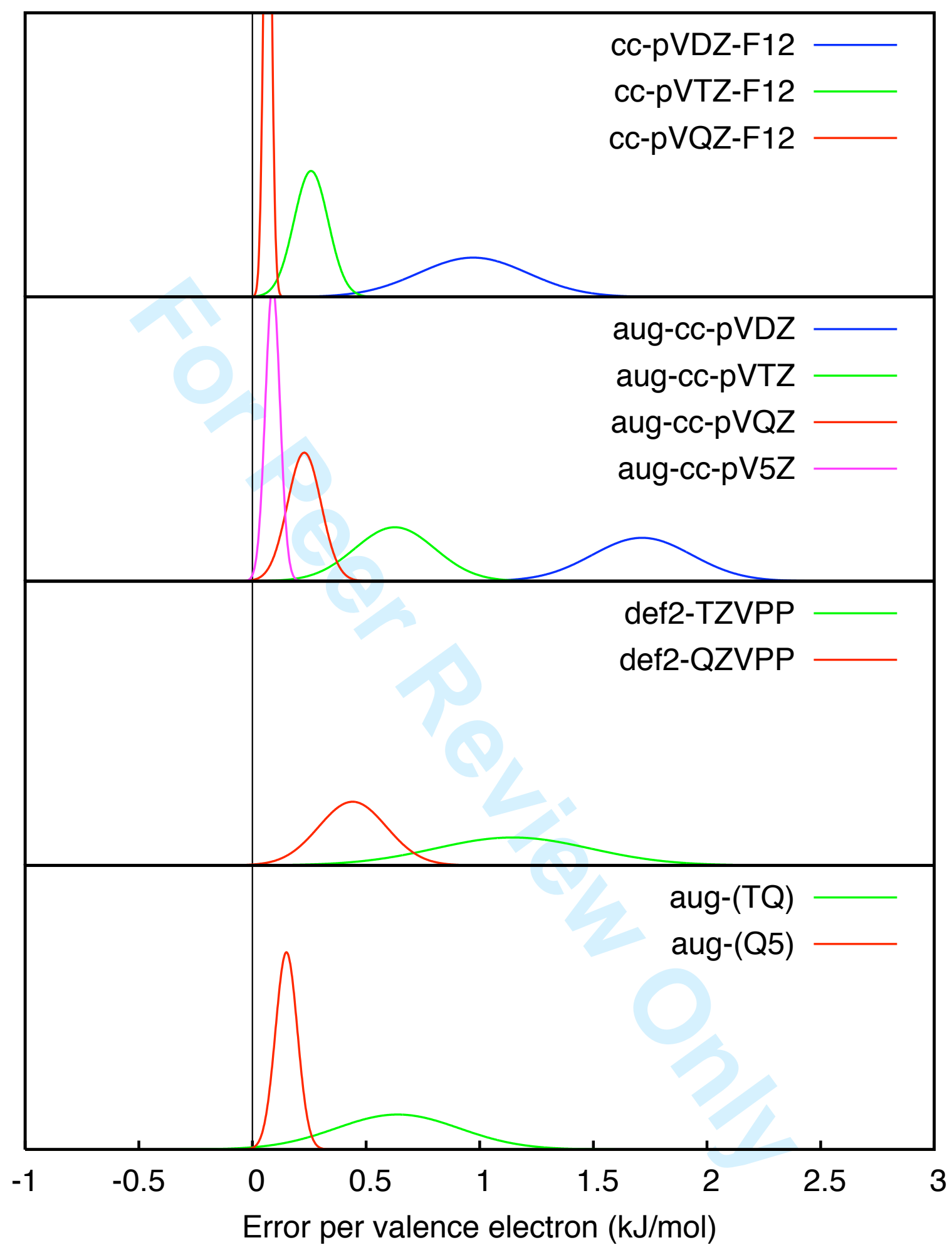




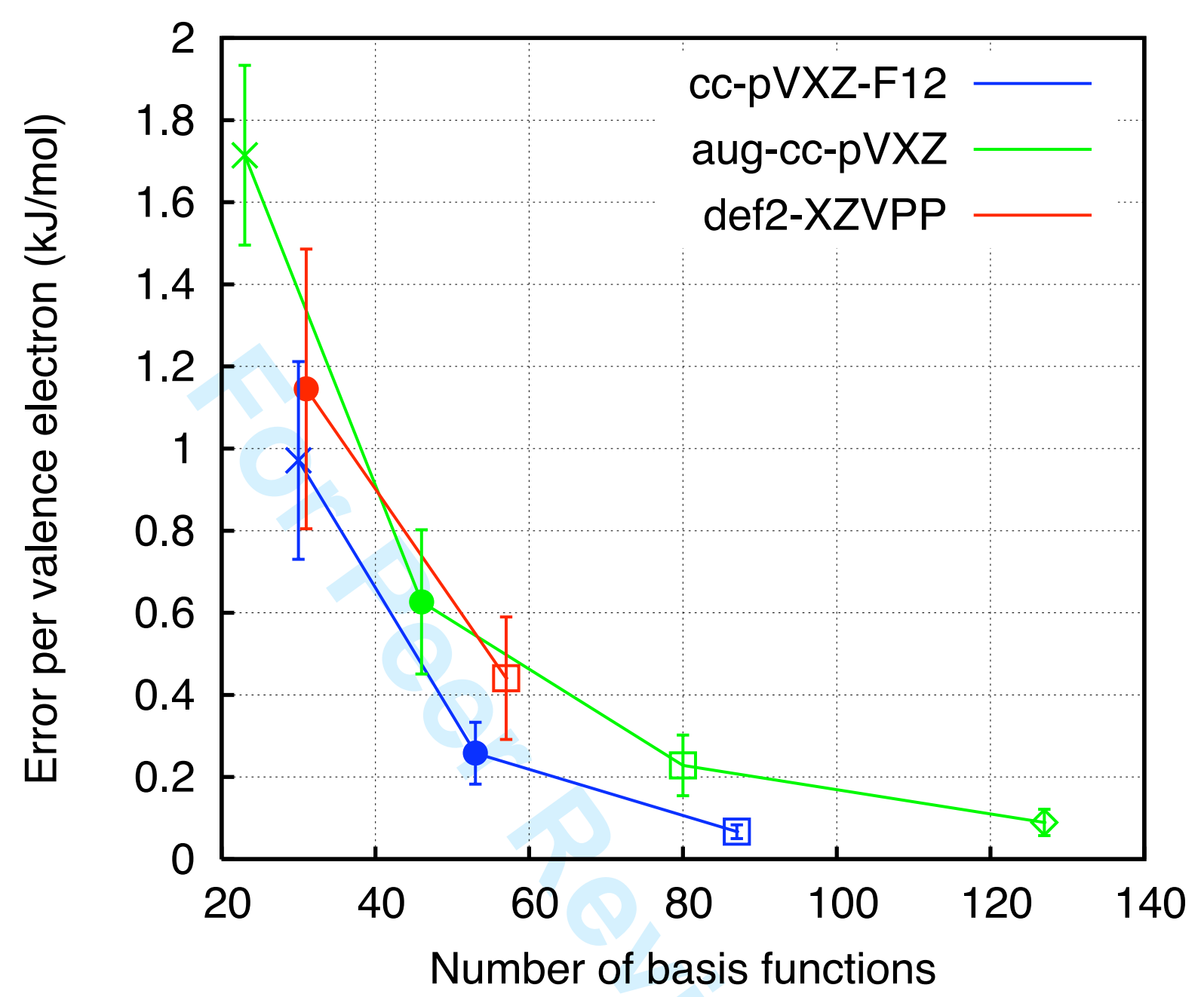

FIG. 3: Bischoff al. 


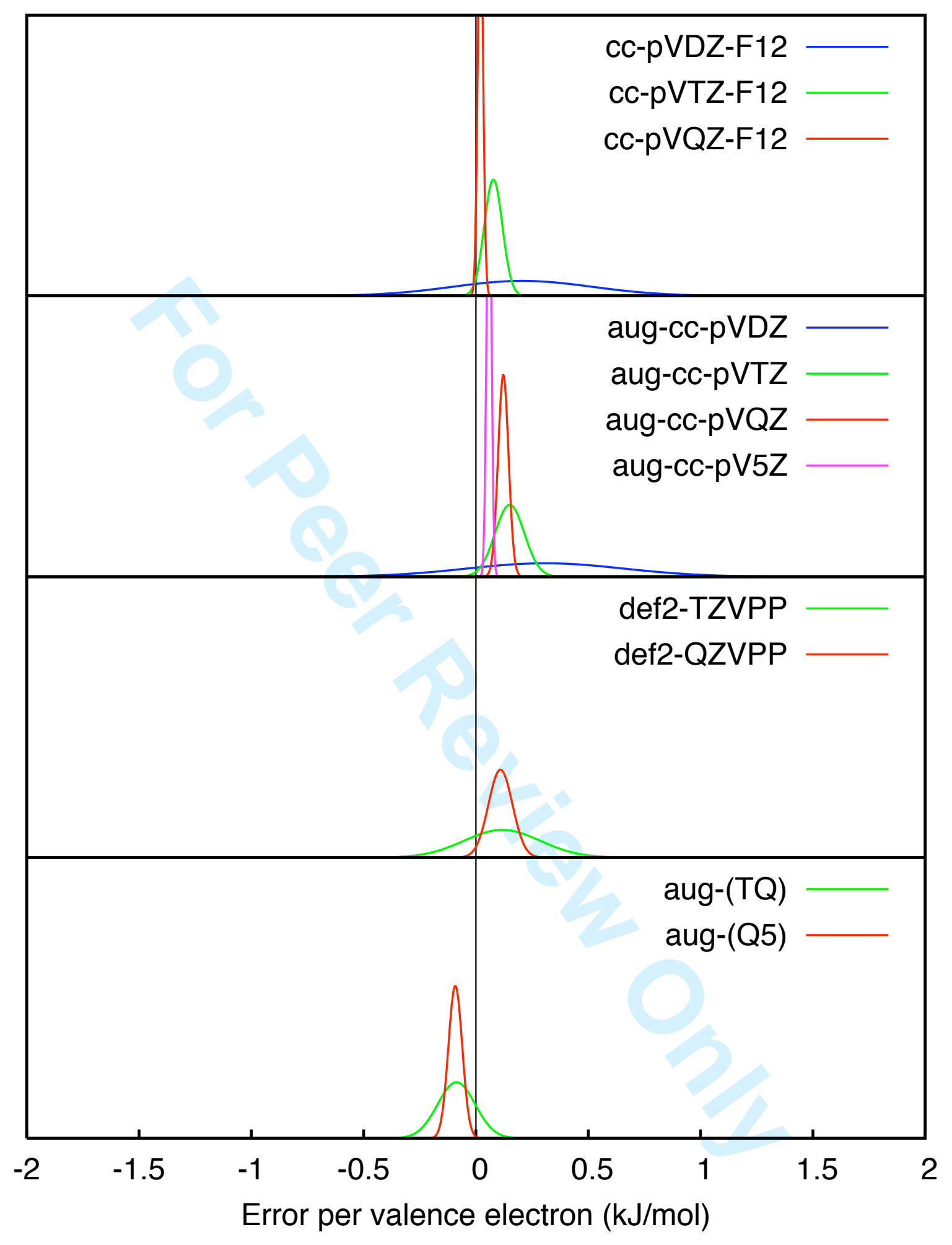




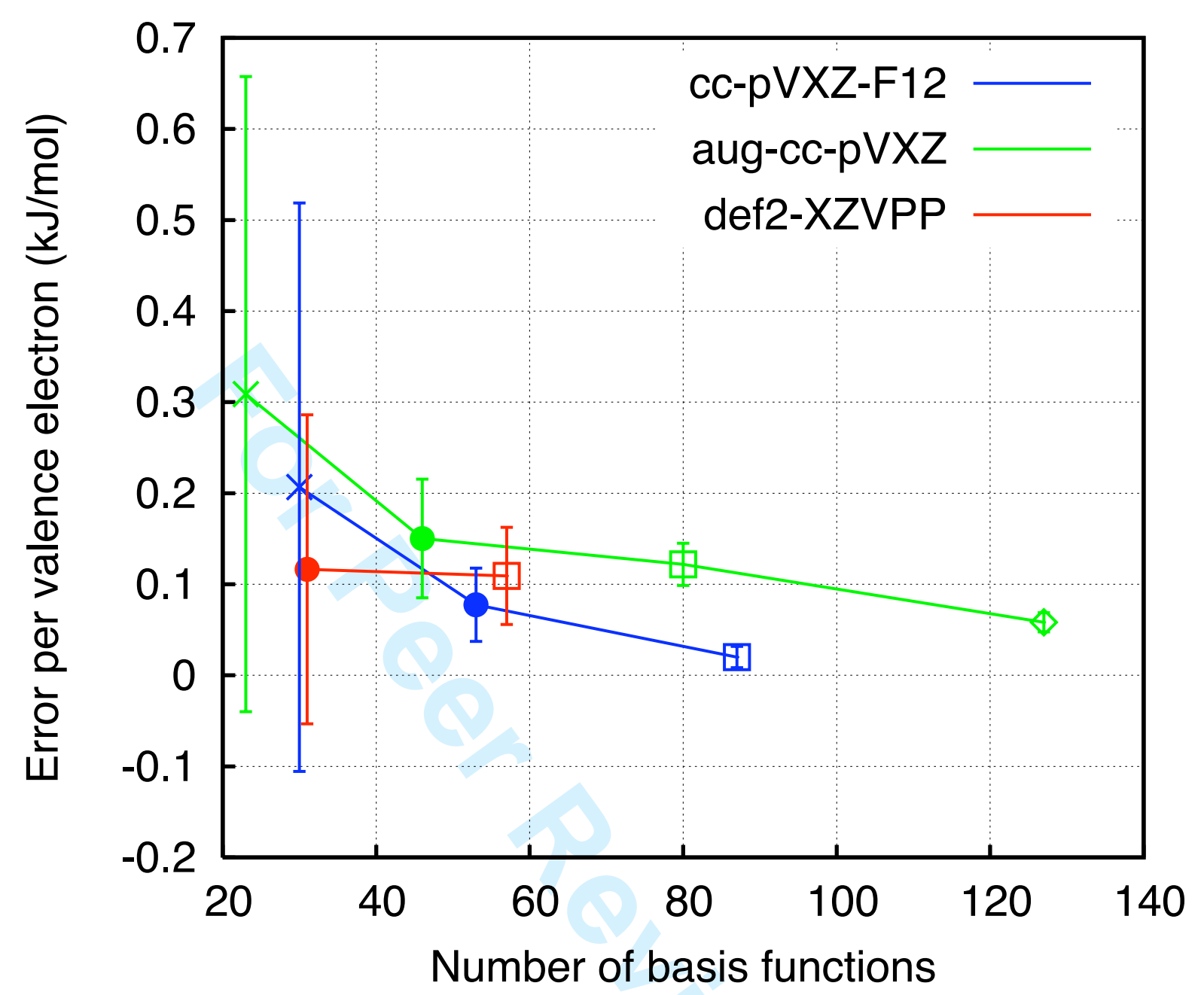

FIG. 5: Bischoff al. 


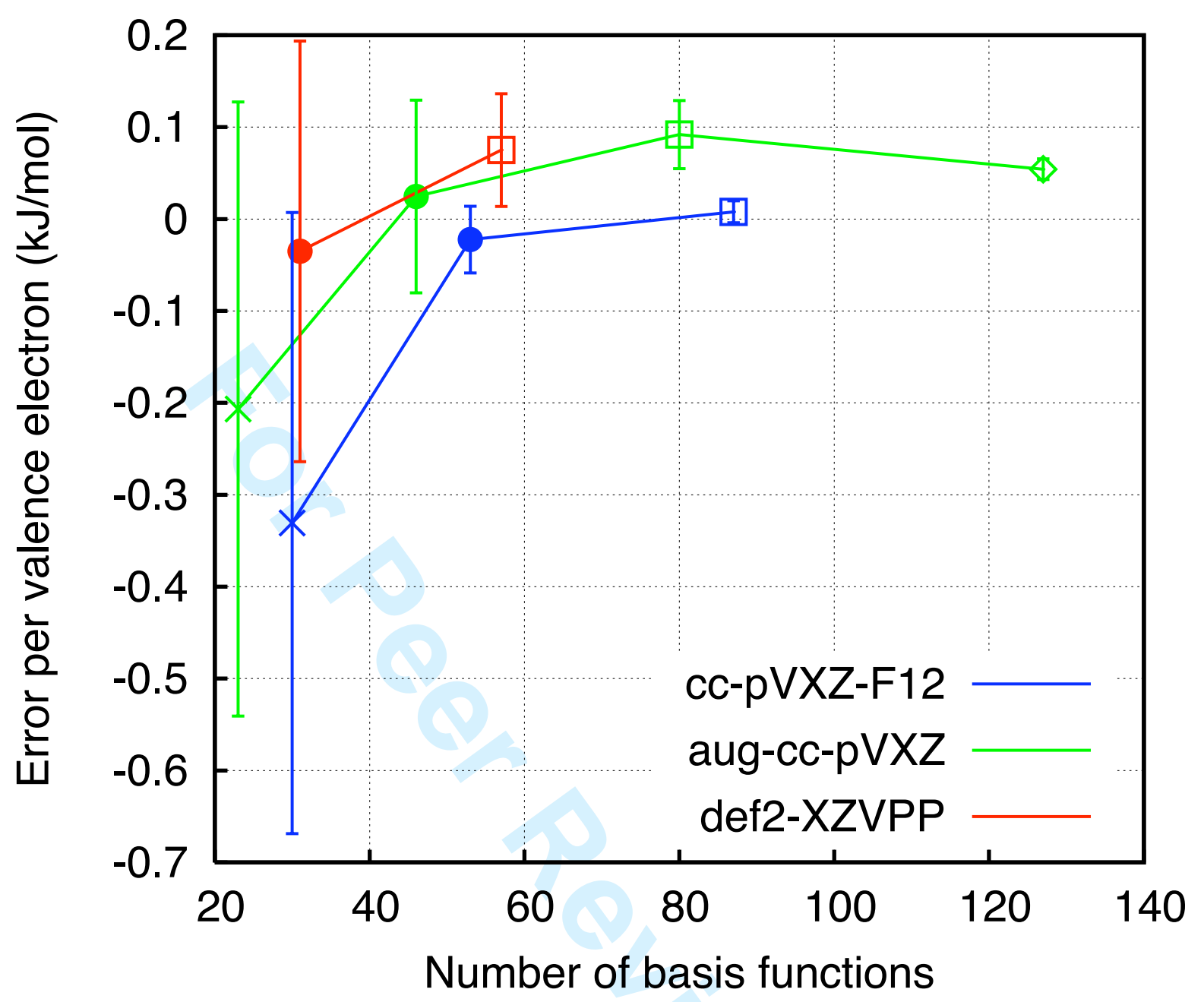

FIG. 6: Bischoff al. 


\section{Assessment of basis sets for F12 explicitly-correlated molecular electronic-structure methods}

\section{Supplementary Information}

Florian A. Bischoff, Sandra Wolfsegger, David P. Tew and Wim Klopper* Lehrstuhl für Theoretische Chemie, Institut für Physikalische Chemie, Universität Karlsruhe (TH), D-76128 Karlsruhe, Germany

(Dated: December 16, 2008)

\footnotetext{
*E-mail: klopper@chem-bio.uni-karlsruhe.de. Fax: +49-721-6083319.
} 
TABLE I: Statistical measures for the error per valence electron $(\mathrm{kJ} / \mathrm{mol})$ in the fc-MP2-F12 correlation energy (fixed-amplitude method).

\begin{tabular}{llllccc}
\hline \hline Basis & $\bar{\Delta}$ & $\sigma$ & $\Delta_{\text {abs. }}$ & $\Delta_{\text {rms }}$ & $\Delta_{\text {max. }}$ Molecule \\
\hline cc-pVDZ-F12 & 1.43 & 0.50 & 1.43 & 1.52 & 2.67 & $79 \mathrm{~F}_{2}$ \\
cc-pVTZ-F12 & 0.35 & 0.13 & 0.35 & 0.38 & 0.64 & $79 \mathrm{~F}_{2}$ \\
cc-pVQZ-F12 & 0.08 & 0.02 & 0.08 & 0.09 & 0.14 & $79 \mathrm{~F}_{2}$ \\
aug-cc-pVDZ & 2.63 & 0.23 & 2.63 & 2.64 & 3.21 & $72 \mathrm{C}_{4} \mathrm{~N}_{2}$ \\
aug-cc-pVTZ & 0.79 & 0.22 & 0.79 & 0.82 & 1.31 & $79 \mathrm{~F}_{2}$ \\
aug-cc-pVQZ & 0.30 & 0.10 & 0.30 & 0.31 & 0.54 & $79 \mathrm{~F}_{2}$ \\
aug-cc-pV5Z & 0.12 & 0.05 & 0.12 & 0.13 & 0.23 & $79 \mathrm{~F}_{2}$ \\
def2-TZVPP & 1.38 & 0.40 & 1.38 & 1.43 & 2.38 & $79 \mathrm{~F}_{2}$ \\
def2-QZVPP & 0.57 & 0.21 & 0.57 & 0.61 & 1.09 & $79 \mathrm{~F}_{2}$ \\
\hline \hline
\end{tabular}


TABLE II: Statistical measures for the error per valence electron $(\mathrm{kJ} / \mathrm{mol})$ in the Hartree-Fock contributions to the atomisation energies, including the contribution from CABS singles.

\begin{tabular}{|c|c|c|c|c|c|c|}
\hline Basis & $\bar{\Delta}$ & $\sigma$ & $\Delta_{\text {abs }}$ & $\Delta_{\mathrm{rms}}$ & $\Delta_{\max }$ & Molecule \\
\hline & & & 0.54 & 0.56 & 1.47 & 106 \\
\hline cc-pVTZ-F12 & -0.10 & 0.02 & 0.10 & 0.10 & -0.15 & $95 \mathrm{H}_{2} \mathrm{O}$ \\
\hline cc-pVQZ- & -0.01 & 0.00 & 0.01 & 0.01 & -0.02 & $73 \mathrm{FH}$ \\
\hline aug- & -0 & 0.15 & 0.52 & 0.54 & & $106 \mathrm{H}_{2}$ \\
\hline pVTZ & -0.1 & 0.05 & 0.13 & 0.13 & 47 & $106 \mathrm{H}_{2}$ \\
\hline aug-cc-pVQZ & -0.03 & & 03 & 0.03 & -0.13 & $106 \mathrm{H}_{2}$ \\
\hline aug-cc-pV5Z & -0.00 & 0.00 & 0.00 & 0.00 & -0.02 & $106 \mathrm{H}_{2}$ \\
\hline def2-TZVP & -0.15 & 0.07 & 0.15 & 0.17 & -0.51 & $106 \mathrm{H}_{2}$ \\
\hline def2-QZVPP & -0.03 & 0.01 & 0.03 & 0.04 & -0.09 & $98 \mathrm{H}_{3} \mathrm{NO}$ \\
\hline
\end{tabular}




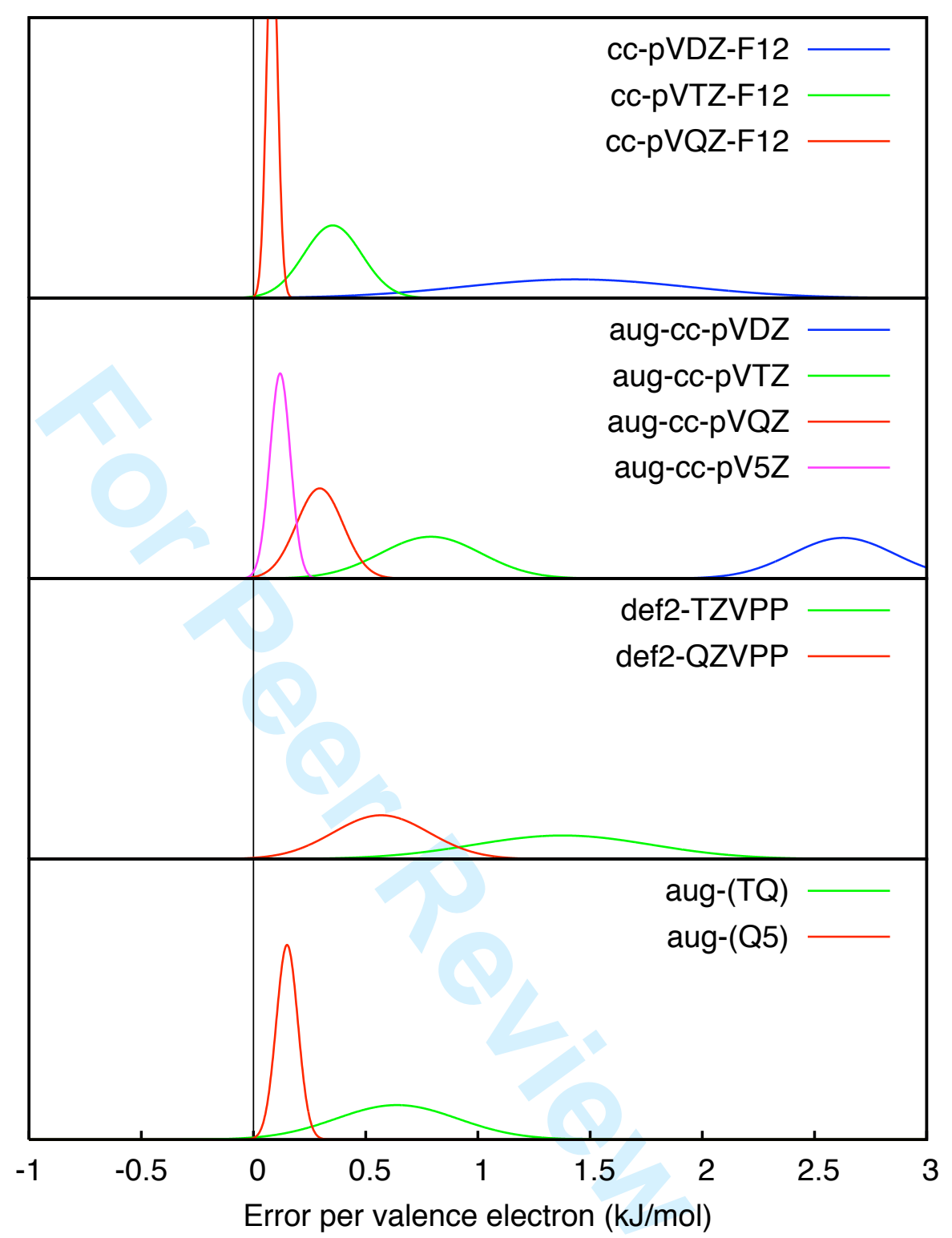

FIG. 1: Performance of basis sets and extrapolation methods for absolute correlation energies (fixed-amplitudes approach). 
FIG. 2: Mean errors per valence electron $(\mathrm{kJ} / \mathrm{mol})$ in the Hartree-Fock contribution, plus CABS singles, to the atomisation energy as a function of the size of the basis. $(\times=\mathrm{DZ}, \bullet=\mathrm{TZ}, \square=$ $\mathrm{QZ}, \diamond=5 \mathrm{Z}$.)

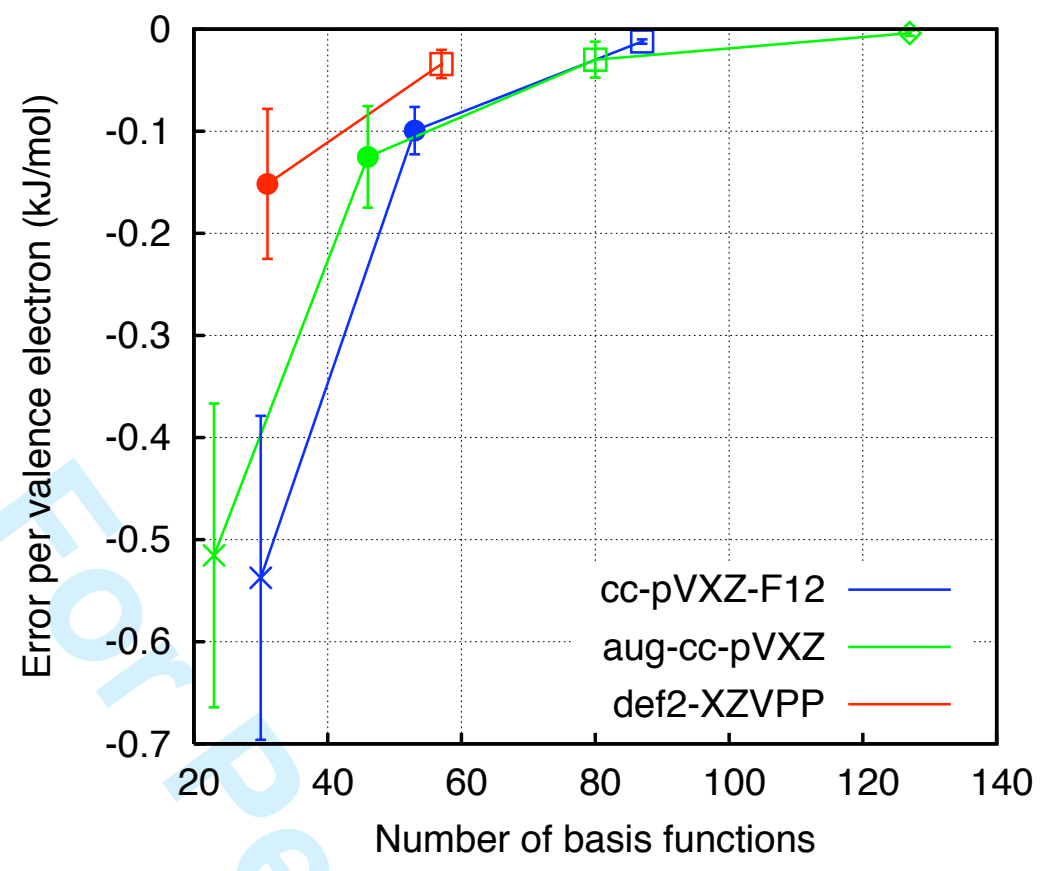


1. CFN
0.00000000000000
0.00000000000000
2.52142953000000
n
0.00000000000000
0.00000000000000
0.32960818000000
c
0.00000000000000
0.00000000000000
$-2.06665065000000$
f

2. CFN

0.00000000000000

0.00000000000000

2.67876778000000

0.44778857000000

0.00000000000000

0.00000000000000

$-2.02204519000000$

0.00000000000000

0.00000000000000

c

$n$

f

3. CF2

$0.00000000000000 \quad 0.00000000000000$

1.13735013000000

$-1.94744090000000$

$-0.35919339000000$

0.00000000000000

1.94744090000000

$-0.35919339000000$

C

$f$

0.00000000000000

$f$

4. CF20

0.00000000000000

0.00000000000000

$-2.52032782000000$

$-0.30282732000000$

0.00000000000000

1.15658025000000

2.00284701000000

0.00000000000000

1.15658025000000

0.00000000000000

5. CF4

0.00000000000000

0.00000000000000

0.00000000000000

1.43694780000000

$-2.03215107000000$

1.43694780000000

0.00000000000000

2.03215107000000

$-1.43694780000000$

0.00000000000000

$-1.43694780000000 \quad f$

0

C

$f$

$f$

$-2.03215107000000$

0.00000000000000

6. CHF

$-1.44124597000000$

0.19321598000000

0.00000000000000

0.00000000000000

0.00000000000000

C

$-1.82961741000000$

$-0.02498408000000$

h

1.02040691000000

f

7. CHFO

$-2.17326766000000$

0.40734985000000

0.00000000000000

0.00000000000000

$-0.77472903000000$

0.00000000000000

0.00000000000000

C

$f$

$f$

$f$

$f$

2.01089662000000

0.29638019000000

o

C

f

$-0.08219885000000$

$-2.82740334000000$

h

8. CHF3

0.00000000000000

$-0.00000007000000$

$-0.68407173000000$

0.19241534000000

0.19241553000000

2.04213199000000

2.35805074000000

0.19241525000000

$-2.04213196000000$

$-1.17902520000000$

$-2.73648082000000$

C

$f$

$-1.17902547000000$

$f$

$-0.00000045000000$

0.00000369000000

9. $\mathrm{CHN}$

0.00000000000000

0.00000000000000

1.12808134000000

0.00000000000000

0.00000000000000

$-1.05819935000000$

0.00000000000000

$-3.07415887000000$

h 
10. CHN
0.00000000000000
0.00000000000000
1.30138538000000
c
0.00000000000000
0.00000000000000
$-0.91401776000000$
0.00000000000000
$-2.79568988000000$
$\mathrm{n}$
0.00000000000000

$-0.01099896000000$

0.00000000000000

0.00000000000000

$-0.01220102000000$

0.00000000000000

0.00000000000000

$-1.57306597000000$

$-2.18811088000000$

$-2.86812939000000$

- -

12. CHNO
2.21163205000000
0.00720438000000
$-2.28391108000000$
$-3.45252629000000$

0.00619865000000

0.00000000000000

0.00000000000000

$-0.07372328000000$

0.00000000000000

0.15272168000000

$-1.34253506000000$

0.00000000000000

n

c

o

h

0.00550485000000

0.00000000000000

0.00000000000000

$-0.04360924000000$

0.00000000000000

0.00000000000000

o

c

$\mathrm{n}$

h

$-2.13593554000000$

0.08482663000000

2.33567860000000

$-0.49146000000000$

--

14. CHNO

$-2.61079650000000$

0.02702212000000

0.00000000000000

c

$-0.38735302000000$

$-0.07083949000000$

0.00000000000000

0.14151153000000

0.00000000000000

$-1.58336974000000$

0.00000000000000

o

n

c

$\mathrm{h}$

15. $\mathrm{CH} 2$
0.00000000000000
0.00000000000000
$-1.62507784000000$
0.00000000000000
1.62507784000000

0.19028088000000

$-1.13282092000000$

$-1.13282092000000$

c

$\mathrm{h}$

$-$

1.04523458000000

0.00000000000000

0.00000000000000

$-0.44589227000000$

0.00000000000000

2.18275032000000

$-1.71351724000000$

2.18275032000000

1.71351724000000

n

o

h

$-$

17. $\mathrm{CH} 2 \mathrm{~N} 2$

$-0.02878484000000$

2.47541237000000

0.00000000000000

0.00000000000000

0.02974710000000

0.27970632000000

0.00000000000000

1.57711797000000

$-3.03504248000000$

$-1.57711797000000$

C

h

18. $\mathrm{CH} 2 \mathrm{~N} 2$

$$
\begin{aligned}
& \mathrm{n} \\
& \mathrm{c} \\
& \mathrm{n} \\
& \mathrm{h} \\
& \mathrm{h}
\end{aligned}
$$


0.00000001000000

$-1.16250776000000$

1.16250775000000

$-0.00000002000000$

$-0.00000002000000$

19. $\mathrm{CH} 2 \mathrm{~N} 2$

0.00000000000000

0.00000000000000

0.00000000000000

$-1.80150104000000$

1.80150104000000

$-$

20. $\mathrm{CH} 20$

0.00000000000000

0.00000000000000

1.76826429000000

$-1.76826429000000$

21. $\mathrm{CH} 20$

1.39296356000000

$-1.06259714000000$

2.14849648000000

$-1.87008721000000$

$--$

22. $\mathrm{CH} 202$

0.00000079000000

$-1.43132506000000$

1.43132415000000

0.00000248000000

0.00000248000000

--

23. $\mathrm{CH} 2 \mathrm{O} 2$

0.18761237000000

$-2.14377916000000$

2.12012349000000

$-1.91471639000000$

0.05628056000000

--

24. $\mathrm{CH} 2 \mathrm{O3}$

3.28740464000000

1.15680145000000

0.48788352000000

$-0.80282208000000$

$-3.13551429000000$

$-3.93096124000000$

$-$

25. CH3F

1.21094997000000

$-1.39533765000000$
0.00000000000000

0.00000000000000

0.00000000000000

$-1.76176832000000$

1.76176832000000

0.00000000000000

0.00000000000000

0.00000000000000

0.00000000000000

0.00000000000000

0.00000000000000

0.00000000000000

0.00000000000000

0.00000000000000

0.23103879000000

$-0.15735766000000$

$-1.73191484000000$

1.47835587000000

1.47894910000000

$-0.71597656000000$

$-0.71597819000000$

2.55828371000000

2.55828371000000

0.78014928000000

$-0.23008725000000$

$-0.40551724000000$

$-2.04403303000000$

2.84243372000000

0.06078038000000

$-0.66751303000000$

$-2.62413899000000$

1.01629436000000

$-0.42654297000000$

0.24769101000000

0.00000000000000

0.00000000000000
$-1.64702632000000$

0.89785518000000

0.89785519000000

$-2.66968431000000$

$-2.66968431000000$

0.14666465000000

2.29958167000000

$-2.30962800000000$

$-3.24432921000000$

$-3.24432921000000$

c

n

n

$\mathrm{h}$

h

$-1.13924972000000$

1.14181204000000

2.24267974000000

2.24267974000000

0.00000000000000

0.00000000000000

0.00000000000000

0.00000000000000

n

$n$

c

h

h

o

c

h

$\mathrm{h}$

0.00000000000000

0.00000000000000

0.00000000000000

$-1.74615726000000$

1.74615726000000

C

o

h

h

$0.00000000000000 \quad$ с

$0.00000000000000 \quad 0$

$0.00000000000000 \quad 0$

$0.00000000000000 \mathrm{~h}$

$0.00000000000000 \quad h$

0.00967029000000

$0.02311423000000 \mathrm{c}$

$0.07063902000000 \quad \mathrm{~h}$

$-0.03513396000000 \quad 0$

$-0.08522933000000 \quad 0$

$1.41092214000000 \quad \mathrm{~h}$

$0.00000000000000 \quad f$

$0.00000000000000 \quad c$ 
$-2.07114788000000$

$-2.07114788000000$

$-2.07114788000000$

$--$

26. $\mathrm{CH} 3 \mathrm{~N}$

1.19406252000000

$-1.20695588000000$

2.31708157000000

2.22977672000000

$-1.99448845000000$

$--$

27. $\mathrm{CH} 3 \mathrm{NO}$

2.14328512000000

0.16850560000000

0.07926462000000

$-2.16534090000000$

$-3.71456457000000$

$-2.30055259000000$

28. $\mathrm{CH} 3 \mathrm{~N} 02$

$-2.49260327000000$

$-1.11459223000000$

1.51225485000000

2.20091391000000

$-4.46324545000000$

$-2.05513323000000$

$-2.05513323000000$

$--$

29. CH3NO2

0.17436670000000

1.22253822000000

1.22274672000000

$-2.64180801000000$

$-3.27540670000000$

$-3.27527266000000$

$-3.22492365000000$

$--$

30. $\mathrm{CH} 4$

0.00000000000000

0.00000000000000

0.00000000000000

1.67804050000000

$-1.67804050000000$

-

31. CH4N20

0.00000000000000

0.00000000000000

0.09441766000000

$-0.09441766000000$

$-0.82696611000000$
0.97171065000000

$-1.94342131000000$

0.97171065000000

0.03367679000000

$-0.15385846000000$

$-1.68634047000000$

1.81706777000000

1.60605227000000

$-0.39191435000000$

0.76574432000000

2.84545735000000

$-0.30943100000000$

0.75083000000000

$-2.19456595000000$

1.02343164000000

$-1.30731974000000$

$-1.00623815000000$

1.12260969000000

0.45981739000000

2.13343254000000

2.13343257000000

0.00000201000000

2.05683357000000

$-2.05672445000000$

$-0.00011723000000$

1.70576415000000

$-1.70678474000000$

0.00065671000000

0.00000000000000

$-1.67804050000000$

1.67804050000000

0.00000000000000

0.00000000000000

0.00000000000000

0.00000000000000

2.18732228000000

$-2.18732228000000$

2.12258736000000
$-1.68305222000000$

0.00000000000000

1.68305222000000

h

h

h

0.00000000000000

0.00000000000000

0.00000000000000

0.00000000000000

0.00000000000000

$-0.00333931000000$

$-0.00443768000000$

0.00374760000000

0.03244580000000

$-0.20940383000000$

$-0.13932104000000$

c

n

h

h

h

$0.00000000000000 \quad \mathrm{c}$

$0.00000000000000 \quad 0$

$0.00000000000000 \mathrm{n}$

$0.00000000000000 \quad 0$

$0.00000000000000 \mathrm{~h}$

$-1.67649002000000 \quad h$

$1.67649004000000 \quad h$

$-0.01693596000000$

0.00506180000000

0.00506027000000

$-0.00132710000000$

$-0.93924454000000$

$-0.93789058000000$

1.96760627000000

0

c

h

n

h

h

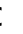

n

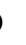

h

$h$

$0.00000000000000 \quad \mathrm{c}$

$1.18655382000000 \quad \mathrm{~h}$

$1.18655382000000 \quad h$

$-1.18655382000000 \quad h$

$-1.18655382000000 \quad h$

$0.18046562000000 \quad \mathrm{c}$

$2.47207620000000 \quad 0$

$-1.26200369000000 \quad \mathrm{n}$

$-1.26200369000000 \quad \mathrm{n}$

$-2.92396516000000 \quad h$ 

$-0.29815295000000$
0.82696611000000
0.29815295000000

$--$

32. $\mathrm{CH} 40$

$-1.30369282000000$

$-1.94098743000000$

1.37260736000000

2.07619542000000

2.10597127000000

2.10597127000000

$--$

33. $\mathrm{CH} 5 \mathrm{~N}$

1.39891351000000

$-1.36585859000000$

2.13355077000000

2.13355040000000

2.16396330000000

$-2.05498519000000$

$-2.05498510000000$

$--$

34. CO

0.00000000000000

0.00000000000000

--

35. $\mathrm{CO2}$
0.00000000000000
0.00000000000000
0.00000000000000

36. C2F2
0.00000000000000
0.00000000000000
0.00000000000000
0.00000000000000

$--$

37. C2F4

$-1.25113369000000$

1.25113369000000

$-2.61445496000000$

$-2.61445496000000$

2.61445496000000

2.61445496000000

$--$

38. $\mathrm{C} 2 \mathrm{HF}$
0.00000000000000
0.00000000000000
0.00000000000000
0.00000000000000

3.73693829000000

$-2.12258736000000$

$-3.73693829000000$

0.12062409000000

$-1.57442367000000$

$-0.02434411000000$

1.90651404000000

$-0.97831056000000$

$-0.97831056000000$

0.00000001000000

0.00000000000000

1.65717331000000

$-1.65717583000000$

0.00000226000000

1.52298081000000

$-1.52298069000000$

0.00000000000000

0.00000000000000

0.00000000000000

0.00000000000000

0.00000000000000

0.00000000000000

$-2.19907016000000$

2.19907016000000

1.22281928000000

$-0.91740604000000$

C

o

0.00000000000000

0.00000000000000

0.00000000000000

0.00000000000000

$-1.12394864000000$

$-3.55529102000000$

1.12394864000000

3.55529102000000

0.00000000000000

0.00000000000000

2.07526472000000

$-2.07526472000000$

$-2.07526472000000$

2.07526472000000

0.00000000000000

0.00000000000000

0.00000000000000

0.00000000000000

0.00000000000000

0.00000000000000

0.00000000000000

0.00000000000000

0.00000000000000

0.00000000000000
$-2.59519804000000$

$-4.60033561000000$

$-0.32792481000000$

2.09037608000000 h

h

h 
39. C2HF3

$$
\begin{array}{r}
-0.79092889000000 \\
1.41564645000000 \\
-1.08977167000000 \\
-2.96645888000000 \\
1.57447455000000 \\
3.57811639000000 \\
--\quad \\
40 . \mathrm{C} 2 \mathrm{H} 2
\end{array}
$$

$$
\begin{array}{r}
-0.10550487000000 \\
-1.29157828000000 \\
2.35448669000000 \\
-1.31138407000000 \\
-3.31856218000000 \\
0.01538254000000
\end{array}
$$

0.00000000000000

0.00000000000000

0.00000000000000

0.00000000000000

0.00000000000000

0.00000000000000

0.00000000000000

0.00000000000000

0.00000000000000

0.00000000000000

0.00000000000000

0.00000000000000

0.00000000000000

0.00000000000000

0.00000000000000

0.00000000000000

0.00000000000000

0.00000000000000

0.00000000000000

0.00000000000000
0.00000000000000

0.00000000000000

0.00000000000000

0.00000000000000

0.00000000000000

0.00000000000000

$-1.14018500000000$

$-3.14954873000000$

1.14018500000000

3.14954873000000

0.27031747000000

2.76726337000000

$-1.15919817000000$

$-1.15919817000000$

3.76794499000000

3.76794499000000
C

C

$f$

$f$

h

$f$

C

h

c

h

$0.03947147000000 \quad \mathrm{c}$

$2.23953625000000 \quad 0$

$-2.44728091000000 \quad \mathrm{c}$

$-3.43684544000000 \quad \mathrm{~h}$

$-3.43684544000000 \quad h$

$-1.61868677000000 \quad 0$

$0.94631837000000 \quad \mathrm{c}$

$0.94631837000000 \quad \mathrm{c}$

$1.57721618000000 \quad \mathrm{~h}$

$1.57721618000000 \quad h$

$0.00000000000000 \quad c$

$0.00000000000000 \quad \mathrm{c}$

$0.00000000000000 \quad 0$

$0.00000000000000 \quad 0$

$0.00000000000000 \quad \mathrm{~h}$

$0.00000000000000 \quad h$

$0.00000000000000 \quad \mathrm{c}$

$0.00000000000000 \quad \mathrm{c}$

$0.00000000000000 \quad \mathrm{f}$

$0.00000000000000 \quad \mathrm{~h}$

$0.00000000000000 \quad h$ 


\begin{tabular}{|c|c|c|c|}
\hline-2.44073255000000 & -2.44998169000000 & 0.00000000000000 & $\mathrm{~h}$ \\
\hline-- & & & \\
\hline \multicolumn{4}{|l|}{ 46. C2H3FO } \\
\hline-2.75966864000000 & 0.02367322000000 & 0.00000000000000 & c \\
\hline 0.04807514000000 & -0.29394736000000 & 0.00000000000000 & c \\
\hline 1.20284480000000 & 1.98569648000000 & 0.00000000000000 & $f$ \\
\hline 1.25506325000000 & -2.18004637000000 & 0.00000000000000 & o \\
\hline-3.66449749000000 & -1.81541413000000 & 0.00000000000000 & $\mathrm{~h}$ \\
\hline-3.32124411000000 & 1.10014267000000 & 1.66126378000000 & $\mathrm{~h}$ \\
\hline-3.32124411000000 & 1.10014267000000 & -1.66126378000000 & $\mathrm{~h}$ \\
\hline \multicolumn{4}{|l|}{-- } \\
\hline \multicolumn{4}{|l|}{ 47. $\mathrm{C} 2 \mathrm{H} 3 \mathrm{~N}$} \\
\hline 2.50650267000000 & 0.00000000000000 & 0.00000000000000 & $\mathrm{n}$ \\
\hline 0.31674268000000 & 0.00000000000000 & 0.00000000000000 & c \\
\hline-2.44866743000000 & 0.00000000000000 & 0.00000000000000 & c \\
\hline-3.14725442000000 & 0.96674518000000 & -1.67445176000000 & $\mathrm{~h}$ \\
\hline-3.14725442000000 & -1.93349035000000 & 0.00000000000000 & $\mathrm{~h}$ \\
\hline-3.14725442000000 & 0.96674518000000 & 1.67445176000000 & $\mathrm{~h}$ \\
\hline \multicolumn{4}{|l|}{--} \\
\hline \multicolumn{4}{|l|}{ 48. $\mathrm{C} 2 \mathrm{H} 3 \mathrm{~N}$} \\
\hline 2.60412057000000 & 0.00000000000000 & 0.00000000000000 & c \\
\hline 0.38773957000000 & 0.00000000000000 & 0.00000000000000 & $\mathrm{n}$ \\
\hline-2.30320556000000 & 0.00000000000000 & 0.00000000000000 & c \\
\hline-2.99011097000000 & 0.96817775000000 & -1.67693305000000 & $\mathrm{~h}$ \\
\hline-2.99011097000000 & -1.93635549000000 & 0.00000000000000 & $\mathrm{~h}$ \\
\hline-2.99011097000000 & 0.96817775000000 & 1.67693305000000 & $\mathrm{~h}$ \\
\hline \multicolumn{4}{|l|}{--} \\
\hline \multicolumn{4}{|l|}{ 49. $\mathrm{C} 2 \mathrm{H} 4$} \\
\hline-1.26036625000000 & 0.00000000000000 & 0.00000000000000 & c \\
\hline 1.26036625000000 & 0.00000000000000 & 0.00000000000000 & c \\
\hline-2.32786582000000 & 1.74395356000000 & 0.00000000000000 & $\mathrm{~h}$ \\
\hline-2.32786582000000 & -1.74395356000000 & 0.00000000000000 & $\mathrm{~h}$ \\
\hline 2.32786582000000 & -1.74395356000000 & 0.00000000000000 & $\mathrm{~h}$ \\
\hline 2.32786582000000 & 1.74395356000000 & 0.00000000000000 & $\mathrm{~h}$ \\
\hline \multicolumn{4}{|l|}{--} \\
\hline \multicolumn{4}{|l|}{$50 . \mathrm{C} 2 \mathrm{H} 40$} \\
\hline-2.37887825000000 & 0.31264719000000 & 0.00000000000000 & c \\
\hline 0.23651287000000 & -0.79399013000000 & 0.00000000000000 & c \\
\hline 2.16055287000000 & 0.43825704000000 & 0.00000000000000 & 0 \\
\hline 0.32314625000000 & -2.88334967000000 & 0.00000000000000 & $\mathrm{~h}$ \\
\hline-3.40825520000000 & -0.35283014000000 & -1.65968527000000 & $\mathrm{~h}$ \\
\hline-3.40825520000000 & -0.35283014000000 & 1.65968527000000 & $\mathrm{~h}$ \\
\hline-2.28740008000000 & 2.36482055000000 & 0.00000000000000 & $\mathrm{~h}$ \\
\hline \multicolumn{4}{|l|}{-- } \\
\hline \multicolumn{4}{|l|}{ 51. $\mathrm{C} 2 \mathrm{H} 40$} \\
\hline 0.00000000000000 & 0.00000000000000 & 1.51389592000000 & 0 \\
\hline 0.00000000000000 & -1.38424374000000 & -0.80423179000000 & $\mathrm{c}$ \\
\hline 0.00000000000000 & 1.38424374000000 & -0.80423179000000 & c \\
\hline 1.73595109000000 & 2.38662116000000 & -1.21873161000000 & $\mathrm{~h}$ \\
\hline-1.73595109000000 & 2.38662116000000 & -1.21873161000000 & $\mathrm{~h}$ \\
\hline
\end{tabular}


$-1.73595109000000$

1.73595109000000

$-$

52. $\mathrm{C} 2 \mathrm{H} 402$

0.07481133000000

1.23550806000000

1.22944673000000

3.02057978000000

$-2.74946140000000$

$-3.61306594000000$

$-3.34076749000000$

$-3.34076749000000$

$-$

53. $\mathrm{C} 2 \mathrm{H} 402$

$-2.56225140000000$

$-1.13518486000000$

1.37159620000000

2.43623603000000

2. 31211795000000

$-2.12594834000000$

$-4.53192008000000$

$-2.12594834000000$

$--$

54. $\mathrm{C} 2 \mathrm{H} 5 \mathrm{~F}$

$-0.05003570000000$

$-2.43452655000000$

$-2.51129958000000$

$-4.08575399000000$

$-2.51126137000000$

0.06771465000000

0.06771348000000

2.04532173000000

$-1$

55. $\mathrm{C} 2 \mathrm{H} 5 \mathrm{~N}$

$-0.81290937000000$

$-0.81290947000000$

1.59129202000000

2.42832528000000

$-1.25758245000000$

$-1.33239116000000$

$-1.33239134000000$

$-1.25758262000000$

$--$

56. $\mathrm{C} 2 \mathrm{H} 6$
0.00000000000000
0.00000000000000
$-0.96042623000000$
$-0.96042623000000$
1.92085245000000
0.96042623000000

$-2.38662116000000$

$-2.38662116000000$

$-1.21873161000000$

$-1.21873161000000$

$\mathrm{h}$

$\mathrm{h}$

$-0.19150106000000$

$-2.14952933000000$

2.09309921000000

1.73668045000000

0.07890881000000

$-1.78008510000000$

1.13980415000000

1.13980415000000

1.03455736000000

$-1.27431544000000$

$-0.93329892000000$

1.07339005000000

$-2.77487012000000$

2.14522423000000

0.46758644000000

2.14522423000000

1.08723679000000

$-0.47827487000000$

$-1.67554803000000$

0.75556316000000

$-1.67558740000000$

2.28139176000000

2.28139171000000

$-0.48899624000000$

$-0.01824980000000$

$-0.01824980000000$

0.15707548000000

$-1.56621527000000$

$-1.77099138000000$

1.68016543000000

1.68016543000000

$-1.77099138000000$

1.44181226000000

$-1.44181226000000$

2.18765019000000

2.18765019000000

2.18765019000000

$-2.18765019000000$
0.00000000000000

0.00000000000000

0.00000000000000

0.00000000000000

0.00000000000000

0.00000000000000

1.66145310000000

$-1.66145310000000$

0.00000000000000

0.00000000000000

0.00000000000000

0.00000000000000

0.00000000000000

$-1.67406739000000$

0.00000000000000

1.67406739000000

$-0.00000021000000$

0.00000031000000

1.67239943000000

$-0.00003207000000$

$-1.67237440000000$

$-1.67589743000000$

1.67590020000000

0.00000016000000
C

o

o

h

c

h

h

h

C

o

c

o

h

h

h

h
$-1.39926949000000$

1.39926943000000

0.00000006000000

0.00000011000000

$-2.35360027000000$

$-2.40733205000000$

2.40733195000000

2.35360018000000

C

c

h

h

h

h

h

f

$0.00000000000000 \quad \mathrm{c}$

$0.00000000000000 \quad \mathrm{c}$

$-1.66350702000000 \quad \mathrm{~h}$

$1.66350702000000 \quad h$

$0.00000000000000 \quad h$

$1.66350702000000 \quad h$ 
$-1.92085245000000$

0.96042623000000

$--$

57. $\mathrm{C} 2 \mathrm{H} 60$

0.00000000000000

0.00000000000000

0.00000000000000

0.00000000000000

1.67978782000000

$-1.67978782000000$

0.00000000000000

$-1.67978782000000$

1.67978782000000

$--$

58. $\mathrm{C} 2 \mathrm{H} 60$

2.37439392000000

$-0.01421033000000$

4.03866316000000

2.43876310000000

2.43876310000000

$-2.09482234000000$

$-3.61886989000000$

$-0.07663518000000$

$-0.07663518000000$

$--$

59. C2N2

0.00000000000000

0.00000000000000

0.00000000000000

0.00000000000000

--

60. $\mathrm{C3H} 3 \mathrm{~N}$

1.30670793000000

3.41520168000000

$-1.30047208000000$

$-3.18485379000000$

$-1.66063192000000$

$-2.81481282000000$

$-5.12930439000000$

$--$

61. $\mathrm{C} 3 \mathrm{H} 4$

0.00000000000000

0.00000000000000

0.00000000000000

$-1.24053110000000$

1.24053110000000

1.24053110000000

$-1.24053110000000$
$-2.18765019000000$

$-2.18765019000000$

0.00000000000000

$-2.19099633000000$

2.19099633000000

3.81531793000000

2.27430378000000

2.27430378000000

$-3.81531793000000$

$-2.27430378000000$

$-2.27430378000000$

0.47460827000000

$-1.09614203000000$

$-0.74106301000000$

1.67283758000000

1.67283758000000

0.61495029000000

$-0.36550049000000$

$-2.29916148000000$

$-2.29916148000000$

0.00000000000000

0.00000000000000

0.00000000000000

0.00000000000000

0.21055444000000

$-0.39937751000000$

0.96474326000000

$-0.72157431000000$

2.97597901000000

$-2.73046020000000$

$-0.09883445000000$

0.00000000000000

0.00000000000000

0.00000000000000

$-1.24053110000000$

1.24053110000000

$-1.24053110000000$

1.24053110000000
0.00000000000000

$-1.66350702000000$

h

h

1.03664117000000

$-0.47392414000000$

$-0.47392414000000$

0.78500641000000

$-1.68409835000000$

$-1.68409835000000$

0.78500641000000

$-1.68409835000000$

$-1.68409835000000$

0.00000000000000

0.00000000000000

0.00000000000000

$-1.67279483000000$

1.67279483000000

0.00000000000000

0.00000000000000

$-1.68157736000000$

1.68157736000000

$-1.31129555000000$

$-3.50678141000000$

1.31129555000000

3.50678141000000

0.00000000000000

0.00000000000000

0.00000000000000

0.00000000000000

0.00000000000000

0.00000000000000

0.00000000000000

0

c

C

h

h

h

h

h

$\mathrm{h}$

C

c

h

h

h

o

h

h

h

C

$\mathrm{n}$

c

n

0.00000000000000

$-2.47604173000000$

2.47604173000000

$-3.52667610000000$

$-3.52667610000000$

3.52667610000000

3.52667610000000 c

n

c

C

$\mathrm{h}$

$\mathrm{h}$

$\mathrm{h}$

62. $\mathrm{C} 3 \mathrm{H} 4$ 
0.00000000000000

1.22593157000000

$-1.22593157000000$

2.98234645000000

$-2.98234645000000$

0.00000000000000

0.00000000000000

63. $\mathrm{C} 3 \mathrm{H} 4$

4.59158401000000

2.58312887000000

0.29998635000000

$-2.46498773000000$

$-3.19005213000000$

$-3.19005213000000$

$-3.19005213000000$ $--$

64. C3H6

$-1.42335627000000$

0.00000000000000

1.42335627000000

$-2.37549751000000$

$-2.37549751000000$

0.00000000000000

0.00000000000000

2.37549751000000

2.37549751000000

$--$

65. $\mathrm{C} 3 \mathrm{H} 6$

0.19859574000000

$-2.37219797000000$

2.36821992000000

0.26289871000000

4.17520504000000

2.38675384000000

$-2.23419330000000$

$-3.45397191000000$

$-3.45397192000000$

66. $\mathrm{C} 3 \mathrm{H} 8$

0.00000000000000

0.00000000000000

0.00000000000000

$-1.65350148000000$

1.65350148000000

0.00000000000000

1.66501856000000

$-1.66501856000000$

0.00000000000000

$-1.66501856000000$
0.00000000000000

0.00000000000000

0.00000000000000

0.00000000000000

0.00000000000000

$-1.72414206000000$

1.72414206000000

0.00000000000000

0.00000000000000

0.00000000000000

0.00000000000000

0.96285137000000

$-1.92570273000000$

0.96285137000000

0.00000000000000

0.00000000000000

0.00000000000000

$-1.71970254000000$

1.71970254000000

$-1.71970254000000$

1.71970254000000

$-1.71970254000000$

1.71970254000000

0.85246489000000

$-0.33728363000000$

$-0.43394360000000$

2.90294665000000

0.52109284000000

$-2.48158114000000$

$-2.39244745000000$

0.24135301000000

0.24135313000000

0.00000000000000

$-2.39154719000000$

2.39154719000000

0.00000005000000

$-0.00000005000000$

4.09283690000000

2.45471345000000

2.45471345000000

$-4.09283690000000$

$-2.45471345000000$
1.67032917000000

$-0.90732372000000$

$-0.90732372000000$

$-1.92526236000000$

$-1.92526236000000$

2.78444885000000

2.78444885000000

0.00000000000000

0.00000000000000

0.00000000000000

0.00000000000000

$-1.66770749000000$

0.00000000000000

1.66770749000000

$-0.82177513000000$

1.64355025000000

$-0.82177513000000$

$-1.37149413000000$

$-1.37149413000000$

2.74298826000000

2.74298826000000

$-1.37149413000000$

$-1.37149413000000$

h

c

C

c

h

h

h

0.00000000000000

0.00000000000000

0.00000000000000

0.00000002000000

0.00000000000000

$-0.00000001000000$

$-0.00000002000000$

1.65910262000000

$-1.65910258000000$

C

C

c

h

h

$\mathrm{h}$

h

h

$\mathrm{h}$

1.09953897000000

$-0.51058937000000$

$-0.51058937000000$

2.33657145000000

2.33657145000000

0.65250387000000

$-1.72779314000000$

$-1.72779314000000$

0.65250387000000

$-1.72779314000000$ c

c

$\mathrm{h}$

h

h h

C

c

c

h

h

h

$\mathrm{h}$

h

$\mathrm{h}$

c

c

c

h

h

h

h

h

h

h

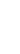

西

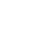




$$
\begin{array}{rr} 
& 1.66501856000000 \\
-- & \\
67 . C 302 & 0.00000000000000 \\
0.00000000000000 & 0.00000000000000 \\
0.00000000000000 & 0.00000000000000 \\
-- & \\
68 . C 4 H 4 \\
1.20074802000000 \\
3.70001456000000 \\
-1.20074802000000 \\
-3.70001456000000 \\
4.75372427000000 \\
4.75372427000000 \\
-4.75372427000000 \\
-4.75372427000000 \\
--\quad \\
69 . C 4 H 4
\end{array}
$$

$-1.48309661000000$

$-1.48309661000000$

1.48309661000000

1.48309661000000

$-2.92295276000000$

2.92295276000000

2.92295282000000

$-2.92295282000000$

70. $\mathrm{C} 4 \mathrm{H} 4$
0.06079470000000
1.00753194000000
0.54382209000000
$-1.61214873000000$
2.19459869000000
1.18454932000000
$-3.51157053000000$
0.13242255000000

--

71. $\mathrm{C} 4 \mathrm{H} 4$

1.28960121000000

$-1.31437183000000$

3.20466685000000

1.67199863000000

5.14180117000000

2.86343407000000

$-3.53285481000000$

$-5.47461652000000$

$--$

72. C4N2
$-2.45471345000000$

$-1.72779314000000$

h

2.36933394000000

$-0.07068827000000$

0.26860305000000

$-0.57467061000000$

$-0.07068827000000$

0.26860305000000

$-2.36933394000000$

$-4.53883956000000$

0.00000000000000

0.00000000000000

0.00000000000000

0.00000000000000

1.75342623000000

$-1.75342623000000$

1.75342623000000

$-1.75342623000000$

1.27007989000000

$-1.27007988000000$

$-1.27007988000000$

1.27007989000000

$-2.71346795000000$

$-2.71346795000000$

2.71346789000000

2.71346789000000

$-0.67225052000000$

$-0.90709986000000$

1.62383880000000

$-0.04448841000000$

$-1.97583827000000$

3.53703374000000

$-0.09690433000000$

$-1.46429116000000$

$-1.57466510000000$

1.04749042000000

$-0.05091199000000$

0.57808667000000

2.28163590000000

$-0.11089617000000$

1.25918414000000

$-3.42992396000000$

0.93524550000000

0.00000000000000

0.00000000000000

0.00000000000000

0.20529117000000

0.00000000000000

2.94629125000000

0.00000000000000

0.00000000000000

0.00000000000000

0.00000000000000
C

o

c

C

o

C

C

C

c

h

h

h

$\mathrm{h}$

C

C

C

C

h

h

h

h
$-2.73758663000000$

$-0.35579767000000$

$-0.87062198000000$

C

C

C

c

h

h

h

h

c

c

h

h

h

c

h 

0.00000000000000
0.00000000000000
0.00000000000000
0.00000000000000
0.00000000000000
0.00000000000000

0.00000000000000

0.00000000000000

0.00000000000000

0.00000000000000

$--$

74. FHO
$-1.28873538000000$

1.42045125000000

1.75022231000000 --

75. $\mathrm{FHO2}$

$-2.09577992000000$

0.16611757000000

2.17177583000000

2.40326102000000

$--$

76. FH2N
$-0.44931251000000$

1.12813525000000

$-0.53021529000000$

$-1.01948108000000$

1.43223958000000

$-1.25496270000000$

1.87856547000000

1.87856547000000

$-0.56756432000000$

1.08870296000000

$-0.23595035000000$

$-0.70599384000000$

1.97799546000000

$-0.43907381000000$

78. FNO
2.18870238000000

0.18962040000000

3.41402056000000

3.01555030000000

$-0.00258348000000$

$-2.09391854000000$
0.26992224000000

$-0.96654909000000$

0.52557748000000
5.94932710000000

3.74891031000000

1.14586252000000

$-1.14586252000000$

$-3.74891031000000$

$-5.94932710000000$ n

c

C

c

c

n

0.08726567000000

$-1.64503588000000$

\section{$f$}

h

$0.00000000000000 \quad f$

$0.00000000000000 \quad 0$

$0.00000000000000 \mathrm{~h}$

$0.00744442000000 \mathrm{f}$

$-0.03062671000000 \quad 0$

$0.12413049000000 \quad 0$

$-1.62430694000000 \quad h$

$0.00000000000000 \quad n$

$0.00000000000000 \quad f$

$1.52224055000000 \quad h$

$-1.52224055000000 \quad h$

$-0.00396791000000 \quad n$

$0.14100350000000 \quad n$

$1.40739625000000 \quad \mathrm{~h}$

$-1.71265419000000 \quad h$

$-1.54867204000000 \quad h$

$-0.00265719000000 \mathrm{f}$

$0.00000000000000 \quad f$

$0.00000000000000 \quad \mathrm{n}$

$0.00000000000000 \quad 0$

$1.33533246000000 \mathrm{f}$

$-1.33533246000000 \mathrm{f}$

$-1.36821970000000 \quad n$

$-1.36821970000000 \quad n$

$1.00846800000000 \quad f$

$1.00846800000000 \quad f$ 
81. F2N2
0.73040438000000
$-0.73040438000000$
3.11075612000000
$-3.11075612000000$

82. F20
0.00000000000000
0.00000000000000
0.00000000000000
$-0.00000048000000$
$-2.08367785000000$
2.08367825000000

$--$

83. F202
$-0.72706080000000$
0.72706080000000
0.20417247000000
$-0.20417247000000$

$--$

0.93071308000000

$-0.93071308000000$

2.79032114000000

$-2.79032114000000$
0.00000000000000

0.00000000000000

0.00000000000000

0.00000000000000

0.00000146000000

$-2.32184071000000$

1.16091982000000

1.16091982000000

0.22568186000000

$--$

85. HNO

$-1.92609525000000$

$-1.15180624000000$

1.12973376000000

86. HNO2

$-1.66852451000000$

$-2.08556680000000$

0.24853238000000

1.97311627000000

$--$

87. HNO2

$-3.26409484000000$

$-2.04284418000000$

0.28007343000000

2.00331585000000

88. HNO2

0.00000000000000

0.00000000000000

0.00000000000000

0.00000000000000

$--$

89. HNO3
1.67417225000000

$-0.16138489000000$

0.03579962000000

2.03675328000000

0.24138638000000

$-0.95842884000000$

0.46935570000000

0.92931401000000

$-0.42567575000000$

0.93720871000000

$-0.45337795000000$

0.00000000000000

0.00000000000000

2.07139629000000

$-2.07139629000000$

0.04815858000000

$-1.61183973000000$
1.16333141000000

$-0.48970933000000$

$-0.48970906000000$

$-1.07157978000000$

$-1.07157978000000$

0.90217198000000

0.90217198000000

0.00000000000000

0.00000000000000

2.01077228000000

$-2.01077228000000$

0.00000000000000

0.00000000000000

0.00000000000000

h

n

o

$0.00000000000000 \quad h$

$0.00000000000000 \quad 0$

$0.00000000000000 \quad \mathrm{n}$

$0.00000000000000 \quad 0$

$0.00000000000000 \quad \mathrm{~h}$

$0.00000000000000 \quad 0$

$0.00000000000000 \quad \mathrm{n}$

$0.00000000000000 \quad 0$

$2.59393531000000 \quad \mathrm{~h}$

$0.64047550000000 \quad \mathrm{n}$

$-0.36207942000000 \quad 0$

$-0.36207942000000 \quad 0$

$0.00000000000000 \quad \mathrm{n}$

$0.00000000000000 \quad 0$ 
0.50703691000000

$-2.27719833000000$

$-3.19947927000000$

$--$

90. HN3

2.27760667000000

0.13838089000000

$-2.20049793000000$

$-2.99408850000000$

$-$

91. H2N2

0.00000000000000

0.00000000000000

0.00000000000000

0.00000000000000

--

92. H2N2

$-1.16571860000000$

1.16571860000000

1.99591319000000

$-1.99591319000000$

$-$

93. $\mathrm{H} 2 \mathrm{~N} 2$

0.00000000000000

0.00000000000000

1.62874302000000

$-1.62874302000000$

$--$

94. H2N2O

$-2.00096946000000$

$-0.15211795000000$

2.03934575000000

3.57496478000000

1.96006859000000

$--$

95. H2O
0.00000000000000
0.00000000000000
0.00000000000000

--

96. $\mathrm{H} 2 \mathrm{O} 2$

$-1.37036083000000$

1.37036083000000

1.79707295000000

$-1.79707295000000$

$--$

97. NH3
1.76229021000000
0.00000002000000
$-0.88114527000000$

2.31879411000000

$-0.79847083000000$

0.78330030000000

0.01719734000000

$-0.07154591000000$

0.16816761000000

$-1.58144161000000$

$-1.17949353000000$

1.17949353000000

1.90324666000000

$-1.90324666000000$

0.19309095000000

$-0.19309095000000$

1.56693957000000

$-1.56693957000000$

0.00000000000000

0.00000000000000

0.00000000000000

0.00000000000000

0.42453884000000

$-0.93424639000000$

0.33876394000000

$-0.68268524000000$

2.21878744000000

0.00000000000000

$-1.42414130000000$

1.42414130000000

$-0.11253552000000$

0.11253552000000

$-1.36193271000000$

1.36193271000000

0.61319792000000

$-0.13239867000000$

0.61319778000000
0.00000000000000

0.00000000000000

0.00000000000000

0

o

h

0.00000000000000

0.00000000000000

0.00000000000000

0.00000000000000

$-0.12195018000000$

$-0.12195018000000$

1.69441846000000

1.69441846000000

n

$\mathrm{n}$

$\mathrm{n}$

$\mathrm{h}$

0.00000000000000

0.00000000000000

0.00000000000000

0.00000000000000

$\mathrm{n}$

n

h

$\mathrm{h}$

$-1.30156612000000$

1.00111590000000

2.08728032000000

2.08728032000000

$\mathrm{n}$

$\mathrm{n}$

h

$\mathrm{h}$

$-0.00494680000000$

$-0.00805147000000$

0.06117260000000

$-0.38061282000000$

$-0.27896147000000$

$\mathrm{n}$

n

$\mathrm{h}$

$\mathrm{h}$

0.12523284000000

o

$-0.99376801000000$

$-0.99376801000000$

$\mathrm{h}$

h

$-0.05793309000000$

$-0.05793309000000$

0.91944022000000

0.91944022000000

o

n

n

h

h

$0.00000000000000 \quad \mathrm{~h}$

$0.00000000000000 \quad \mathrm{n}$

$1.52618823000000 \quad h$ 


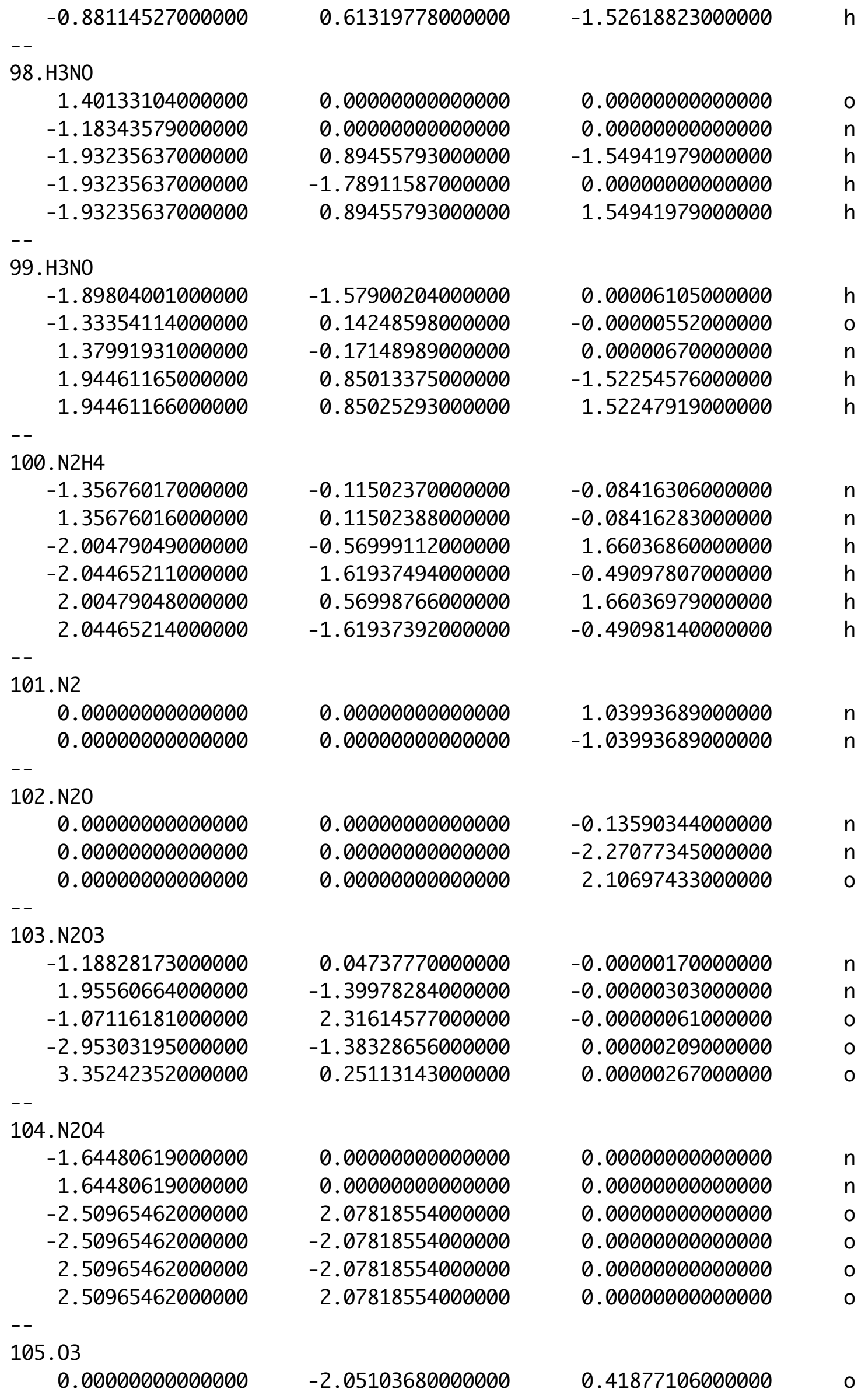


106. H2
0.00000000000000
0.00000000000000
0.70167688000000
h
0.00000000000000
0.00000000000000
$-0.70167688000000$
h 


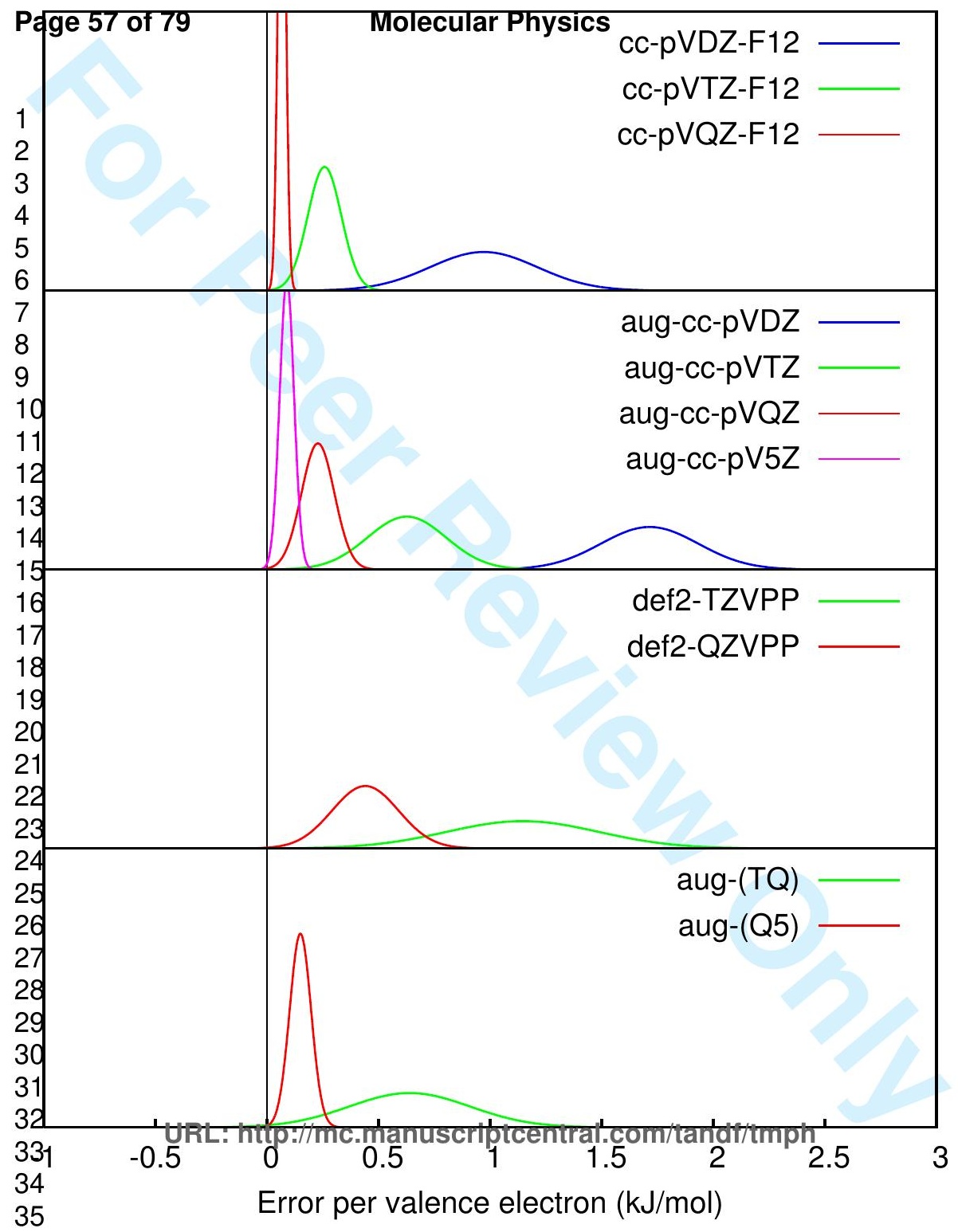




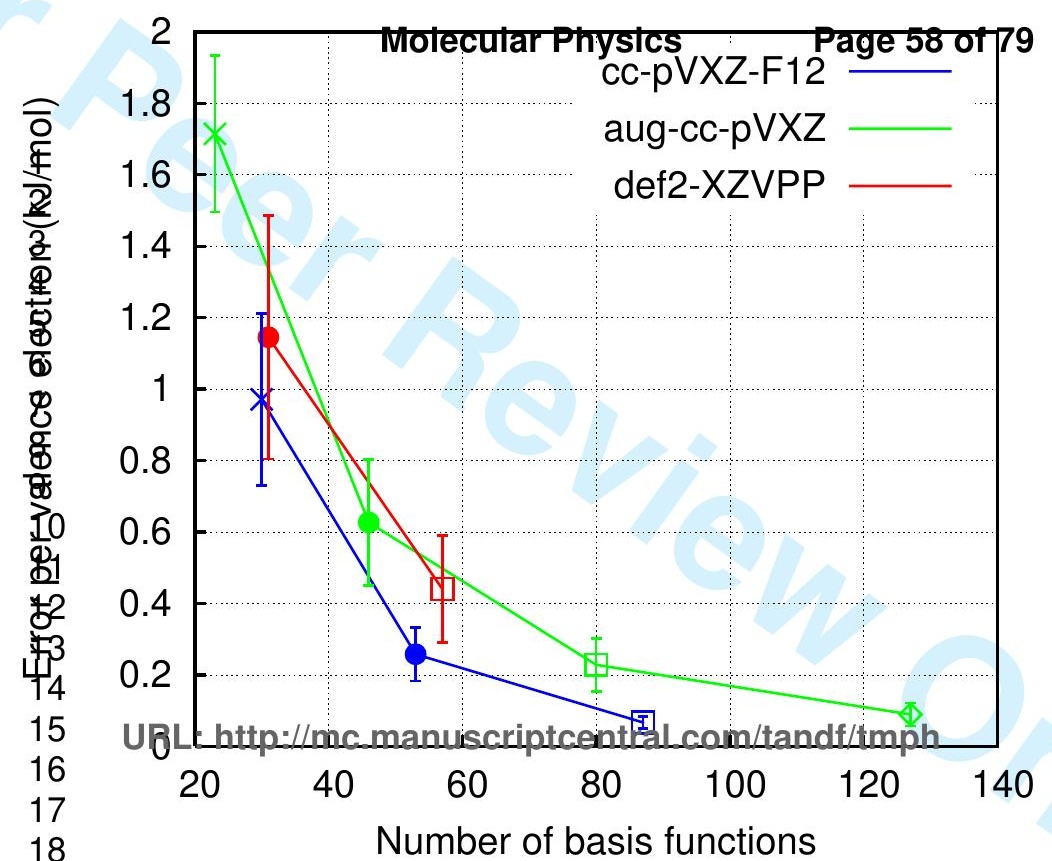




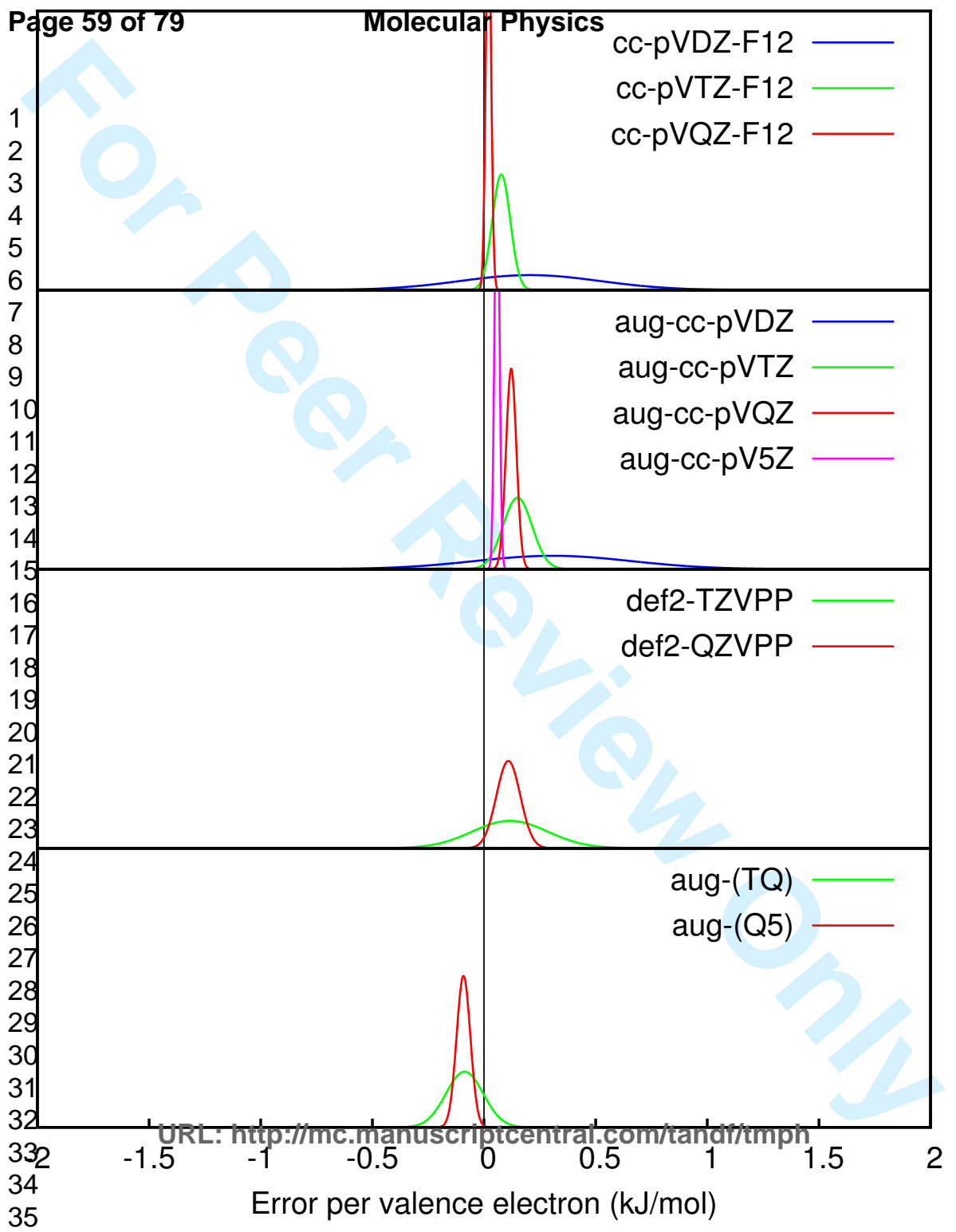




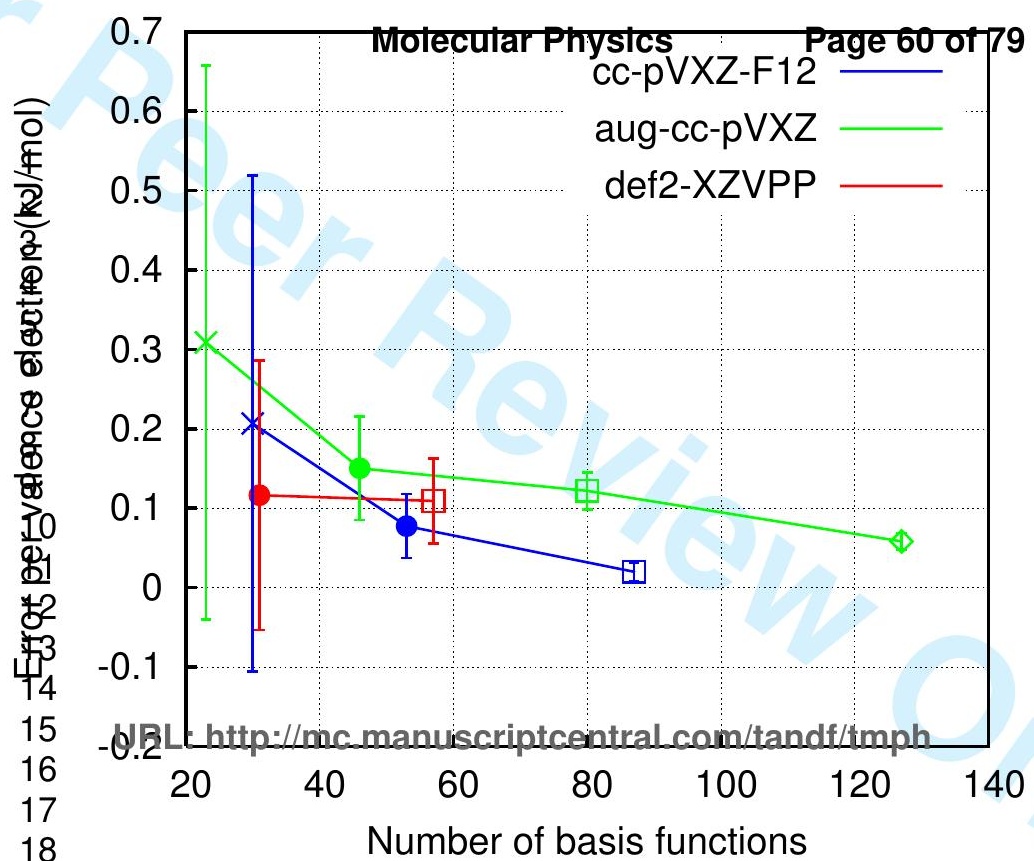




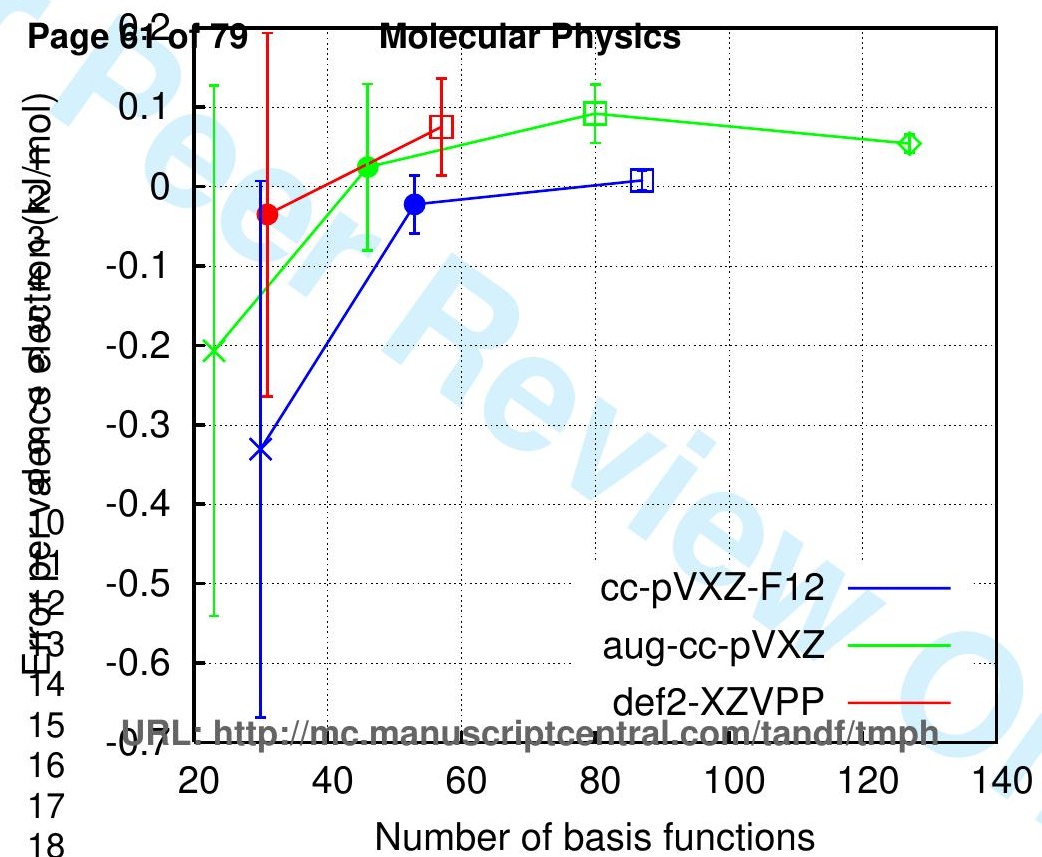




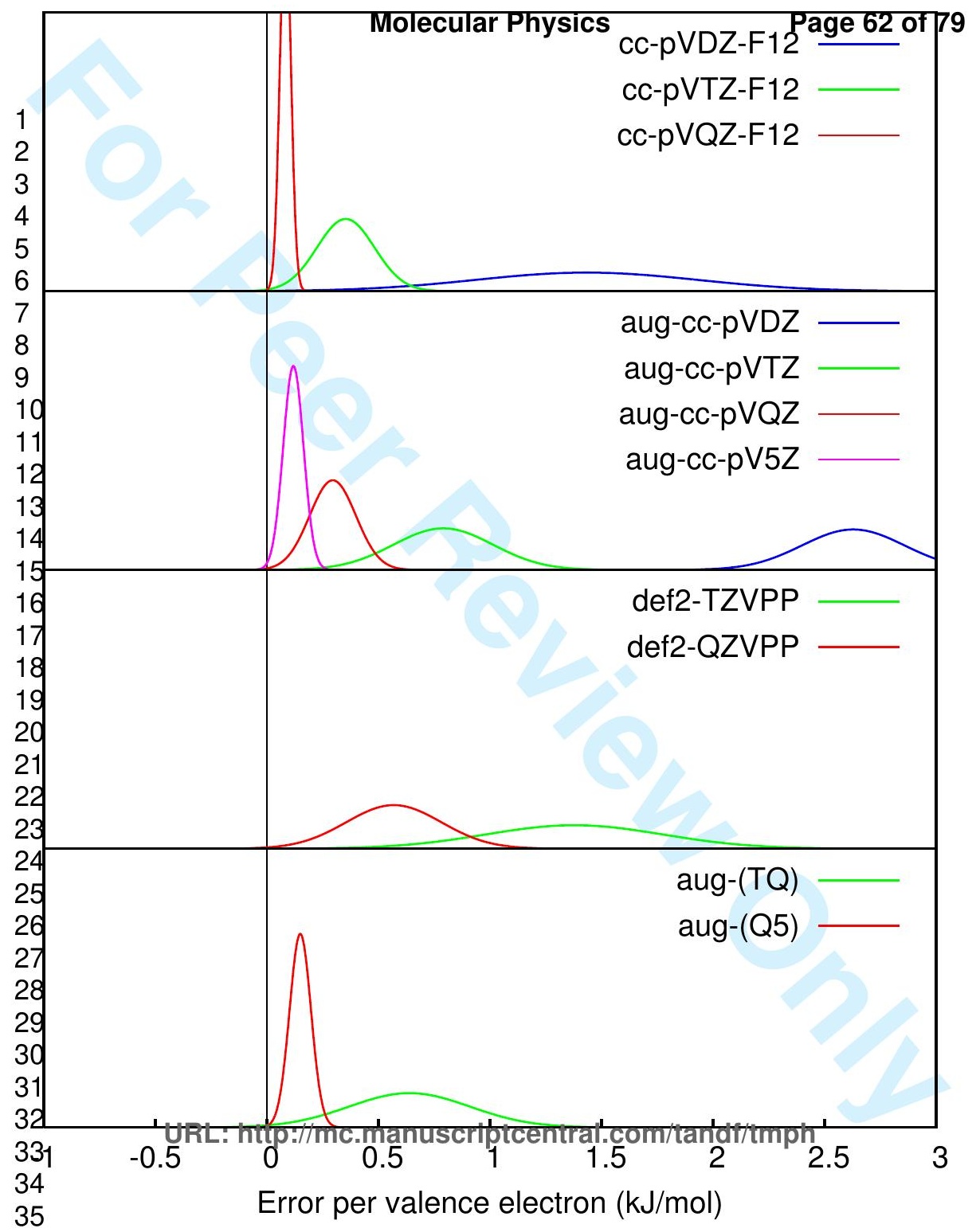




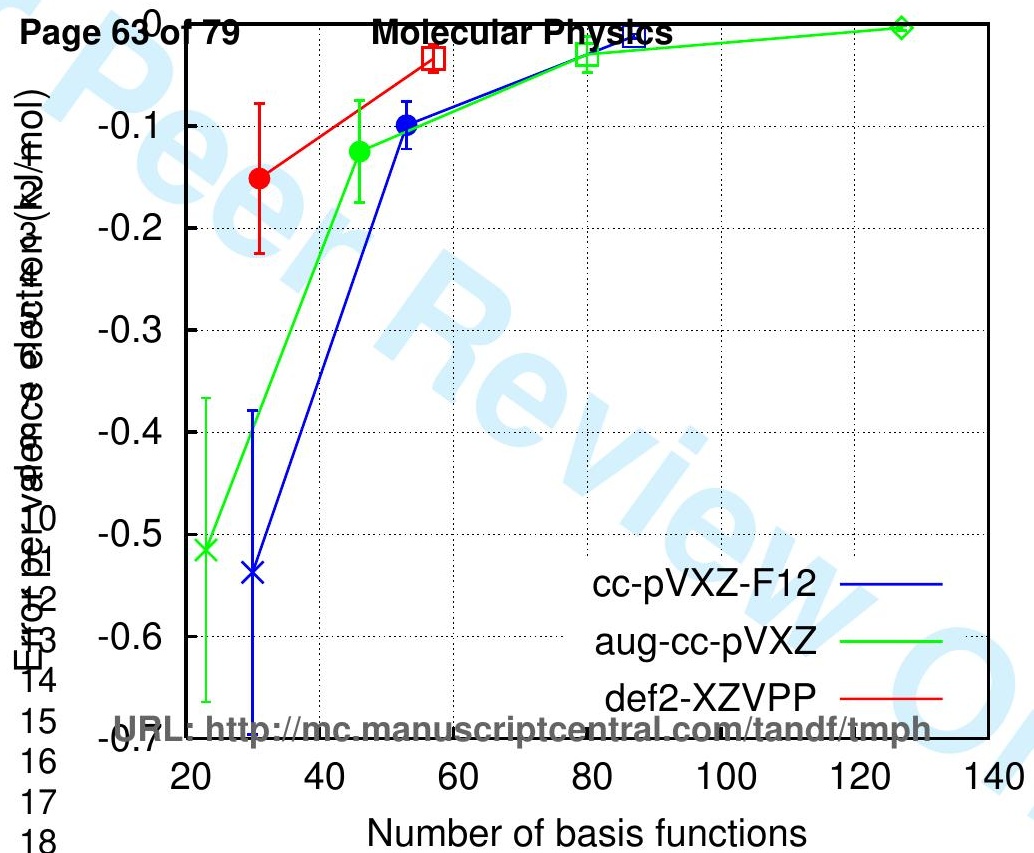


1. CFN
0.00000000000000
0.00000000000000
2.52142953000000
n
0.00000000000000
0.00000000000000
0.32960818000000
c
0.00000000000000
0.00000000000000
$-2.06665065000000$
f

2. CFN

0.00000000000000

0.00000000000000

2.67876778000000

0.44778857000000

0.00000000000000

0.00000000000000

$-2.02204519000000$

0.00000000000000

0.00000000000000

c

$n$

f

3. CF2

$0.00000000000000 \quad 0.00000000000000$

1.13735013000000

$-1.94744090000000$

$-0.35919339000000$

0.00000000000000

1.94744090000000

$-0.35919339000000$

C

$f$

0.00000000000000

$f$

4. CF20

0.00000000000000

0.00000000000000

$-2.52032782000000$

$-0.30282732000000$

0.00000000000000

1.15658025000000

2.00284701000000

0.00000000000000

1.15658025000000

$f$

$f$

5. CF4

0.00000000000000

0.00000000000000

0.00000000000000

1.43694780000000

$-2.03215107000000$

1.43694780000000

0.00000000000000

2.03215107000000

$-1.43694780000000$

0.00000000000000

$-1.43694780000000$

$-2.03215107000000$

0.00000000000000

C

$f$

$f$

$f$

$f$

6. CHF

$-1.44124597000000$

0.19321598000000

0.00000000000000

0.00000000000000

0.00000000000000

c

$-1.82961741000000$

h

1.02040691000000

$-0.02498408000000$

$f$

7. CHFO

$-2.17326766000000$

0.40734985000000

0.00000000000000

0.00000000000000

$-0.77472903000000$

0.00000000000000

2.01089662000000

0.29638019000000

0.00000000000000

o

C

$-2.82740334000000$

f

$-0.08219885000000$

$\mathrm{h}$

8. CHF3

0.00000000000000

$-0.00000007000000$

$-0.68407173000000$

0.19241534000000

0.19241553000000

2.04213199000000

2.35805074000000

0.19241525000000

$-2.04213196000000$

$-1.17902520000000$

$-2.73648082000000$

C

$f$

$f$

$-1.17902547000000$

$f$

0.00000369000000

$-0.00000045000000$

h

9. $\mathrm{CHN}$

0.00000000000000

0.00000000000000

1.12808134000000

0.00000000000000

$-1.05819935000000$

$-3.07415887000000$

0.00000000000000

c

0.00000000000000

h 
10. CHN
0.00000000000000
0.00000000000000
1.30138538000000
c
0.00000000000000
0.00000000000000
$-0.91401776000000$
0.00000000000000
$-2.79568988000000$
$\mathrm{n}$
0.00000000000000

$-0.01099896000000$

0.00000000000000

0.00000000000000

2.47022251000000

$-0.01220102000000$

0.00000000000000

$-2.18811088000000$

0.11790040000000

0.00000000000000

$-1.57306597000000$

$--$

12. CHNO
2.21163205000000
0.00720438000000
$-2.28391108000000$

0.00619865000000

0.00000000000000

0.00000000000000

$-0.07372328000000$

0.15272168000000

$-1.34253506000000$

0.00000000000000

0.00000000000000

n

c

o

h

13. CHNO
$-2.13593554000000$
0.13172988000000
2.33567860000000
4.25802098000000

0.00550485000000

$-0.04360924000000$

0.08482663000000

$-0.49146000000000$

0.00000000000000

0.00000000000000

0.00000000000000

0.00000000000000

o

c

n

h

14. CHNO
$-2.61079650000000$
$-0.38735302000000$
2.12607677000000

0.02702212000000

$-0.07083949000000$

0.00000000000000

0.00000000000000

0.14151153000000

0.00000000000000

$-1.58336974000000$

0.00000000000000

o

n

c

h

15. $\mathrm{CH} 2$
0.00000000000000
0.00000000000000

0.00000000000000

0.19028088000000

$-1.62507784000000$

$-1.13282092000000$

1.62507784000000

$-1.13282092000000$

c

n

o

h

16. $\mathrm{CH} 2 \mathrm{~F} 2$
0.00000001000000
1.04523458000000
$-0.44589227000000$
$-0.44589193000000$
2.07583176000000
2.18275032000000
2.18275032000000
$-0.00000253000000$

0.00000000000000

0.00000000000000

0.00000000000000

$-1.71351724000000$

1.71351724000000

c

h

h

--

2.47541237000000

0.00000000000000

0.00000000000000

0.27970632000000

0.00000000000000

1.57711797000000

$-3.03504248000000$

$-1.57711797000000$

c
$f$
$f$
$h$
$h$

18. $\mathrm{CH} 2 \mathrm{~N} 2$

0.02878484000000

0.09793528000000

$-0.65749699000000$

$-3.03504248000000$
$\mathrm{n}$
$\mathrm{h}$
h

n

c 
0.00000001000000

$-1.16250776000000$

1.16250775000000

$-0.00000002000000$

$-0.00000002000000$

19. $\mathrm{CH} 2 \mathrm{~N} 2$

0.00000000000000

0.00000000000000

0.00000000000000

$-1.80150104000000$

1.80150104000000

$-$

20. $\mathrm{CH} 20$

0.00000000000000

0.00000000000000

1.76826429000000

$-1.76826429000000$

21. $\mathrm{CH} 20$

1.39296356000000

$-1.06259714000000$

2.14849648000000

$-1.87008721000000$

$--$

22. $\mathrm{CH} 202$

0.00000079000000

$-1.43132506000000$

1.43132415000000

0.00000248000000

0.00000248000000

--

23. $\mathrm{CH} 2 \mathrm{O} 2$

0.18761237000000

$-2.14377916000000$

2.12012349000000

$-1.91471639000000$

0.05628056000000

--

24. $\mathrm{CH} 2 \mathrm{O} 3$

3.28740464000000

1.15680145000000

0.48788352000000

$-0.80282208000000$

$-3.13551429000000$

$-3.93096124000000$

--

25. CH3F

1.21094997000000

$-1.39533765000000$
0.00000000000000

0.00000000000000

0.00000000000000

$-1.76176832000000$

1.76176832000000

0.00000000000000

0.00000000000000

0.00000000000000

0.00000000000000

0.00000000000000

0.00000000000000

0.00000000000000

0.00000000000000

0.00000000000000

0.23103879000000

$-0.15735766000000$

$-1.73191484000000$

1.47835587000000

1.47894910000000

$-0.71597656000000$

$-0.71597819000000$

2.55828371000000

2.55828371000000

0.78014928000000

$-0.23008725000000$

$-0.40551724000000$

$-2.04403303000000$

2.84243372000000

0.06078038000000

$-0.66751303000000$

$-2.62413899000000$

1.01629436000000

$-0.42654297000000$

0.24769101000000

0.00000000000000

0.00000000000000
$-1.64702632000000$

0.89785518000000

0.89785519000000

$-2.66968431000000$

$-2.66968431000000$

0.14666465000000

2.29958167000000

$-2.30962800000000$

$-3.24432921000000$

$-3.24432921000000$

c

n

n

$\mathrm{h}$

$\mathrm{h}$

$-1.13924972000000$

1.14181204000000

2.24267974000000

2.24267974000000

0.00000000000000

0.00000000000000

0.00000000000000

0.00000000000000

n

n

c

h

$\mathrm{h}$

o

c

$\mathrm{h}$

$\mathrm{h}$

0.00000000000000

0.00000000000000

0.00000000000000

$-1.74615726000000$

1.74615726000000

C

o

h

$\mathrm{h}$

$0.00000000000000 \quad$ c

$0.00000000000000 \quad 0$

$0.00000000000000 \quad 0$

$0.00000000000000 \mathrm{~h}$

$0.00000000000000 \quad h$

0.00967029000000

$0.02311423000000 \mathrm{c}$

$0.07063902000000 \quad \mathrm{~h}$

$-0.03513396000000 \quad 0$

$-0.08522933000000 \quad 0$

$1.41092214000000 \quad h$

$0.00000000000000 \quad f$

$0.00000000000000 \quad c$ 
$-2.07114788000000$

$-2.07114788000000$

$-2.07114788000000$

$--$

26. $\mathrm{CH} 3 \mathrm{~N}$

1.19406252000000

$-1.20695588000000$

2.31708157000000

2.22977672000000

$-1.99448845000000$

$--$

27. $\mathrm{CH} 3 \mathrm{NO}$

2.14328512000000

0.16850560000000

0.07926462000000

$-2.16534090000000$

$-3.71456457000000$

$-2.30055259000000$

28. $\mathrm{CH} 3 \mathrm{~N} 02$

$-2.49260327000000$

$-1.11459223000000$

1.51225485000000

2.20091391000000

$-4.46324545000000$

$-2.05513323000000$

$-2.05513323000000$

$--$

29. $\mathrm{CH} 3 \mathrm{NO2}$

0.17436670000000

1.22253822000000

1.22274672000000

$-2.64180801000000$

$-3.27540670000000$

$-3.27527266000000$

$-3.22492365000000$

$--$

30. $\mathrm{CH} 4$

0.00000000000000

0.00000000000000

0.00000000000000

1.67804050000000

$-1.67804050000000$

-

31. CH4N20

0.00000000000000

0.00000000000000

0.09441766000000

$-0.09441766000000$

$-0.82696611000000$
0.97171065000000

$-1.94342131000000$

0.97171065000000

0.03367679000000

$-0.15385846000000$

$-1.68634047000000$

1.81706777000000

1.60605227000000

$-0.39191435000000$

0.76574432000000

2.84545735000000

$-0.30943100000000$

0.75083000000000

$-2.19456595000000$

1.02343164000000

$-1.30731974000000$

$-1.00623815000000$

1.12260969000000

0.45981739000000

2.13343254000000

2.13343257000000

0.00000201000000

2.05683357000000

$-2.05672445000000$

$-0.00011723000000$

1.70576415000000

$-1.70678474000000$

0.00065671000000

0.00000000000000

$-1.67804050000000$

1.67804050000000

0.00000000000000

0.00000000000000

0.00000000000000

0.00000000000000

2.18732228000000

$-2.18732228000000$

2.12258736000000
$-1.68305222000000$

0.00000000000000

1.68305222000000

$\mathrm{h}$

h

$\mathrm{h}$

0.00000000000000

0.00000000000000

0.00000000000000

0.00000000000000

0.00000000000000

$-0.00333931000000$

$-0.00443768000000$

0.00374760000000

0.03244580000000

$-0.20940383000000$

$-0.13932104000000$

c

n

h

h

h

$0.00000000000000 \quad$ c

$0.00000000000000 \quad 0$

$0.00000000000000 \quad \mathrm{n}$

$0.00000000000000 \quad \mathrm{o}$

$0.00000000000000 \mathrm{~h}$

$-1.67649002000000 \quad h$

$1.67649004000000 \quad h$

$-0.01693596000000$

0.00506180000000

0.00506027000000

$-0.00132710000000$

$-0.93924454000000$

$-0.93789058000000$

1.96760627000000

0

c

h

n

h

h

$$
\begin{aligned}
& c \\
& n \\
& h \\
& h
\end{aligned}
$$

西

o

o

c

h

$\mathrm{h}$

$\mathrm{h}$

$0.00000000000000 \quad \mathrm{c}$

$1.18655382000000 \quad \mathrm{~h}$

$1.18655382000000 \quad h$

$-1.18655382000000 \quad h$

$-1.18655382000000 \quad h$

$0.18046562000000 \quad \mathrm{C}$

$2.47207620000000 \quad \mathrm{o}$

$-1.26200369000000 \quad \mathrm{n}$

$-1.26200369000000 \quad n$

$-2.92396516000000 \quad h$ 

$-0.29815295000000$
0.82696611000000
0.29815295000000

$--$

32. $\mathrm{CH} 40$

$-1.30369282000000$

$-1.94098743000000$

1.37260736000000

2.07619542000000

2.10597127000000

2.10597127000000

$--$

33. $\mathrm{CH} 5 \mathrm{~N}$

1.39891351000000

$-1.36585859000000$

2.13355077000000

2.13355040000000

2.16396330000000

$-2.05498519000000$

$-2.05498510000000$

$--$

34. CO

0.00000000000000

0.00000000000000

--

35. $\mathrm{CO2}$
0.00000000000000
0.00000000000000
0.00000000000000

36. C2F2
0.00000000000000
0.00000000000000
0.00000000000000
0.00000000000000

$--$

37. C2F4

$-1.25113369000000$

1.25113369000000

$-2.61445496000000$

$-2.61445496000000$

2.61445496000000

2.61445496000000

$--$

38. $\mathrm{C} 2 \mathrm{HF}$
0.00000000000000
0.00000000000000
0.00000000000000
0.00000000000000

3.73693829000000

$-2.12258736000000$

$-3.73693829000000$

0.12062409000000

$-1.57442367000000$

$-0.02434411000000$

1.90651404000000

$-0.97831056000000$

$-0.97831056000000$

0.00000001000000

0.00000000000000

1.65717331000000

$-1.65717583000000$

0.00000226000000

1.52298081000000

$-1.52298069000000$

0.00000000000000

0.00000000000000

0.00000000000000

0.00000000000000

0.00000000000000

0.00000000000000

$-2.19907016000000$

2.19907016000000

1.22281928000000

$-0.91740604000000$

C

o

0.00000000000000

0.00000000000000

0.00000000000000

0.00000000000000

$-1.12394864000000$

$-3.55529102000000$

1.12394864000000

3.55529102000000

0.00000000000000

0.00000000000000

2.07526472000000

$-2.07526472000000$

$-2.07526472000000$

2.07526472000000

0.00000000000000

0.00000000000000

0.00000000000000

0.00000000000000

0.00000000000000

0.00000000000000

0.00000000000000

0.00000000000000

0.00000000000000

0.00000000000000
$-2.59519804000000$

$-4.60033561000000$

$-0.32792481000000$

2.09037608000000 h

h

h 
39. C2HF3

$$
\begin{array}{r}
-0.79092889000000 \\
1.41564645000000 \\
-1.08977167000000 \\
-2.96645888000000 \\
1.57447455000000 \\
3.57811639000000 \\
--\quad \\
40 . \mathrm{C} 2 \mathrm{H} 2
\end{array}
$$

$$
\begin{array}{r}
-0.10550487000000 \\
-1.29157828000000 \\
2.35448669000000 \\
-1.31138407000000 \\
-3.31856218000000 \\
0.01538254000000
\end{array}
$$

0.00000000000000

0.00000000000000

0.00000000000000

0.00000000000000

0.00000000000000

0.00000000000000

0.00000000000000

0.00000000000000

0.00000000000000

0.00000000000000

0.00000000000000

0.00000000000000

0.00000000000000

0.00000000000000

0.00000000000000

0.00000000000000

0.00000000000000

0.00000000000000

0.00000000000000

0.00000000000000
0.00000000000000

0.00000000000000

0.00000000000000

0.00000000000000

0.00000000000000

0.00000000000000

$-1.14018500000000$

$-3.14954873000000$

1.14018500000000

3.14954873000000

0.27031747000000

2.76726337000000

$-1.15919817000000$

$-1.15919817000000$

3.76794499000000

3.76794499000000

0.03947147000000

2.23953625000000

$-2.44728091000000$

$-3.43684544000000$

$-3.43684544000000$

\section{$-1.61868677000000$}

0.94631837000000

0.94631837000000

1.57721618000000

1.57721618000000

C

C

$f$

$f$

h

$f$

c

h

c

h

C

C

$f$

$f$

$\mathrm{h}$

h
C

o

c

h

h

0

C

c

h

h
$0.00000000000000 \quad \mathrm{c}$

$0.00000000000000 \mathrm{C}$

$0.00000000000000 \quad 0$

$0.00000000000000 \quad 0$

$0.00000000000000 \mathrm{~h}$

$0.00000000000000 \mathrm{~h}$

$$
\begin{aligned}
& c \\
& c \\
& 0 \\
& 0 \\
& h
\end{aligned}
$$

0.84714439000000

$-0.41009134000000$

$-0.33252299000000$

2.88063956000000

0.63378204000000
0.00000000000000

0.00000000000000

0.00000000000000

0.00000000000000

0.00000000000000

C

C

$f$

h

h 


\begin{tabular}{|c|c|c|c|}
\hline-2.44073255000000 & -2.44998169000000 & 0.00000000000000 & $\mathrm{~h}$ \\
\hline-- & & & \\
\hline \multicolumn{4}{|l|}{ 46. C2H3FO } \\
\hline-2.75966864000000 & 0.02367322000000 & 0.00000000000000 & c \\
\hline 0.04807514000000 & -0.29394736000000 & 0.00000000000000 & c \\
\hline 1.20284480000000 & 1.98569648000000 & 0.00000000000000 & $f$ \\
\hline 1.25506325000000 & -2.18004637000000 & 0.00000000000000 & o \\
\hline-3.66449749000000 & -1.81541413000000 & 0.00000000000000 & $\mathrm{~h}$ \\
\hline-3.32124411000000 & 1.10014267000000 & 1.66126378000000 & $\mathrm{~h}$ \\
\hline-3.32124411000000 & 1.10014267000000 & -1.66126378000000 & $\mathrm{~h}$ \\
\hline \multicolumn{4}{|l|}{-- } \\
\hline \multicolumn{4}{|l|}{ 47. $\mathrm{C} 2 \mathrm{H} 3 \mathrm{~N}$} \\
\hline 2.50650267000000 & 0.00000000000000 & 0.00000000000000 & $\mathrm{n}$ \\
\hline 0.31674268000000 & 0.00000000000000 & 0.00000000000000 & c \\
\hline-2.44866743000000 & 0.00000000000000 & 0.00000000000000 & c \\
\hline-3.14725442000000 & 0.96674518000000 & -1.67445176000000 & $\mathrm{~h}$ \\
\hline-3.14725442000000 & -1.93349035000000 & 0.00000000000000 & $\mathrm{~h}$ \\
\hline-3.14725442000000 & 0.96674518000000 & 1.67445176000000 & $\mathrm{~h}$ \\
\hline \multicolumn{4}{|l|}{--} \\
\hline \multicolumn{4}{|l|}{ 48. $\mathrm{C} 2 \mathrm{H} 3 \mathrm{~N}$} \\
\hline 2.60412057000000 & 0.00000000000000 & 0.00000000000000 & c \\
\hline 0.38773957000000 & 0.00000000000000 & 0.00000000000000 & $\mathrm{n}$ \\
\hline-2.30320556000000 & 0.00000000000000 & 0.00000000000000 & c \\
\hline-2.99011097000000 & 0.96817775000000 & -1.67693305000000 & $\mathrm{~h}$ \\
\hline-2.99011097000000 & -1.93635549000000 & 0.00000000000000 & $\mathrm{~h}$ \\
\hline-2.99011097000000 & 0.96817775000000 & 1.67693305000000 & $\mathrm{~h}$ \\
\hline \multicolumn{4}{|l|}{--} \\
\hline \multicolumn{4}{|l|}{ 49. $\mathrm{C} 2 \mathrm{H} 4$} \\
\hline-1.26036625000000 & 0.00000000000000 & 0.00000000000000 & c \\
\hline 1.26036625000000 & 0.00000000000000 & 0.00000000000000 & c \\
\hline-2.32786582000000 & 1.74395356000000 & 0.00000000000000 & $\mathrm{~h}$ \\
\hline-2.32786582000000 & -1.74395356000000 & 0.00000000000000 & $\mathrm{~h}$ \\
\hline 2.32786582000000 & -1.74395356000000 & 0.00000000000000 & $\mathrm{~h}$ \\
\hline 2.32786582000000 & 1.74395356000000 & 0.00000000000000 & $\mathrm{~h}$ \\
\hline \multicolumn{4}{|l|}{--} \\
\hline \multicolumn{4}{|l|}{$50 . \mathrm{C} 2 \mathrm{H} 40$} \\
\hline-2.37887825000000 & 0.31264719000000 & 0.00000000000000 & c \\
\hline 0.23651287000000 & -0.79399013000000 & 0.00000000000000 & c \\
\hline 2.16055287000000 & 0.43825704000000 & 0.00000000000000 & 0 \\
\hline 0.32314625000000 & -2.88334967000000 & 0.00000000000000 & $\mathrm{~h}$ \\
\hline-3.40825520000000 & -0.35283014000000 & -1.65968527000000 & $\mathrm{~h}$ \\
\hline-3.40825520000000 & -0.35283014000000 & 1.65968527000000 & $\mathrm{~h}$ \\
\hline-2.28740008000000 & 2.36482055000000 & 0.00000000000000 & $\mathrm{~h}$ \\
\hline \multicolumn{4}{|l|}{-- } \\
\hline \multicolumn{4}{|l|}{ 51. $\mathrm{C} 2 \mathrm{H} 40$} \\
\hline 0.00000000000000 & 0.00000000000000 & 1.51389592000000 & 0 \\
\hline 0.00000000000000 & -1.38424374000000 & -0.80423179000000 & $\mathrm{c}$ \\
\hline 0.00000000000000 & 1.38424374000000 & -0.80423179000000 & c \\
\hline 1.73595109000000 & 2.38662116000000 & -1.21873161000000 & $\mathrm{~h}$ \\
\hline-1.73595109000000 & 2.38662116000000 & -1.21873161000000 & $\mathrm{~h}$ \\
\hline
\end{tabular}


$-1.73595109000000$

1.73595109000000

$-$

52. $\mathrm{C} 2 \mathrm{H} 402$

0.07481133000000

1.23550806000000

1.22944673000000

3.02057978000000

$-2.74946140000000$

$-3.61306594000000$

$-3.34076749000000$

$-3.34076749000000$

$-$

53. $\mathrm{C} 2 \mathrm{H} 402$

$-2.56225140000000$

$-1.13518486000000$

1.37159620000000

2.43623603000000

2. 31211795000000

$-2.12594834000000$

$-4.53192008000000$

$-2.12594834000000$

$--$

54. $\mathrm{C} 2 \mathrm{H} 5 \mathrm{~F}$

$-0.05003570000000$

$-2.43452655000000$

$-2.51129958000000$

$-4.08575399000000$

$-2.51126137000000$

0.06771465000000

0.06771348000000

2.04532173000000

$-1$

55. $\mathrm{C} 2 \mathrm{H} 5 \mathrm{~N}$

$-0.81290937000000$

$-0.81290947000000$

1.59129202000000

2.42832528000000

$-1.25758245000000$

$-1.33239116000000$

$-1.33239134000000$

$-1.25758262000000$

$--$

56. $\mathrm{C} 2 \mathrm{H} 6$
0.00000000000000
0.00000000000000
$-0.96042623000000$
$-0.96042623000000$
1.92085245000000
0.96042623000000

$-2.38662116000000$

$-2.38662116000000$

$-0.19150106000000$

$-2.14952933000000$

2.09309921000000

1.73668045000000

0.07890881000000

$-1.78008510000000$

1.13980415000000

1.13980415000000

1.03455736000000

$-1.27431544000000$

$-0.93329892000000$

1.07339005000000

$-2.77487012000000$

2.14522423000000

0.46758644000000

2.14522423000000

1.08723679000000

$-0.47827487000000$

$-1.67554803000000$

0.75556316000000

$-1.67558740000000$

2.28139176000000

2.28139171000000

$-0.48899624000000$

$-0.01824980000000$

$-0.01824980000000$

0.15707548000000

$-1.56621527000000$

$-1.77099138000000$

1.68016543000000

1.68016543000000

$-1.77099138000000$

1.44181226000000

$-1.44181226000000$

2.18765019000000

2.18765019000000

2.18765019000000

$-2.18765019000000$
$-1.21873161000000$

$-1.21873161000000$

h

h

0.00000000000000

0.00000000000000

0.00000000000000

0.00000000000000

0.00000000000000

0.00000000000000

1.66145310000000

$-1.66145310000000$

0.00000000000000

0.00000000000000

0.00000000000000

0.00000000000000

0.00000000000000

$-1.67406739000000$

0.00000000000000

1.67406739000000

$-0.00000021000000$

0.00000031000000

1.67239943000000

$-0.00003207000000$

$-1.67237440000000$

$-1.67589743000000$

1.67590020000000

0.00000016000000
C

0

o

h

c

h

h

h

C

o

c

o

h

h

h

h
C

c

h

h

h

h

h

$f$

$-1.39926949000000$

1.39926943000000

0.00000006000000

0.00000011000000

$-2.35360027000000$

$-2.40733205000000$

2.40733195000000

2.35360018000000

$0.00000000000000 \quad \mathrm{c}$

$0.00000000000000 \quad \mathrm{c}$

$-1.66350702000000 \quad \mathrm{~h}$

$1.66350702000000 \quad h$

$0.00000000000000 \quad h$

$1.66350702000000 \quad h$ 
$-1.92085245000000$

0.96042623000000

$--$

57. $\mathrm{C} 2 \mathrm{H} 60$

0.00000000000000

0.00000000000000

0.00000000000000

0.00000000000000

1.67978782000000

$-1.67978782000000$

0.00000000000000

$-1.67978782000000$

1.67978782000000

$--$

58. $\mathrm{C} 2 \mathrm{H} 60$

2.37439392000000

$-0.01421033000000$

4.03866316000000

2.43876310000000

2.43876310000000

$-2.09482234000000$

$-3.61886989000000$

$-0.07663518000000$

$-0.07663518000000$

$--$

59. C2N2

0.00000000000000

0.00000000000000

0.00000000000000

0.00000000000000

--

60. $\mathrm{C3H} 3 \mathrm{~N}$

1.30670793000000

3.41520168000000

$-1.30047208000000$

$-3.18485379000000$

$-1.66063192000000$

$-2.81481282000000$

$-5.12930439000000$

$--$

61. $\mathrm{C} 3 \mathrm{H} 4$

0.00000000000000

0.00000000000000

0.00000000000000

$-1.24053110000000$

1.24053110000000

1.24053110000000

$-1.24053110000000$
$-2.18765019000000$

$-2.18765019000000$

0.00000000000000

$-2.19099633000000$

2.19099633000000

3.81531793000000

2.27430378000000

2.27430378000000

$-3.81531793000000$

$-2.27430378000000$

$-2.27430378000000$

0.47460827000000

$-1.09614203000000$

$-0.74106301000000$

1.67283758000000

1.67283758000000

0.61495029000000

$-0.36550049000000$

$-2.29916148000000$

$-2.29916148000000$

0.00000000000000

0.00000000000000

0.00000000000000

0.00000000000000

0.21055444000000

$-0.39937751000000$

0.96474326000000

$-0.72157431000000$

2.97597901000000

$-2.73046020000000$

$-0.09883445000000$

0.00000000000000

0.00000000000000

0.00000000000000

$-1.24053110000000$

1.24053110000000

$-1.24053110000000$

1.24053110000000
0.00000000000000

$-1.66350702000000$

h

h

1.03664117000000

$-0.47392414000000$

$-0.47392414000000$

0.78500641000000

$-1.68409835000000$

$-1.68409835000000$

0.78500641000000

$-1.68409835000000$

$-1.68409835000000$

0.00000000000000

0.00000000000000

0.00000000000000

$-1.67279483000000$

1.67279483000000

0.00000000000000

0.00000000000000

$-1.68157736000000$

1.68157736000000

$-1.31129555000000$

$-3.50678141000000$

1.31129555000000

3.50678141000000

0.00000000000000

0.00000000000000

0.00000000000000

0.00000000000000

0.00000000000000

0.00000000000000

0.00000000000000

0

c

c

h

h

h

h

h

$\mathrm{h}$

C

c

h

h

h

o

h

h

h

C

$\mathrm{n}$

c

n

0.00000000000000

$-2.47604173000000$

2.47604173000000

$-3.52667610000000$

$-3.52667610000000$

3.52667610000000

3.52667610000000 c

n

c

C

$\mathrm{h}$

$\mathrm{h}$

$\mathrm{h}$

62. $\mathrm{C} 3 \mathrm{H} 4$ 
0.00000000000000

1.22593157000000

$-1.22593157000000$

2.98234645000000

$-2.98234645000000$

0.00000000000000

0.00000000000000

63. $\mathrm{C} 3 \mathrm{H} 4$

4.59158401000000

2.58312887000000

0.29998635000000

$-2.46498773000000$

$-3.19005213000000$

$-3.19005213000000$

$-3.19005213000000$

64. C3H6

$-1.42335627000000$

0.00000000000000

1.42335627000000

$-2.37549751000000$

$-2.37549751000000$

0.00000000000000

0.00000000000000

2.37549751000000

2.37549751000000

65. $\mathrm{C} 3 \mathrm{H} 6$

0.19859574000000

$-2.37219797000000$

2.36821992000000

0.26289871000000

4.17520504000000

2.38675384000000

$-2.23419330000000$

$-3.45397191000000$

$-3.45397192000000$

66. $\mathrm{C} 3 \mathrm{H} 8$
0.00000000000000
0.00000000000000
0.00000000000000
$-1.65350148000000$
1.65350148000000
0.00000000000000
1.66501856000000
$-1.66501856000000$
0.00000000000000
$-1.66501856000000$

0.00000000000000

0.00000000000000

0.00000000000000

0.00000000000000

0.00000000000000

$-1.72414206000000$

1.72414206000000

0.00000000000000

0.00000000000000

0.00000000000000

0.00000000000000

0.96285137000000

$-1.92570273000000$

0.96285137000000

0.00000000000000

0.00000000000000

0.00000000000000

$-1.71970254000000$

1.71970254000000

$-1.71970254000000$

1.71970254000000

$-1.71970254000000$

1.71970254000000

0.85246489000000

$-0.33728363000000$

$-0.43394360000000$

2.90294665000000

0.52109284000000

$-2.48158114000000$

$-2.39244745000000$

0.24135301000000

0.24135313000000

0.00000000000000

$-2.39154719000000$

2.39154719000000

0.00000005000000

$-0.00000005000000$

4.09283690000000

2.45471345000000

2.45471345000000

$-4.09283690000000$

$-2.45471345000000$
1.67032917000000

$-0.90732372000000$

$-0.90732372000000$

$-1.92526236000000$

$-1.92526236000000$

2.78444885000000

2.78444885000000

0.00000000000000

0.00000000000000

0.00000000000000

0.00000000000000

$-1.66770749000000$

0.00000000000000

1.66770749000000

$-0.82177513000000$

1.64355025000000

$-0.82177513000000$

$-1.37149413000000$

$-1.37149413000000$

2.74298826000000

2.74298826000000

$-1.37149413000000$

$-1.37149413000000$

0.00000000000000

0.00000000000000

0.00000000000000

0.00000002000000

0.00000000000000

$-0.00000001000000$

$-0.00000002000000$

1.65910262000000

$-1.65910258000000$

1.09953897000000

$-0.51058937000000$

$-0.51058937000000$

2.33657145000000

2.33657145000000

0.65250387000000

$-1.72779314000000$

$-1.72779314000000$

0.65250387000000

$-1.72779314000000$
C

C

c

h

h

h

$\mathrm{h}$

h

C

C

C

h

h

h

C

C

C

h

h

h

h

h

h

C

C

C

h

h

h

h

h

h 


$$
\begin{array}{rr} 
& 1.66501856000000 \\
-- & \\
67 . C 302 & 0.00000000000000 \\
0.00000000000000 & 0.00000000000000 \\
0.00000000000000 & 0.00000000000000 \\
-- & \\
68 . C 4 H 4 \\
1.20074802000000 \\
3.70001456000000 \\
-1.20074802000000 \\
-3.70001456000000 \\
4.75372427000000 \\
4.75372427000000 \\
-4.75372427000000 \\
-4.75372427000000 \\
--\quad \\
69 . C 4 H 4
\end{array}
$$

$-1.48309661000000$

$-1.48309661000000$

1.48309661000000

1.48309661000000

$-2.92295276000000$

2.92295276000000

2.92295282000000

$-2.92295282000000$

70. $\mathrm{C} 4 \mathrm{H} 4$
0.06079470000000
1.00753194000000
0.54382209000000
$-1.61214873000000$
2.19459869000000
1.18454932000000
$-3.51157053000000$
0.13242255000000

$-$

71. $\mathrm{C} 4 \mathrm{H} 4$

1.28960121000000

$-1.31437183000000$

3.20466685000000

1.67199863000000

5.14180117000000

2.86343407000000

$-3.53285481000000$

$-5.47461652000000$

$--$

72. C4N2
$-2.45471345000000$

$-1.72779314000000$

h

2.36933394000000

$-0.07068827000000$

0.26860305000000

$-0.57467061000000$

$-0.07068827000000$

0.26860305000000

$-2.36933394000000$

$-4.53883956000000$

0.00000000000000

0.00000000000000

0.00000000000000

0.00000000000000

1.75342623000000

$-1.75342623000000$

1.75342623000000

$-1.75342623000000$

1.27007989000000

$-1.27007988000000$

$-1.27007988000000$

1.27007989000000

$-2.71346795000000$

$-2.71346795000000$

2.71346789000000

2.71346789000000

$-0.67225052000000$

$-0.90709986000000$

1.62383880000000

$-0.04448841000000$

$-1.97583827000000$

3.53703374000000

$-0.09690433000000$

$-1.46429116000000$

$-1.57466510000000$

1.04749042000000

$-0.05091199000000$

0.57808667000000

2.28163590000000

$-0.11089617000000$

1.25918414000000

$-3.42992396000000$

0.93524550000000

0.00000000000000

0.00000000000000

0.00000000000000

0.20529117000000

0.00000000000000

2.94629125000000

0.00000000000000

0.00000000000000

0.00000000000000

0.00000000000000
C

o

c

C

o

C

C

C

c

h

h

h

$\mathrm{h}$

C

C

C

C

h

h

h

h
$-2.73758663000000$

$-0.35579767000000$

$-0.87062198000000$

C

C

C

c

h

h

h

h

c

c

h

h

h

c

h 

0.00000000000000
0.00000000000000
0.00000000000000
0.00000000000000
0.00000000000000
0.00000000000000

0.00000000000000

0.00000000000000

0.00000000000000

0.00000000000000

$--$

74. $\mathrm{FHO}$
$-1.28873538000000$

1.42045125000000

1.75022231000000 --

75. $\mathrm{FHO2}$

$-2.09577992000000$

0.16611757000000

2.17177583000000

2.40326102000000

$--$

76. FH2N
$-0.44931251000000$

1.12813525000000

$-0.53021529000000$

$-1.01948108000000$

1.43223958000000

$-1.25496270000000$

1.87856547000000

1.87856547000000

$-0.56756432000000$

1.08870296000000

$-0.23595035000000$

$-0.70599384000000$

1.97799546000000

$-0.43907381000000$

78. FNO
2.18870238000000

0.18962040000000

3.41402056000000

3.01555030000000

$-0.00258348000000$

$-2.09391854000000$
0.26992224000000

$-0.96654909000000$

0.52557748000000
5.94932710000000

3.74891031000000

1.14586252000000

$-1.14586252000000$

$-3.74891031000000$

$-5.94932710000000$ n

c

C

c

c

n

0.08726567000000

$-1.64503588000000$

\section{$f$}

h

$0.00000000000000 \quad f$

$0.00000000000000 \quad 0$

$0.00000000000000 \mathrm{~h}$

$0.00744442000000 \mathrm{f}$

$-0.03062671000000 \quad 0$

$0.12413049000000 \quad 0$

$-1.62430694000000 \quad h$

$0.00000000000000 \quad n$

$0.00000000000000 \quad f$

$1.52224055000000 \quad h$

$-1.52224055000000 \quad h$

$-0.00396791000000 \quad n$

$0.14100350000000 \quad n$

$1.40739625000000 \quad \mathrm{~h}$

$-1.71265419000000 \quad h$

$-1.54867204000000 \quad h$

$-0.00265719000000 \mathrm{f}$

$0.00000000000000 \quad f$

$0.00000000000000 \quad \mathrm{n}$

$0.00000000000000 \quad 0$

$1.33533246000000 \mathrm{f}$

$-1.33533246000000 \mathrm{f}$

$-1.36821970000000 \quad n$

$-1.36821970000000 \quad n$

$1.00846800000000 \quad f$

$1.00846800000000 \quad f$ 
81. F2N2
0.73040438000000
$-0.73040438000000$
3.11075612000000
$-3.11075612000000$

82. F20
0.00000000000000
0.00000000000000
0.00000000000000
$-0.00000048000000$
$-2.08367785000000$
2.08367825000000

$--$

83. F202

$-0.72706080000000$

0.72706080000000

0.20417247000000

$-0.20417247000000$

$--$

0.93071308000000

$-0.93071308000000$

2.79032114000000

$-2.79032114000000$
0.00000000000000

0.00000000000000

0.00000000000000

0.00000000000000

0.00000146000000

$-2.32184071000000$

1.16091982000000

1.16091982000000

0.22568186000000

$--$

85. HNO

$-1.92609525000000$

$-1.15180624000000$

1.12973376000000

86. HNO2

$-1.66852451000000$

$-2.08556680000000$

0.24853238000000

1.97311627000000

$--$

87. HNO2

$-3.26409484000000$

$-2.04284418000000$

0.28007343000000

2.00331585000000

88. HNO2

0.00000000000000

0.00000000000000

0.00000000000000

0.00000000000000

$--$

89. HNO3
0.23538296000000
1.76568690000000

1.67417225000000

$-0.16138489000000$

0.03579962000000

2.03675328000000

0.24138638000000

$-0.95842884000000$

0.46935570000000

0.92931401000000

$-0.42567575000000$

0.93720871000000

$-0.45337795000000$

0.00000000000000

0.00000000000000

2.07139629000000

$-2.07139629000000$

0.04815858000000

$-1.61183973000000$
1.16333141000000

$-0.48970933000000$

$-0.48970906000000$

$-1.07157978000000$

$-1.07157978000000$

0.90217198000000

0.90217198000000

0.00000000000000

0.00000000000000

2.01077228000000

$-2.01077228000000$

0.00000000000000

0.00000000000000

0.00000000000000

h

n

o

$0.00000000000000 \quad \mathrm{~h}$

$0.00000000000000 \quad 0$

$0.00000000000000 \quad \mathrm{n}$

$0.00000000000000 \quad 0$

$0.00000000000000 \quad \mathrm{~h}$

$0.00000000000000 \quad 0$

$0.00000000000000 \quad \mathrm{n}$

$0.00000000000000 \quad 0$

$2.59393531000000 \quad \mathrm{~h}$

$0.64047550000000 \quad \mathrm{n}$

$-0.36207942000000 \quad 0$

$-0.36207942000000 \quad 0$

$0.00000000000000 \quad \mathrm{n}$

$0.00000000000000 \quad 0$ 
0.50703691000000

$-2.27719833000000$

$-3.19947927000000$

$--$

90. HN3

2.27760667000000

0.13838089000000

$-2.20049793000000$

$-2.99408850000000$

$-$

91. H2N2

0.00000000000000

0.00000000000000

0.00000000000000

0.00000000000000

--

92. H2N2

$-1.16571860000000$

1.16571860000000

1.99591319000000

$-1.99591319000000$

$-$

93. $\mathrm{H} 2 \mathrm{~N} 2$

0.00000000000000

0.00000000000000

1.62874302000000

$-1.62874302000000$

$--$

94. H2N2O

$-2.00096946000000$

$-0.15211795000000$

2.03934575000000

3.57496478000000

1.96006859000000

$--$

95. H2O
0.00000000000000
0.00000000000000
0.00000000000000

--

96. $\mathrm{H} 2 \mathrm{O} 2$

$-1.37036083000000$

1.37036083000000

1.79707295000000

$-1.79707295000000$

$--$

97. NH3
1.76229021000000
0.00000002000000
$-0.88114527000000$

2.31879411000000

$-0.79847083000000$

0.78330030000000

0.01719734000000

$-0.07154591000000$

0.16816761000000

$-1.58144161000000$

$-1.17949353000000$

1.17949353000000

1.90324666000000

$-1.90324666000000$

0.19309095000000

$-0.19309095000000$

1.56693957000000

$-1.56693957000000$

0.00000000000000

0.00000000000000

0.00000000000000

0.00000000000000

0.42453884000000

$-0.93424639000000$

0.33876394000000

$-0.68268524000000$

2.21878744000000

0.00000000000000

$-1.42414130000000$

1.42414130000000

$-0.11253552000000$

0.11253552000000

$-1.36193271000000$

1.36193271000000

0.61319792000000

$-0.13239867000000$

0.61319778000000
0.00000000000000

0.00000000000000

0.00000000000000

0

o

h

0.00000000000000

0.00000000000000

0.00000000000000

0.00000000000000

$-0.12195018000000$

$-0.12195018000000$

1.69441846000000

1.69441846000000

n

$\mathrm{n}$

$\mathrm{n}$

h

0.00000000000000

0.00000000000000

0.00000000000000

0.00000000000000

$\mathrm{n}$

n

h

h

$-1.30156612000000$

1.00111590000000

2.08728032000000

2.08728032000000

n

n

h

h

$-0.00494680000000$

$-0.00805147000000$

0.06117260000000

$-0.38061282000000$

$-0.27896147000000$

n

n

h

$\mathrm{h}$

0.12523284000000

o

$-0.99376801000000$

$-0.99376801000000$

$\mathrm{h}$

h

$-0.05793309000000$

$-0.05793309000000$

0.91944022000000

0.91944022000000

o

n

n

h

h

$0.00000000000000 \quad h$

$0.00000000000000 \quad \mathrm{n}$

$1.52618823000000 \quad h$ 


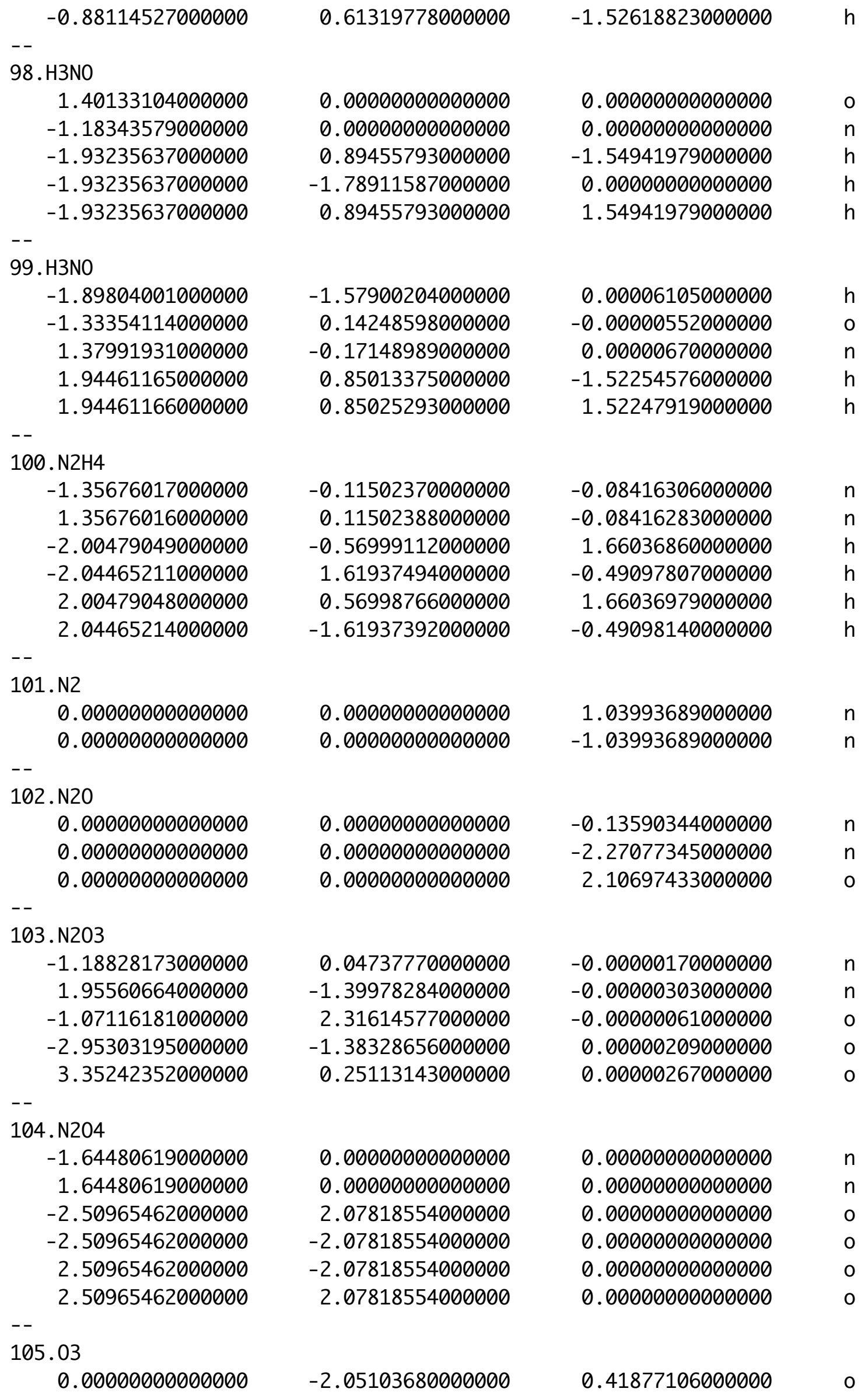


106. H2
0.00000000000000
0.00000000000000
0.70167688000000
h
0.00000000000000
0.00000000000000
$-0.70167688000000$
h 